\title{
Machine-Intelligence-Driven High- Throughput Prediction of 2D Charge Density Wave Phases
}

\author{
Arnab Kabiraj and Santanu Mahapatra* \\ Nano-Scale Device Research Laboratory, Department of Electronic Systems Engineering, \\ Indian Institute of Science (IISc) Bangalore, Bangalore - 560012, India.
}

\section{Supporting Information}


Table S1: All CDW materials and their phases, energy differences with the normal phase, and band/CDW-gaps, as found by our computational methodology. Note the convention here. The normal phase is called $\phi_{0}$, the CDW phase just less stable than $\phi_{0}$ is called $\phi_{-1}$, and the phase just less stable than $\phi_{-1}$ is named $\phi_{-2}$, and so on. Two numbers are in parenthesis beside the $\phi$ names. The first value denotes the energy difference of that CDW phase with its corresponding normal phase in units of meV/formula unit. The second value states the band/CDW-gap of that CDW phase in units of $\mathrm{eV}$. The CDW-gaps are marked with an asterisk (blue font) while the proper band gaps (red font) are not. For example, the notation $\phi_{-2}\left(-26.52,0.5^{*}\right)$ for $\sqrt{ } 13 \times \sqrt{13}$ periodicities of $\mathrm{TaS}_{2}$ - $\mathrm{T}$ means that this is the $2^{\text {nd }} \mathrm{CDW}$ phase found in this periodicity by descending order (lower energy is more stable), the phase is more stable than the normal phase by $26.52 \mathrm{meV} /$ formula-unit and it has a $0.5 \mathrm{eV}$ CDW-gap.

\begin{tabular}{|c|c|c|c|c|}
\hline Material & $2 \times 2$ & $3 \times 3$ & $\sqrt{13} \times \sqrt{13}$ & $4 \times 4$ \\
\hline $\mathrm{TaS}_{2}-\mathrm{T}$ & No phase & $\phi_{-1}(-12,0)$ & $\begin{array}{c}\phi_{-1}(-13.96,0) \\
\phi_{-2}\left(-26.52,0.5^{*}\right)\end{array}$ & $\phi_{-1}(-17.61,0)$ \\
\hline $\mathrm{TaS}_{2}-\mathrm{H}$ & No phase & $\phi_{-1}(-3.92,0)$ & $\begin{array}{c}\phi_{-1}(-0.22,0) \\
\phi_{-2}(-0.7,0)\end{array}$ & $\begin{array}{l}\phi_{-1}(-0.42,0) \\
\phi_{-2}(-1.16,0)\end{array}$ \\
\hline $\mathrm{Hf}_{3} \mathrm{Te}_{2}$ & $\phi_{-1}(-2.26,0)$ & $\phi_{-2}(-2.43,0)$ & NA & $\begin{array}{l}\phi_{-1}(-0.66,0) \\
\phi_{-2}(-2.72,0)\end{array}$ \\
\hline $\mathrm{LaGeI}$ & $\phi_{-1}(-1.62,0)$ & No phase & No phase & $\phi_{-1}(-2.66,0)$ \\
\hline $\mathrm{LaI}_{2}$ & No phase & $\phi_{-1}(-0.64,0)$ & NA & No phase \\
\hline $\mathrm{NbS}_{2}-\mathrm{T}$ & No phase & $\phi_{-1}(-9.6,0)$ & $\phi-1\left(-38.56,0.48^{*}\right)$ & $\phi_{-1}(-14.11,0)$ \\
\hline $\mathrm{NbS}_{2}-\mathrm{H}$ & $\begin{array}{l}\phi_{-1}(-0.43,0) \\
\phi_{-2}(-1.05,0) \\
\phi_{-3}(-2.77,0)\end{array}$ & $\begin{array}{l}\phi_{-1}(-0.71,0) \\
\phi_{-1}(-0.79,0)\end{array}$ & No phase & $\phi_{-1}(-3.9,0)$ \\
\hline $\mathrm{NbSe}_{2}-\mathrm{T}$ & No phase & $\phi-1(-36.21,0)$ & $\phi-1(-68.85,0)$ & $\phi_{-1}(-33.54,0)$ \\
\hline $\mathrm{NbSe}_{2}-\mathrm{H}$ & $\begin{array}{l}\phi_{-1}(-0.22,0) \\
\phi_{-2}(-1.05,0)\end{array}$ & $\begin{array}{c}\phi_{-1}(-2.180) \\
\phi_{-2}(-2.69,0) \\
\phi_{-2}(-2.9,0)\end{array}$ & $\phi_{-1}(-3.31,0)$ & $\begin{array}{c}\phi_{-1}(-1.78,0) \\
\phi_{-2}(-3.59,0) \\
\phi_{-3}(-4.18,0)\end{array}$ \\
\hline $\mathrm{NbTe}_{2}-\mathrm{T}$ & No phase & $\phi-1(-87.24,0)$ & $\phi-1(-93.44,0)$ & $\phi-1(-93.2,0)$ \\
\hline
\end{tabular}




\begin{tabular}{|c|c|c|c|c|}
\hline & & $\phi-2(-111.52,0)$ & & \\
\hline $\mathrm{NbTe}_{2}-\mathrm{H}$ & $\phi_{-1}(-2.02,0)$ & $\phi_{-1}(-16.81,0)$ & $\begin{array}{l}\phi_{-1}\left(-1.08,0.16^{*}\right) \\
\phi_{-2}\left(-4.85,0.25^{*}\right)\end{array}$ & $\phi_{-1}(-25.08,0)$ \\
\hline $\mathrm{OsOCl}_{2}$ & $\phi_{-1}(-0.58,0)$ & No phase & NA & No phase \\
\hline $\mathrm{RuOCl}_{2}$ & $\phi_{-1}(-0.13,0)$ & No phase & NA & No phase \\
\hline $\mathrm{TaSe}_{2}-\mathrm{T}$ & No phase & $\phi_{-1}(-43.18,0)$ & $\phi_{-1}\left(-67.38,0.57^{*}\right)$ & $\phi_{-1}(-34.95,0)$ \\
\hline $\mathrm{TaSe}_{2}-\mathrm{H}$ & $\begin{array}{l}\phi_{-1}(-0.83,0) \\
\phi-2(-1.93,0)\end{array}$ & $\begin{array}{l}\phi_{-1}(-7.16,0) \\
\phi_{-2}(-8.35,0) \\
\phi_{-3}(-8.75,0)\end{array}$ & $\phi_{-1}(-5.72,0)$ & $\phi_{-1}(-3.2,0)$ \\
\hline $\mathrm{TaTe}_{2}-\mathrm{T}$ & No phase & $\phi_{-1}(-128.98,0)$ & $\phi-1(-5.44,0)$ & $\phi_{-1}(-120.72,0)$ \\
\hline $\mathrm{TaTe}_{2}-\mathrm{H}$ & $\begin{array}{c}\phi_{-1}(-6.37,0) \\
\phi_{-2}(-11.97,0)\end{array}$ & $\begin{array}{c}\phi_{-1}(-18.8,0) \\
\phi_{-2}(-23.46,0)\end{array}$ & No phase & $\phi-1(-22.82,0)$ \\
\hline $\mathrm{Ti}_{2} \mathrm{PTe}_{2}$ & $\begin{array}{l}\phi_{-1}(-1.14,0) \\
\phi_{-1}(-1.31,0)\end{array}$ & No phase & No phase & No phase \\
\hline $\mathrm{TiBr}_{2}$ & $\phi_{-1}(-351.36,0.34)$ & $\begin{array}{c}\phi_{-1}(-178.64,0) \\
\phi-2(-284.93,0.41)\end{array}$ & $\phi-1(-286.9,0.18)$ & $\begin{array}{c}\phi-1(-353.97,0.34) \\
\quad \text { repeat of } 2 \times 2\end{array}$ \\
\hline $\mathrm{TiCl}_{2}$ & $\phi_{-1}(-321.45,0.38)$ & $\begin{array}{c}\phi_{-1}(-120.01,0) \\
\phi_{-2}(-216.19,0.44)\end{array}$ & $\phi-1(-232.63,0.15)$ & $\begin{array}{c}\phi_{-1}(-324.810 .38) \\
\text { repeat of } 2 \times 2\end{array}$ \\
\hline $\mathrm{TiOBr}$ & $\begin{array}{c}\phi_{-1}(-5.59,0) \\
\phi_{-2}(-10.86,0)\end{array}$ & $\phi_{-1}(-12.84,0)$ & NA & $\phi_{-1}(-11.78,0)$ \\
\hline $\mathrm{TiOCl}$ & $\begin{array}{c}\phi_{-1}(-7.31,0) \\
\phi_{-2}(-13.37,0)\end{array}$ & $\phi_{-1}(-13.6,0)$ & NA & $\phi_{-1}(-20.33,0.09)$ \\
\hline $\mathrm{TiS}_{2}$ & $\phi-1(-5.76,0.25)$ & $\phi_{-1}(-2.54,0.15)$ & No phase & $\begin{array}{c}\phi-1(-4.64,0.25) \\
\text { repeat of } 2 \times 2\end{array}$ \\
\hline $\mathrm{TiSe}_{2}$ & $\phi_{-1}(-4.55,0)$ & $\phi_{-1}(-0.11,0)$ & No phase & $\phi_{-1}(-6.73,0)$ \\
\hline $\mathrm{TiTe}_{2}$ & $\phi_{-1}(-0.93,0)$ & No phase & No phase & $\phi_{-1}(-0.33,0)$ \\
\hline $\mathrm{VOCl}$ & $\begin{array}{c}\phi_{-1}(-105.63,0) \\
\phi_{-2}(-154.420 .14)\end{array}$ & $\phi_{-1}(-145.87,0)$ & NA & $\begin{array}{c}\phi-1(-140.91,0.14) \\
\text { repeat of } 2 \times 2\end{array}$ \\
\hline $\mathrm{ZrTe}_{2}$ & $\begin{array}{l}\phi_{-1}(-0.16,0) \\
\phi_{-2}(-0.82,0)\end{array}$ & $\phi_{-1}(-0.53,0)$ & $\phi_{-1}(-0.41,0)$ & $\phi_{-1}(-0.27,0)$ \\
\hline
\end{tabular}




\begin{tabular}{|c|c|c|c|c|}
\hline $\mathrm{ZrTe}_{3}$ & $\phi_{-1}(-0.77,0)$ & $\phi_{-1}(-1.1,0)$ & NA & $\phi_{-1}(-0.27,0)$ \\
& $\phi_{-2}(-1.39,0)$ & & & \\
\hline ZrTiSe $_{4}$ & $\phi_{-1}(-4,0.25)$ & $\phi_{-1}(-0.14,0)$ & NA & $\phi-1(-4.43,0.25)$ \\
& & $\phi_{-2}(-0.58,0)$ & & repeat of $2 \times 2$ \\
\hline ZrTiTe $_{4}$ & $\phi_{-1}(-4.29,0)$ & $\phi_{-1}(-0.47,0)$ & NA & $\phi-1(-4.82,0)$ \\
\hline
\end{tabular}

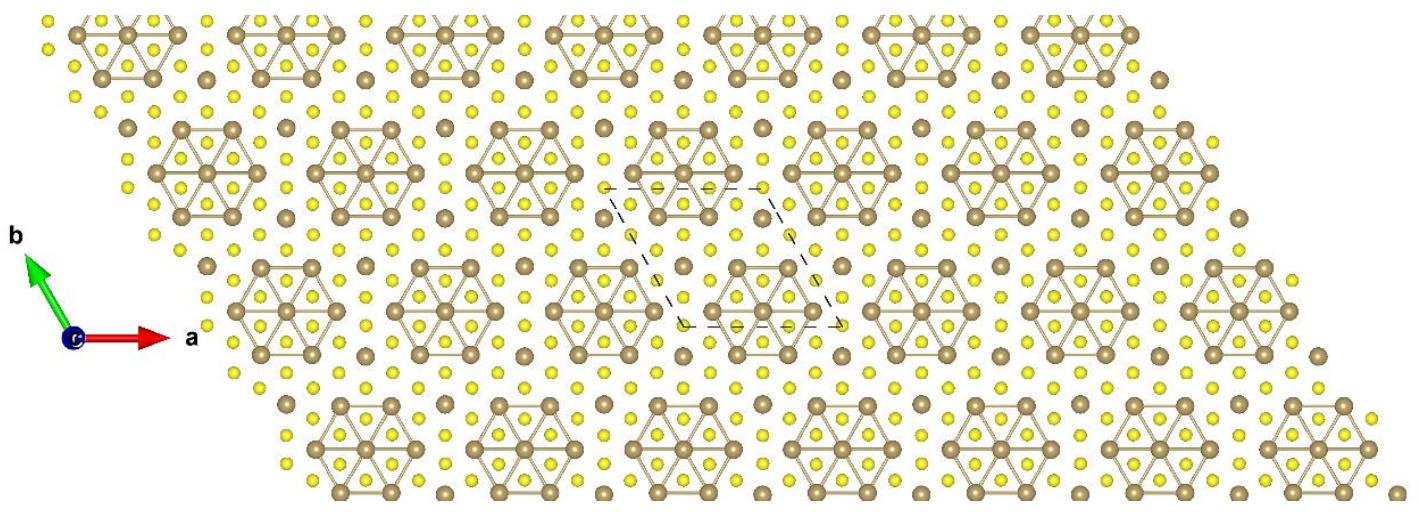

Figure S1: Top view of $\mathrm{TaS}_{2}-\mathrm{T} 3 \times 3 \phi-1$. The brown and yellow balls represent the Ta and $\mathrm{S}$ atoms, respectively.

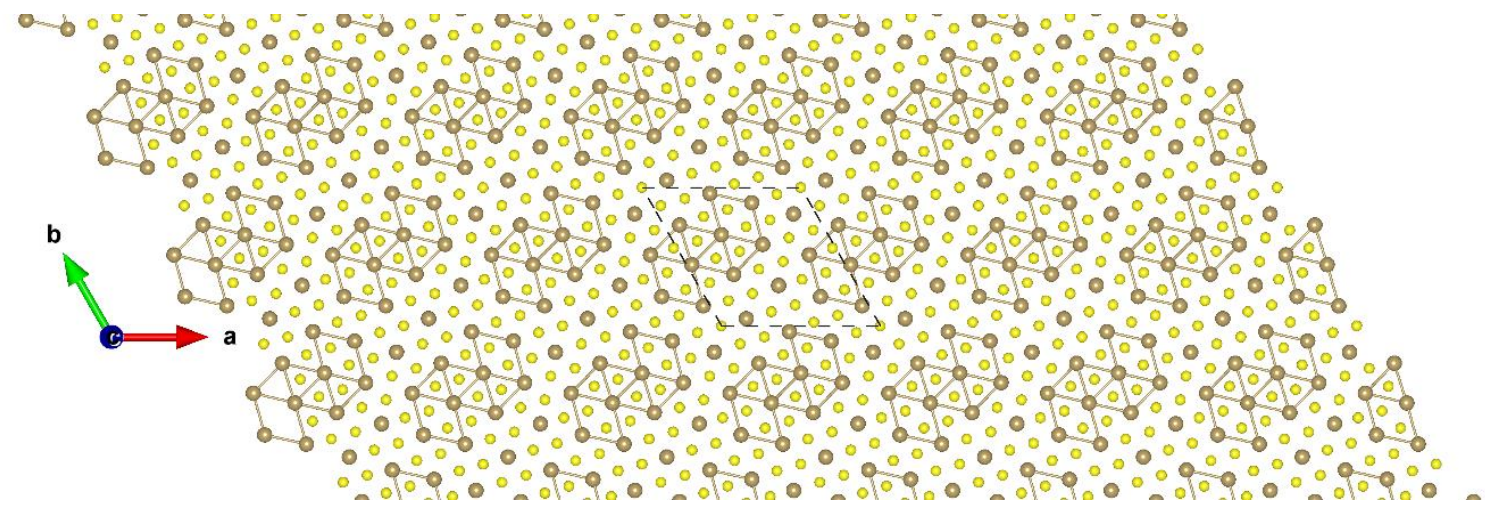

Figure S2: Top view of $\mathrm{TaS}_{2}-\mathrm{T} \sqrt{ } 13 \times \sqrt{ } 13 \phi-1$. 


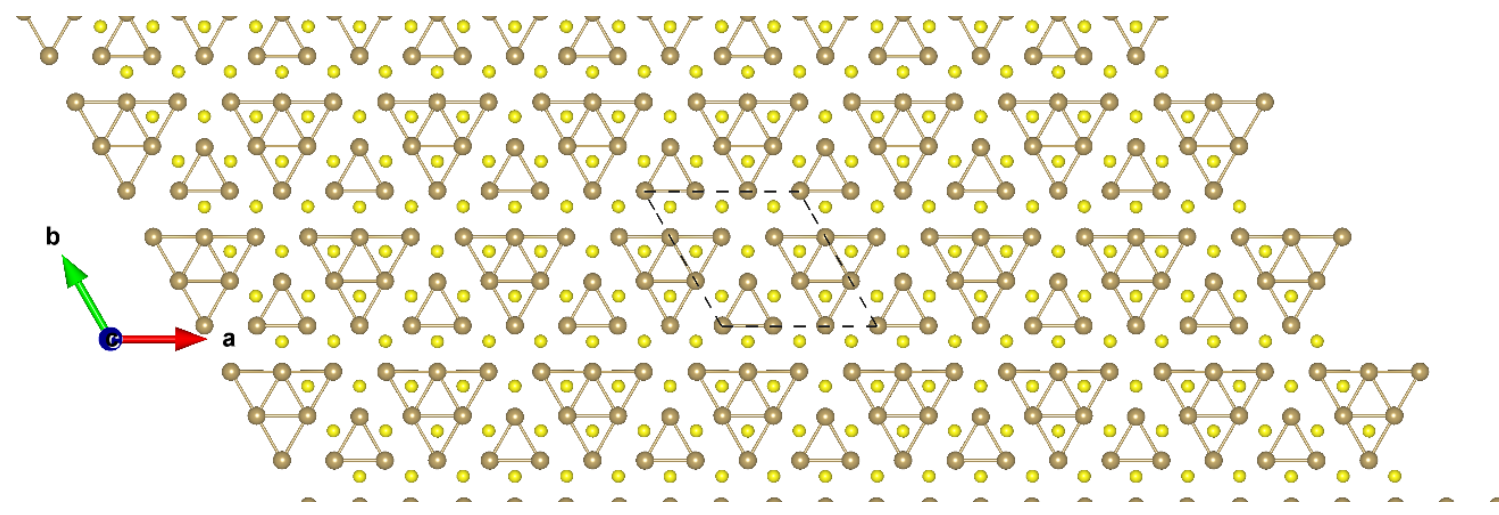

Figure S3: Top view of $\mathrm{TaS}_{2}-\mathrm{H} 3 \times 3 \phi_{-1}$.

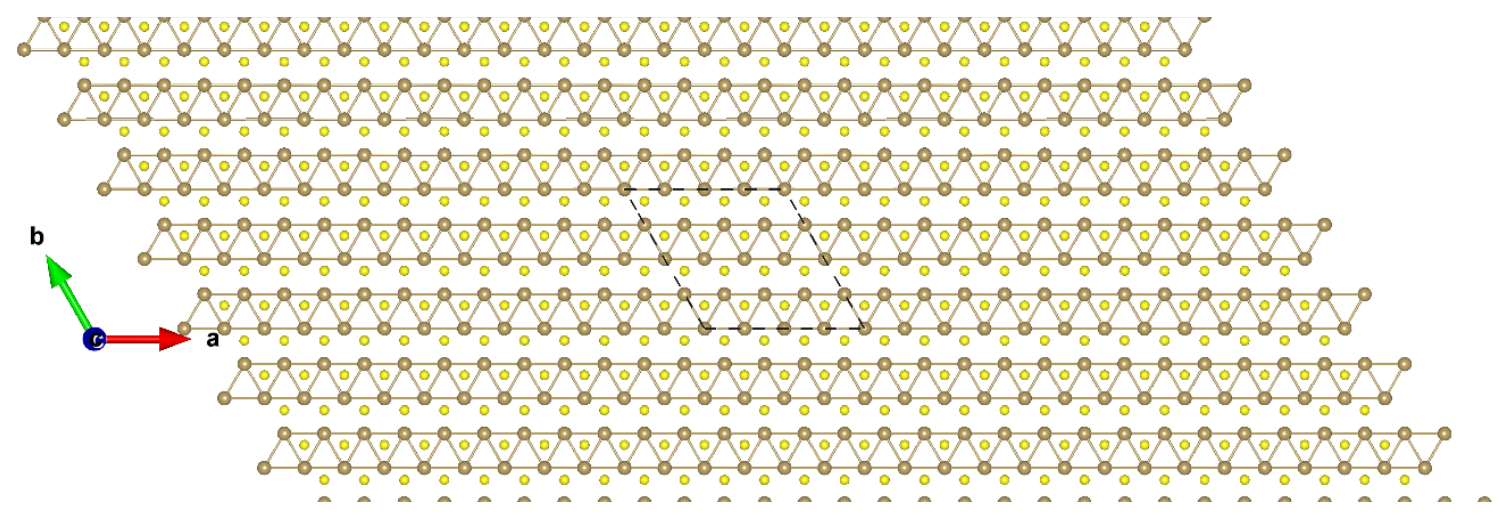

Figure S4: Top view of $\mathrm{TaS}_{2}-\mathrm{H} 4 \times 4 \phi-1$.

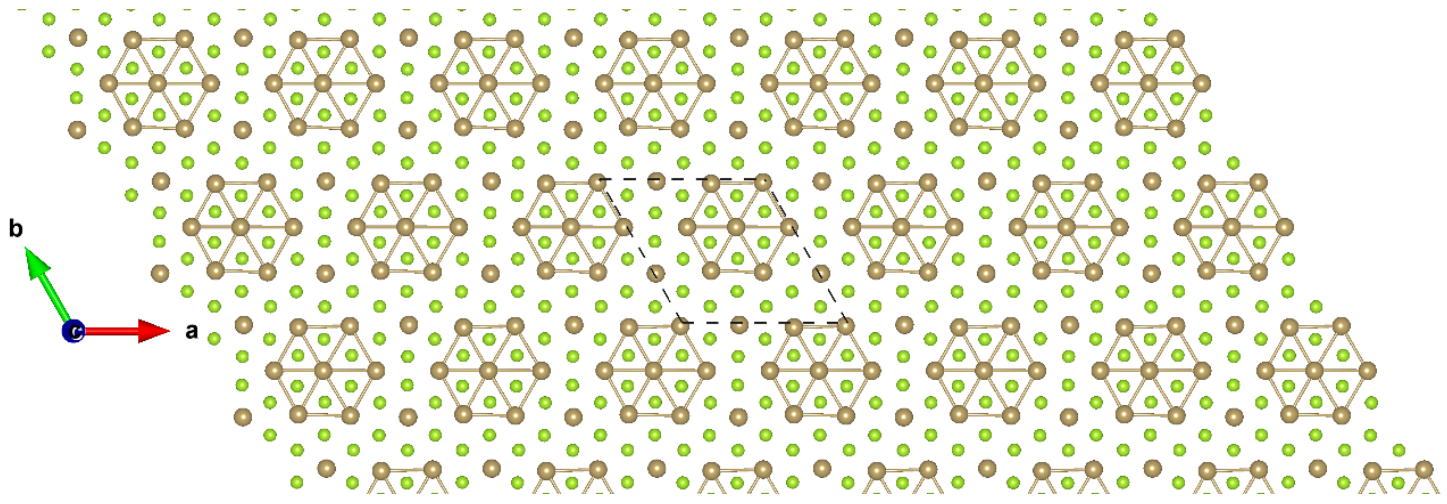

Figure S5: Top view of $\mathrm{TaSe}_{2}-\mathrm{T} 3 \times 3 \phi_{-1}$. The brown and green balls represent the $\mathrm{Ta}$ and $\mathrm{Se}$ atoms, respectively. 


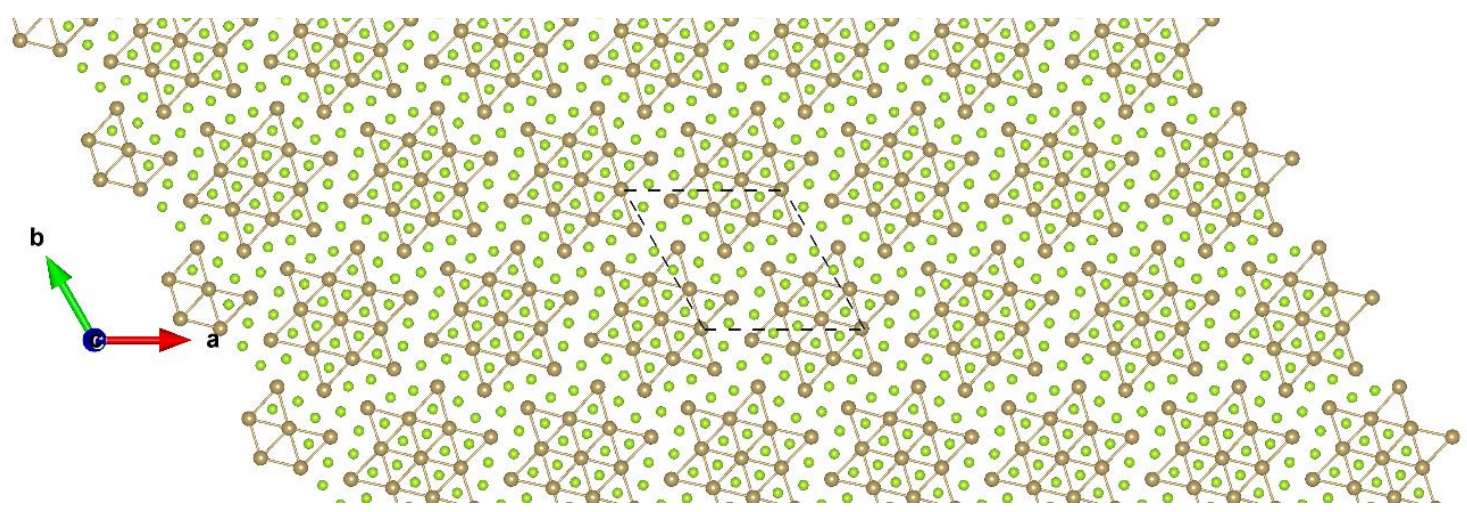

Figure S6: Top view of $\mathrm{TaSe}_{2}-\mathrm{T} \sqrt{ } 13 \times \sqrt{13} \phi_{-1}$.

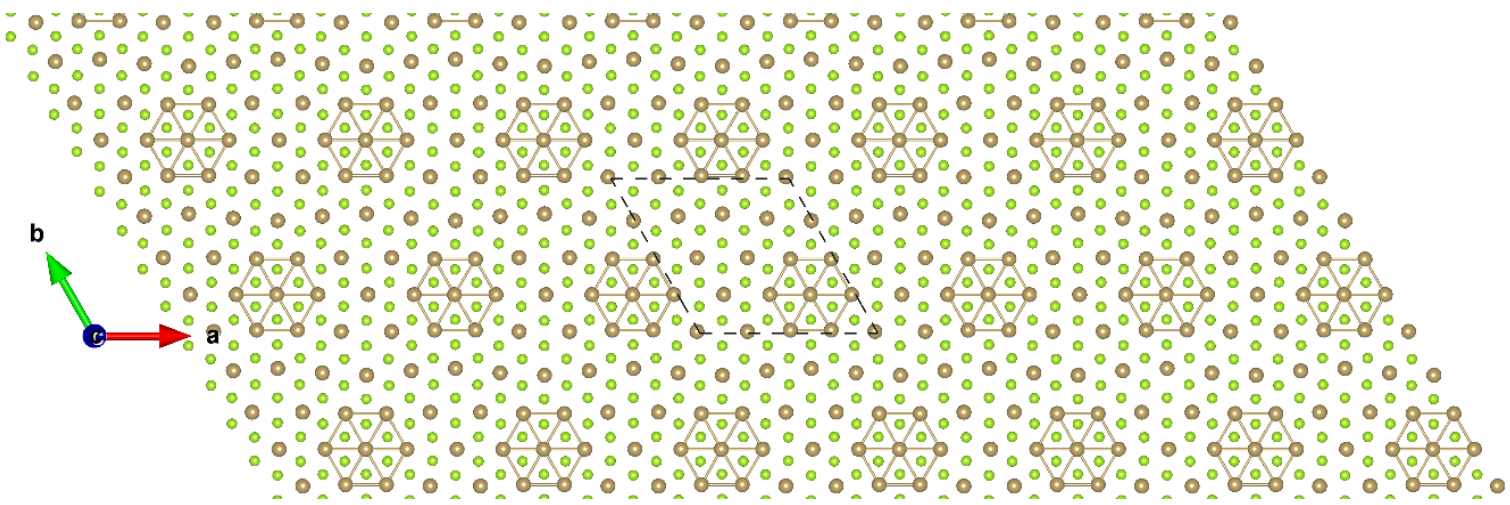

Figure S7: Top view of $\mathrm{TaSe}_{2}-\mathrm{T} 4 \times 4 \phi-1$.

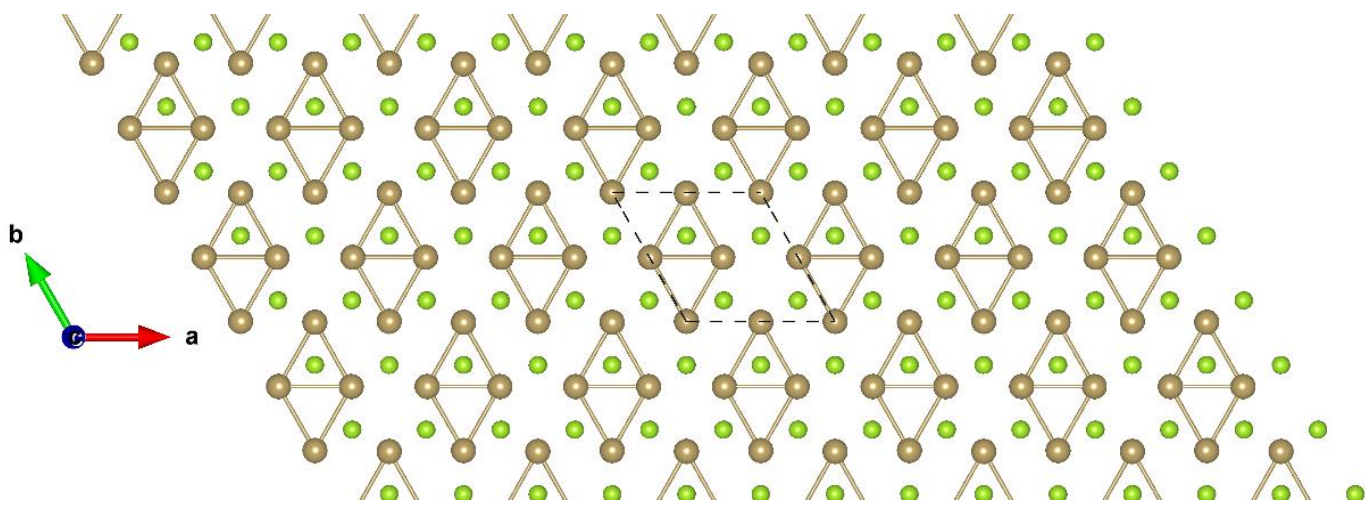

Figure S8: Top view of $\mathrm{TaSe}_{2}-\mathrm{H} 2 \times 2 \phi-1$. 


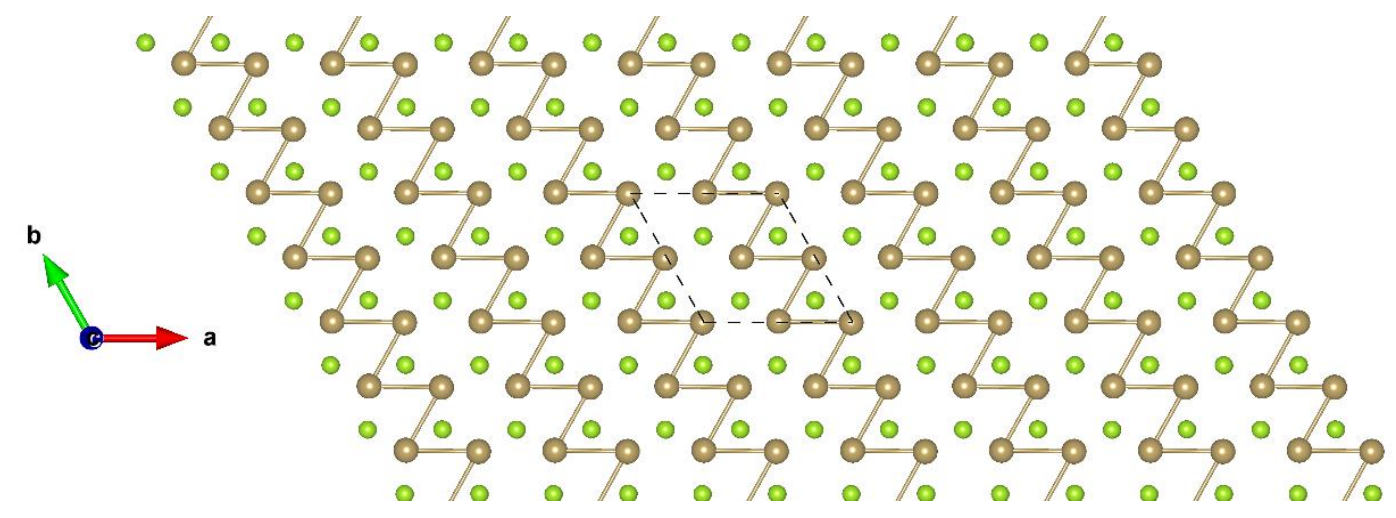

Figure S9: Top view of $\mathrm{TaSe}_{2}-\mathrm{H} 2 \times 2 \phi_{-2}$.

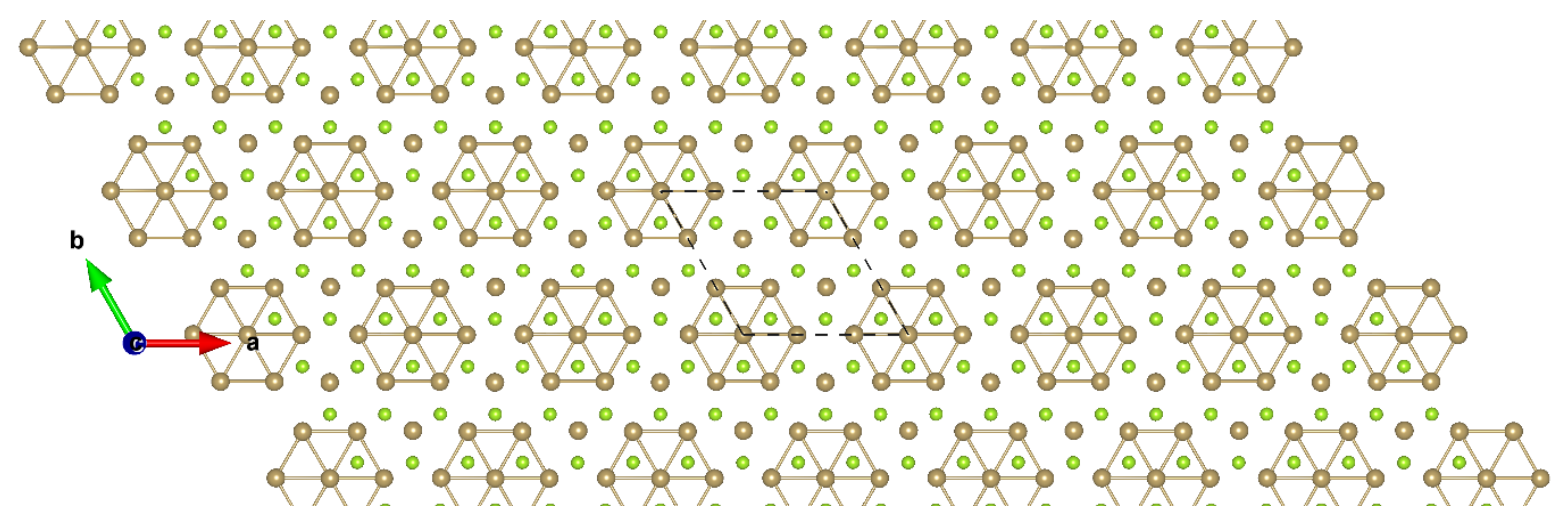

Figure S10: Top view of $\mathrm{TaSe}_{2}-\mathrm{H} 3 \times 3 \phi_{-1}$.

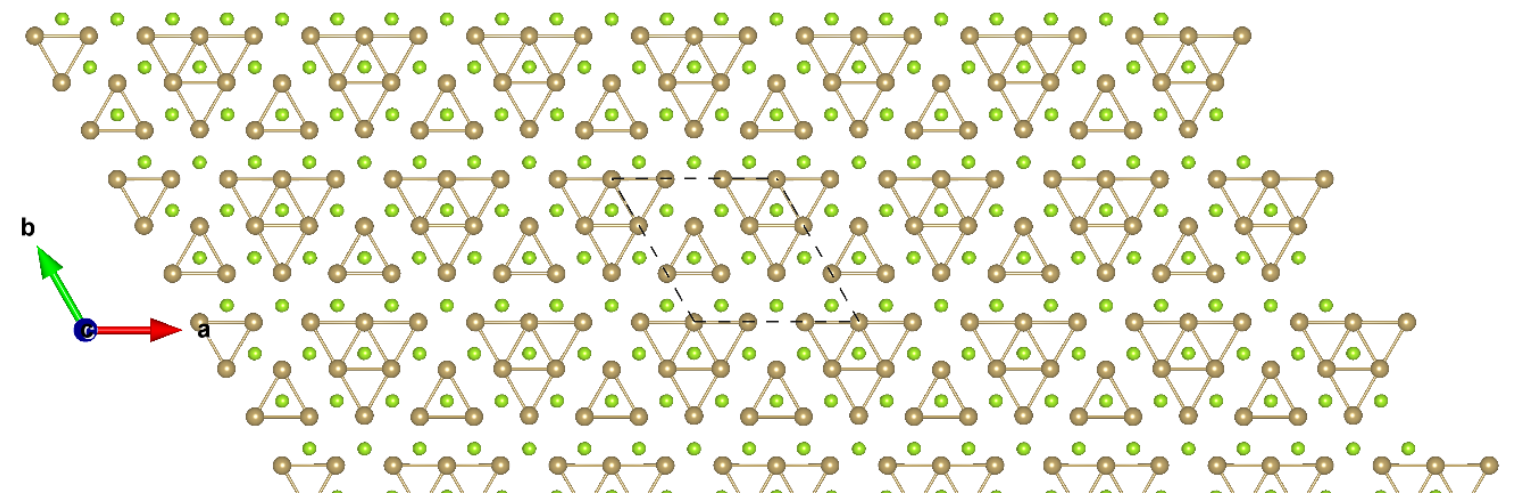

Figure S11: Top view of $\mathrm{TaSe}_{2}-\mathrm{H} 3 \times 3 \phi_{-2}$. 


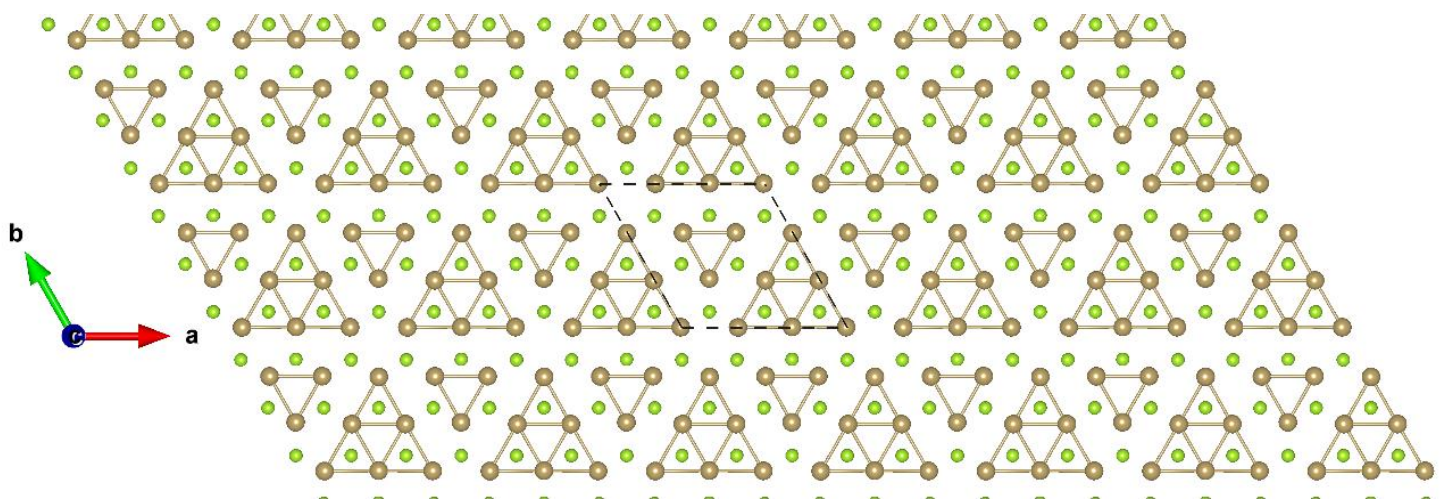

Figure S12: Top view of $\mathrm{TaSe}_{2}-\mathrm{H} 3 \times 3 \phi-3$.

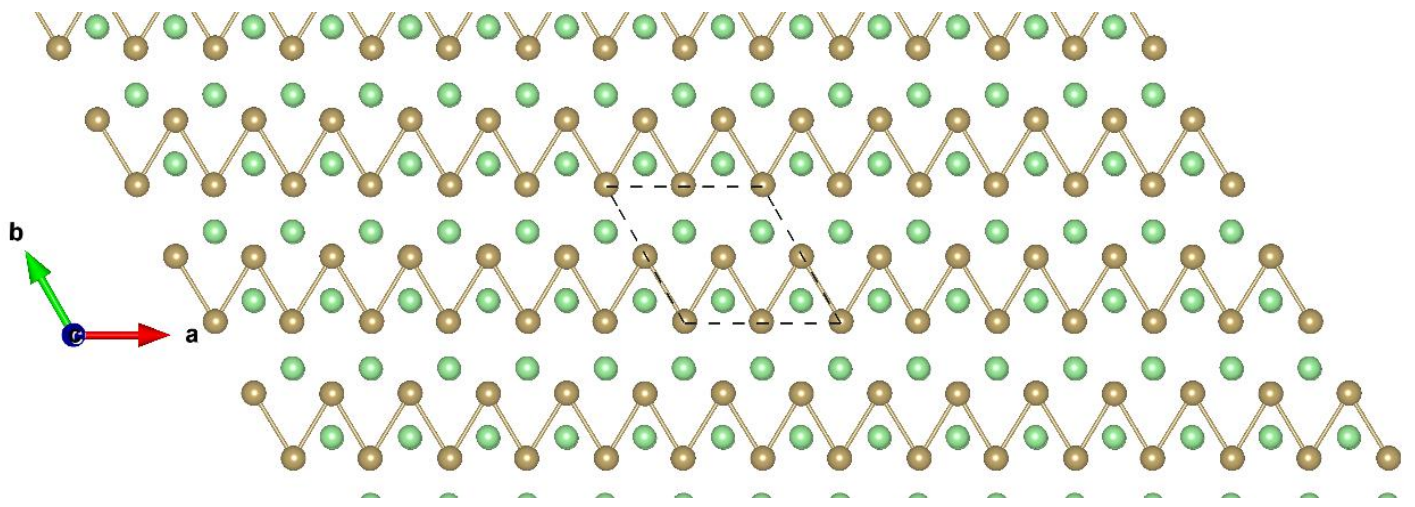

Figure S13: Top view of $\mathrm{TaTe}_{2}-\mathrm{H} 2 \times 2 \phi_{-1}$. The brown and dark green balls represent the Ta and Te atoms, respectively.

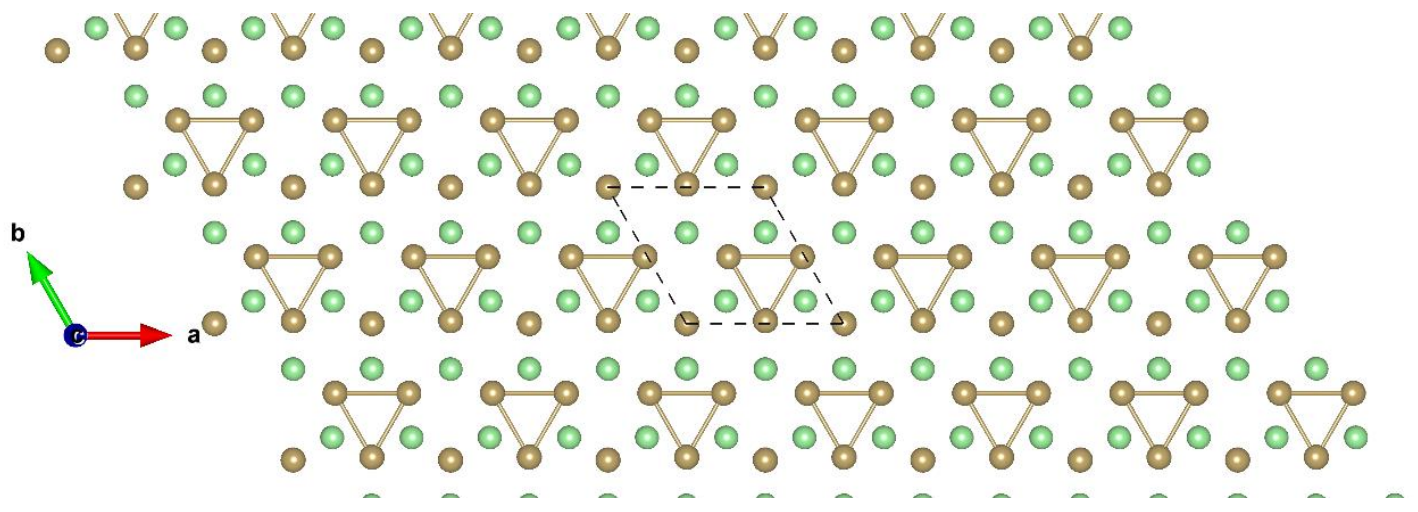

Figure S14: Top view of $\mathrm{TaTe}_{2}-\mathrm{H} 2 \times 2 \phi_{-2}$. 


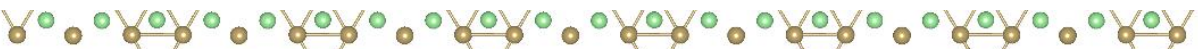

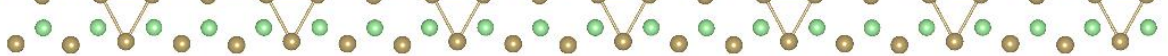

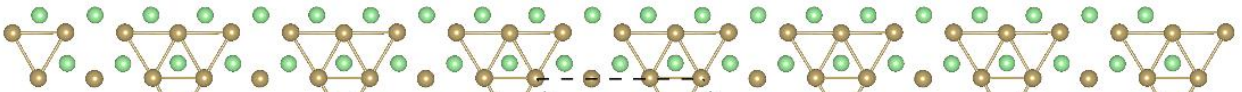

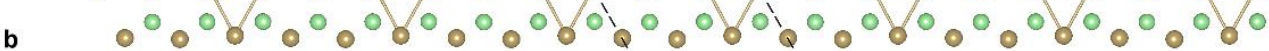

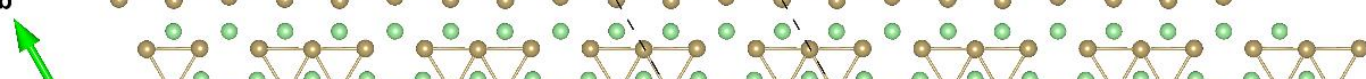

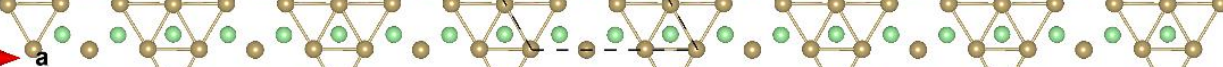

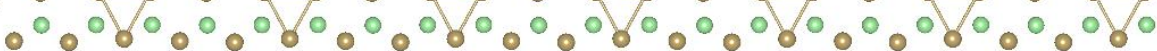

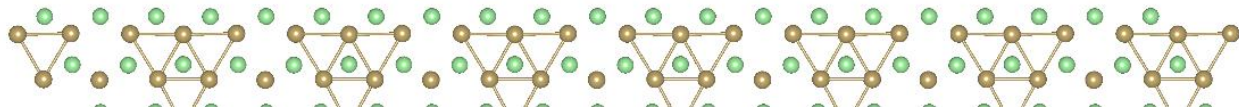

Figure S15: Top view of $\mathrm{TaTe}_{2}-\mathrm{H} 3 \times 3 \phi_{-1}$.

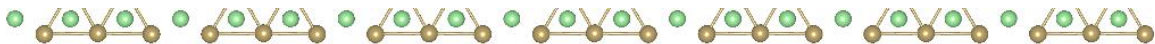

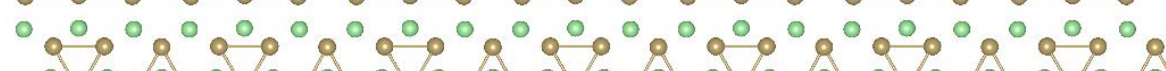

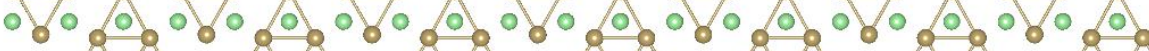

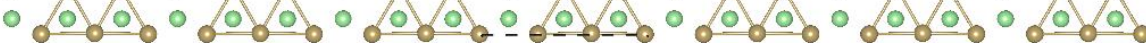

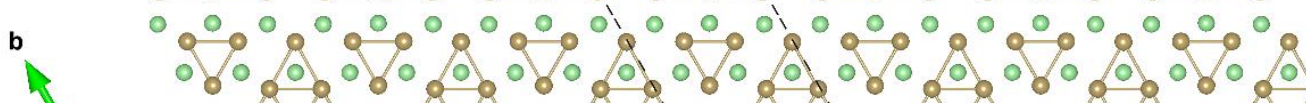

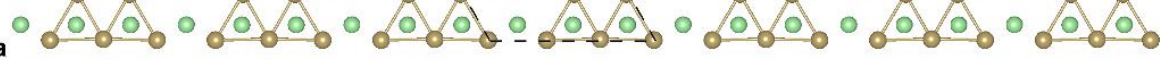

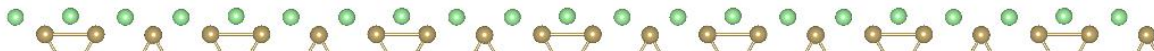

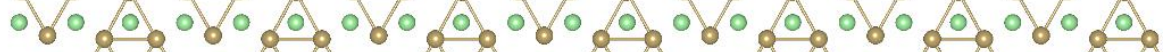

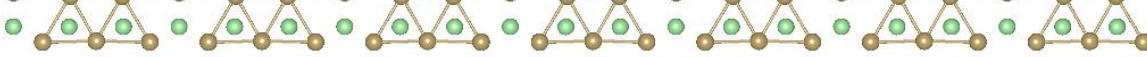

Figure S16: Top view of $\mathrm{TaTe}_{2}-\mathrm{H} 3 \times 3 \phi-2$.

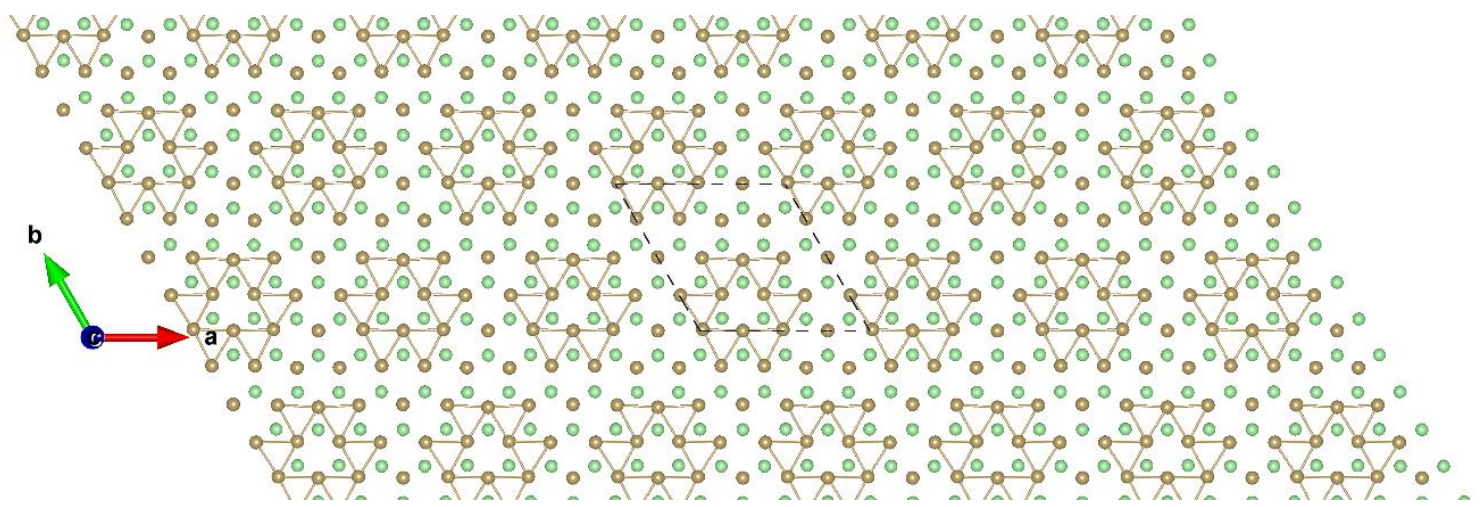

Figure S17: Top view of $\mathrm{TaTe}_{2}-\mathrm{H} 4 \times 4 \phi_{-1}$. 


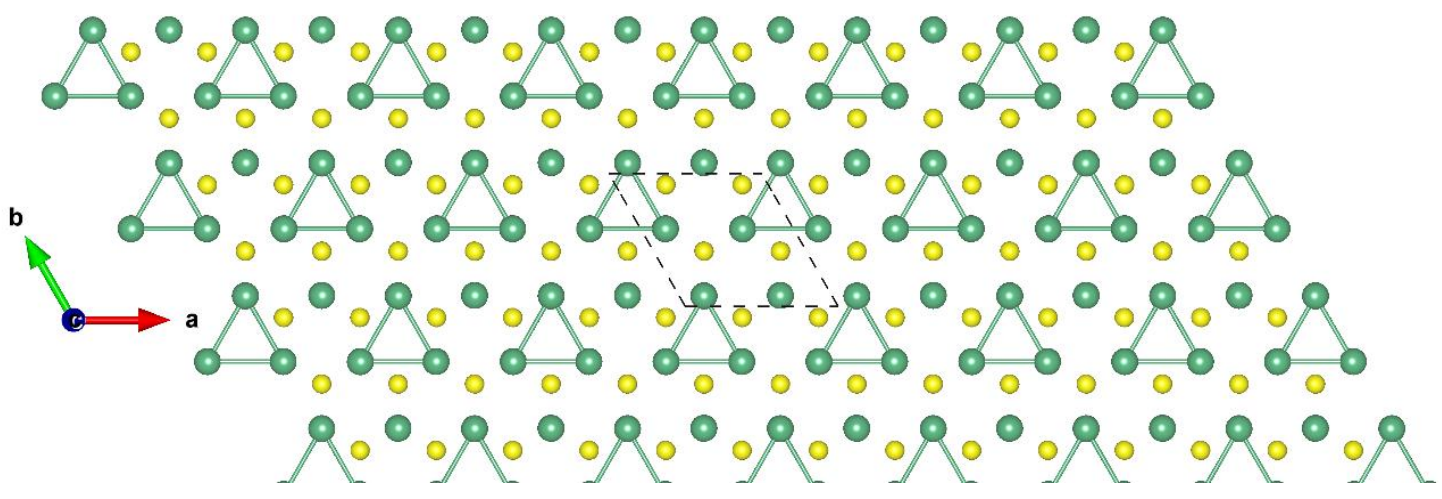

Figure S18: Top view of $\mathrm{NbS}_{2}-\mathrm{H} 2 \times 2 \phi-1$. The dark green and yellow balls represent the $\mathrm{Nb}$ and $\mathrm{S}$ atoms, respectively.

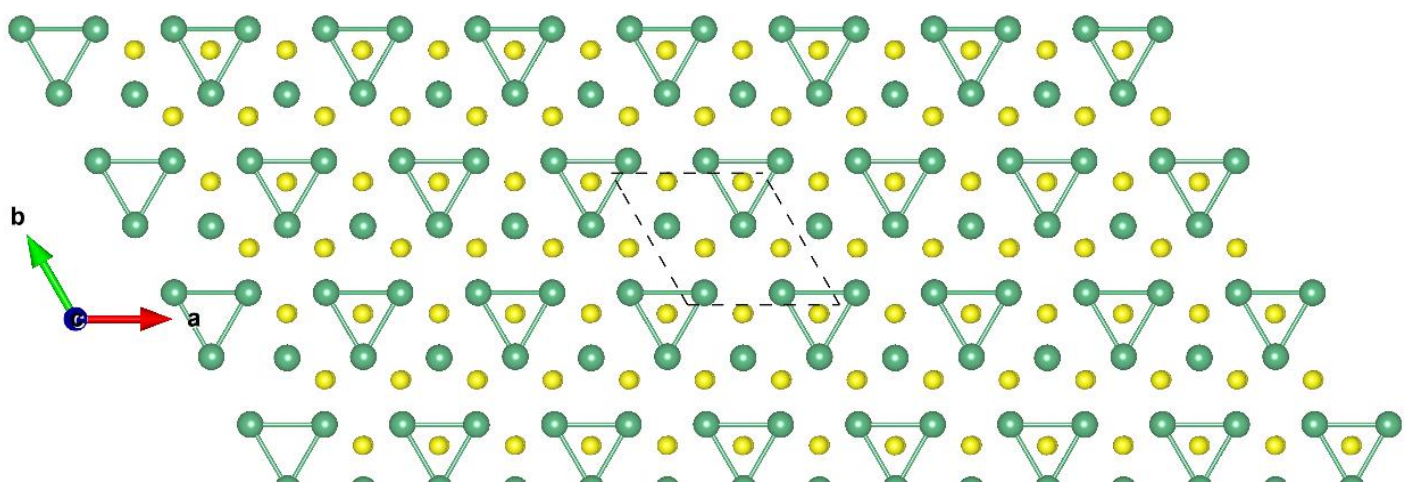

Figure S19: Top view of $\mathrm{NbS}_{2}-\mathrm{H} 2 \times 2 \phi-2$.

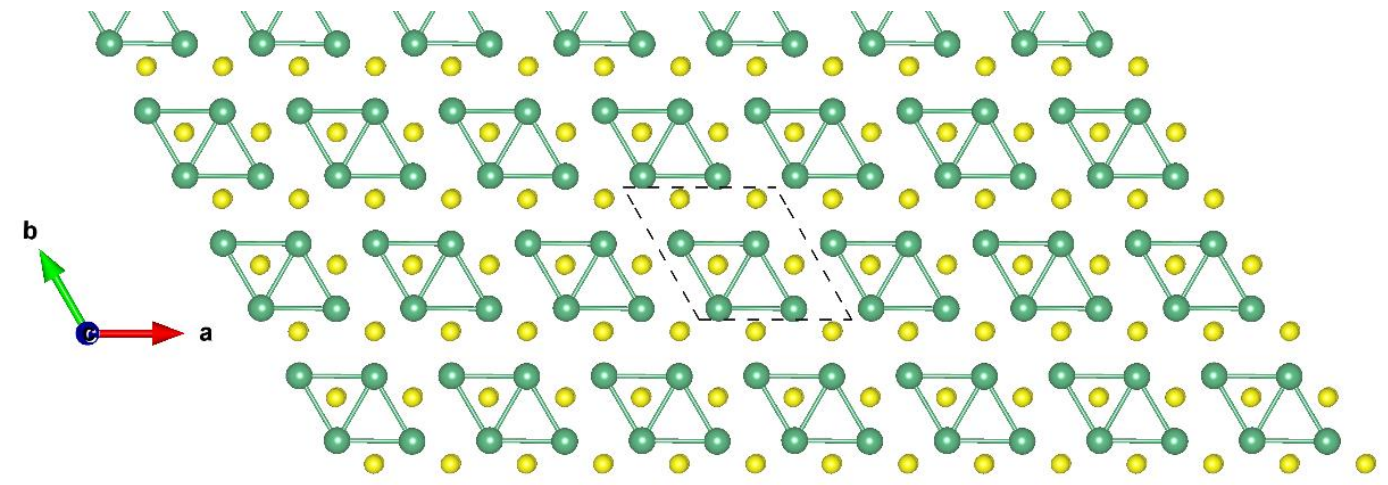

Figure S20: Top view of $\mathrm{NbS}_{2}-\mathrm{H} 2 \times 2 \phi_{-3}$. 


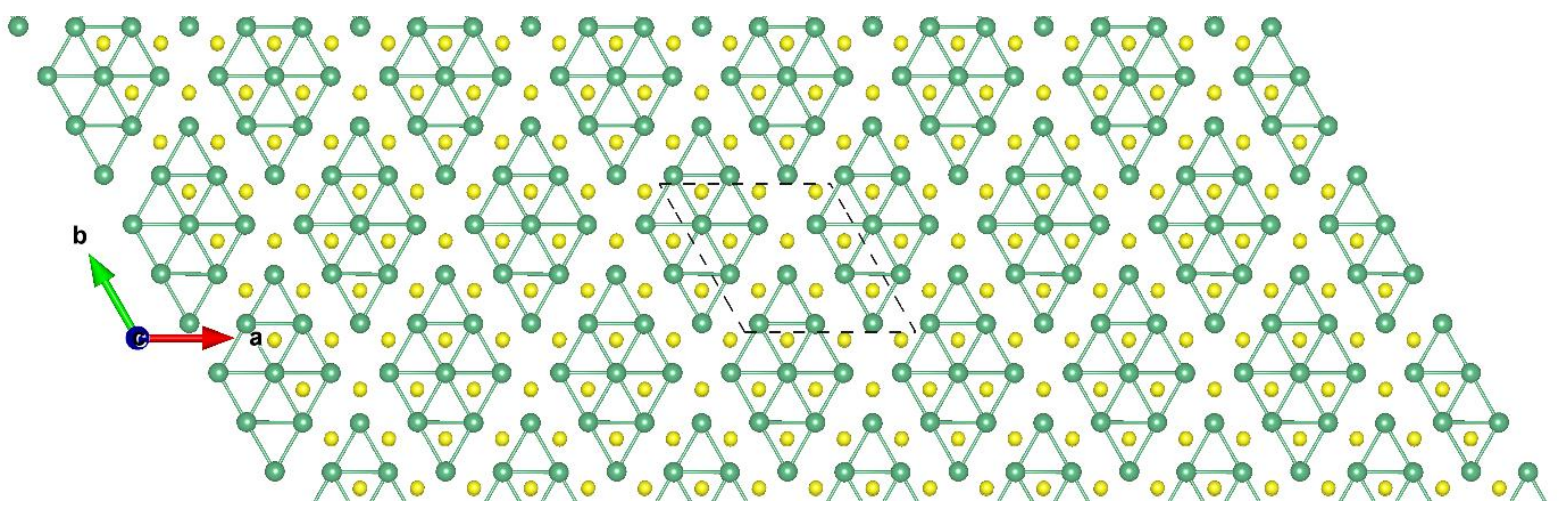

Figure S21: Top view of $\mathrm{NbS}_{2}-\mathrm{H} 3 \times 3 \phi-1$.

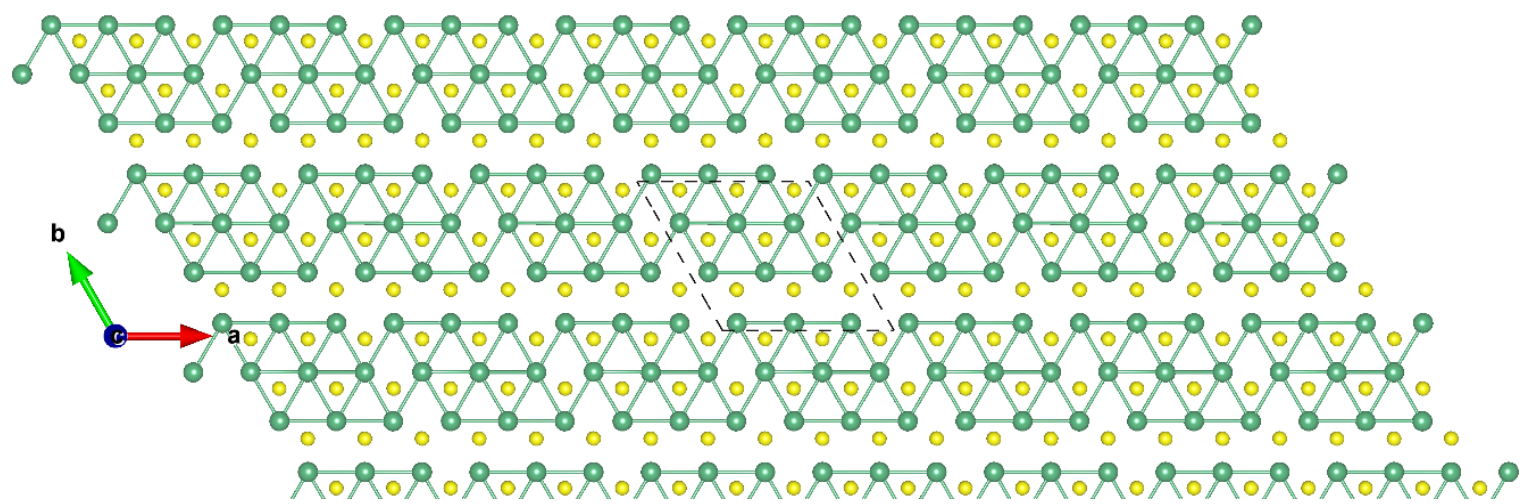

Figure S22: Top view of $\mathrm{NbS}_{2}-\mathrm{H} 3 \times 3 \phi$-2.

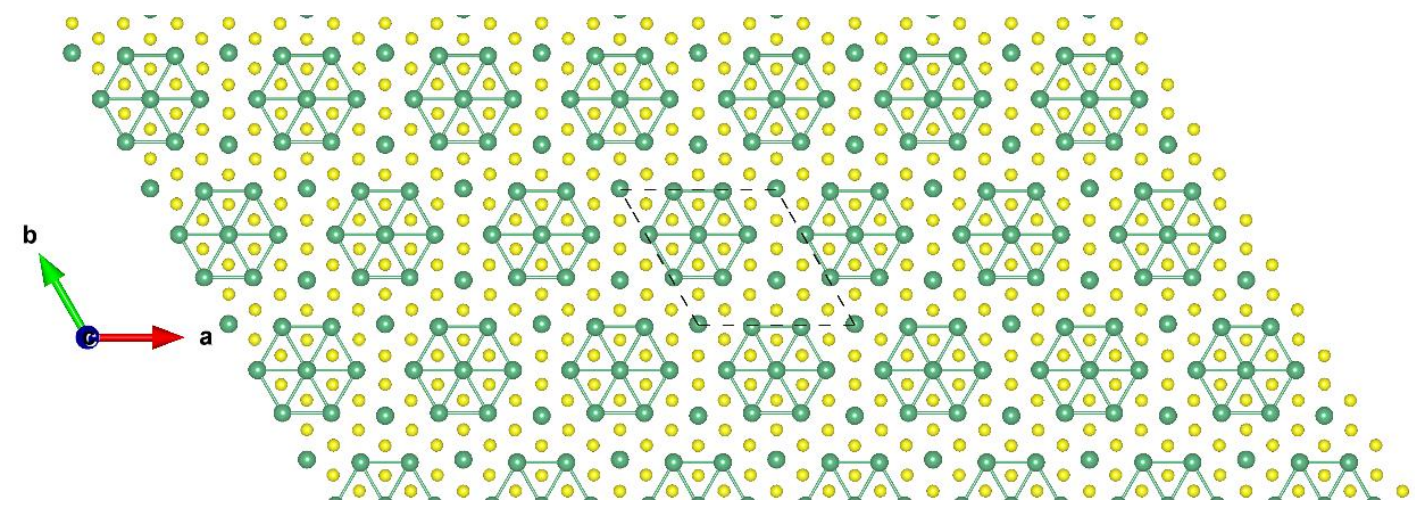

Figure S23: Top view of $\mathrm{NbS}_{2}-\mathrm{T} 3 \times 3 \phi_{-1}$. 


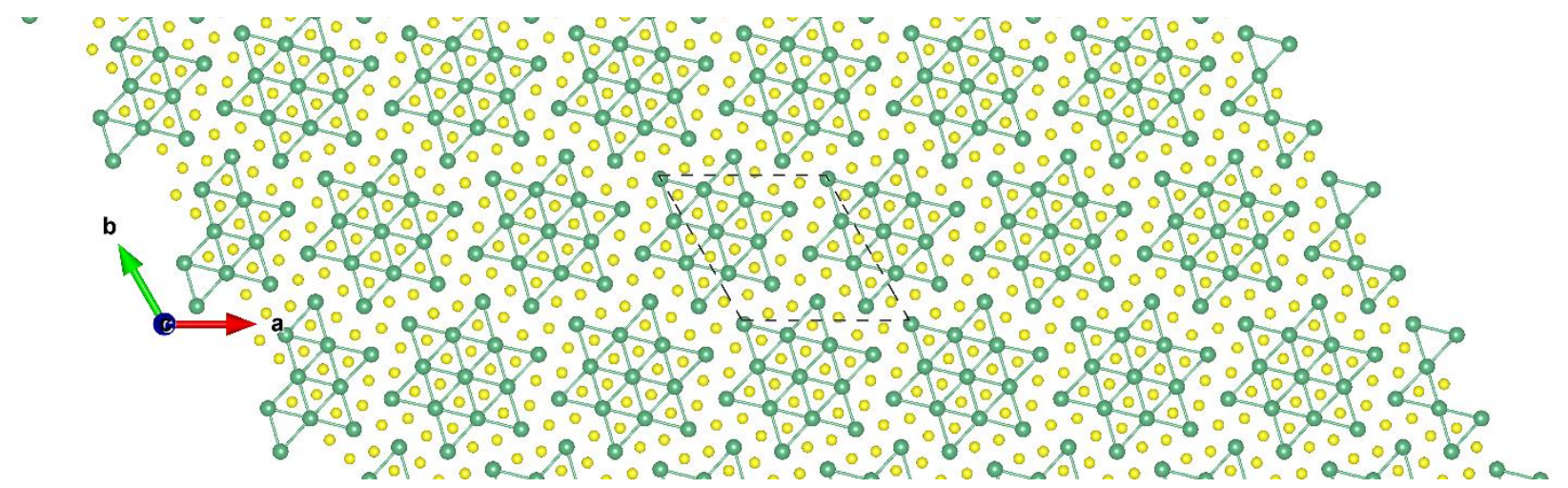

Figure S24: Top view of $\mathrm{NbS}_{2}-\mathrm{T} \sqrt{ } 13 \times \sqrt{ } 13 \phi_{-1}$.

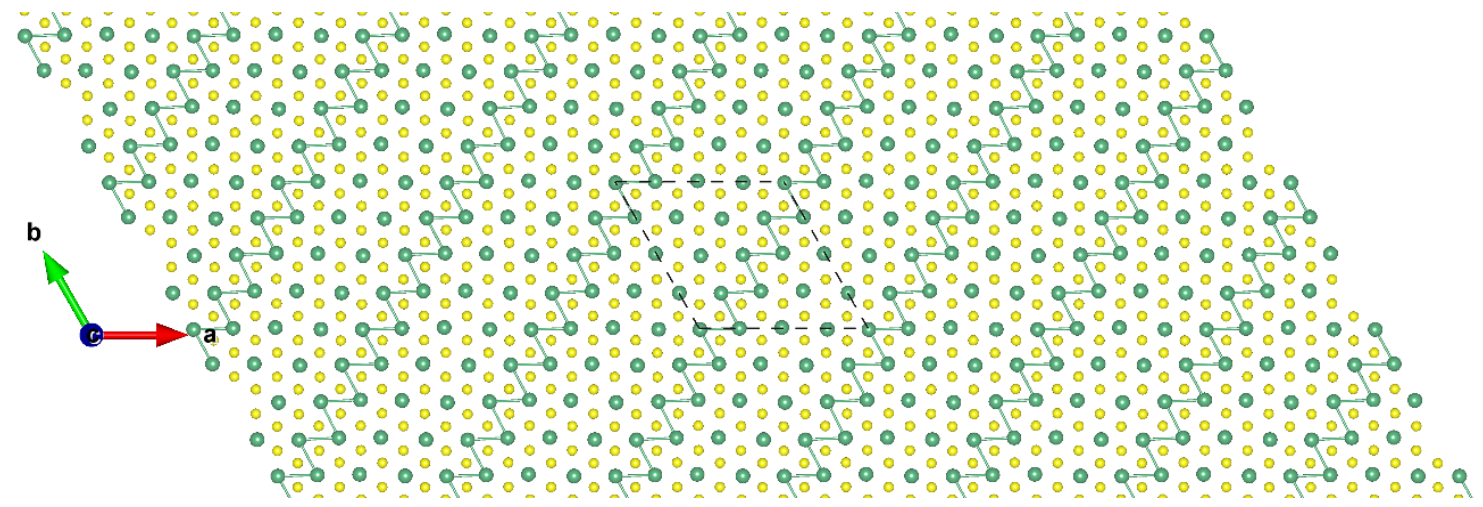

Figure S25: Top view of $\mathrm{NbS}_{2}-\mathrm{T} 4 \times 4 \phi_{-1}$.

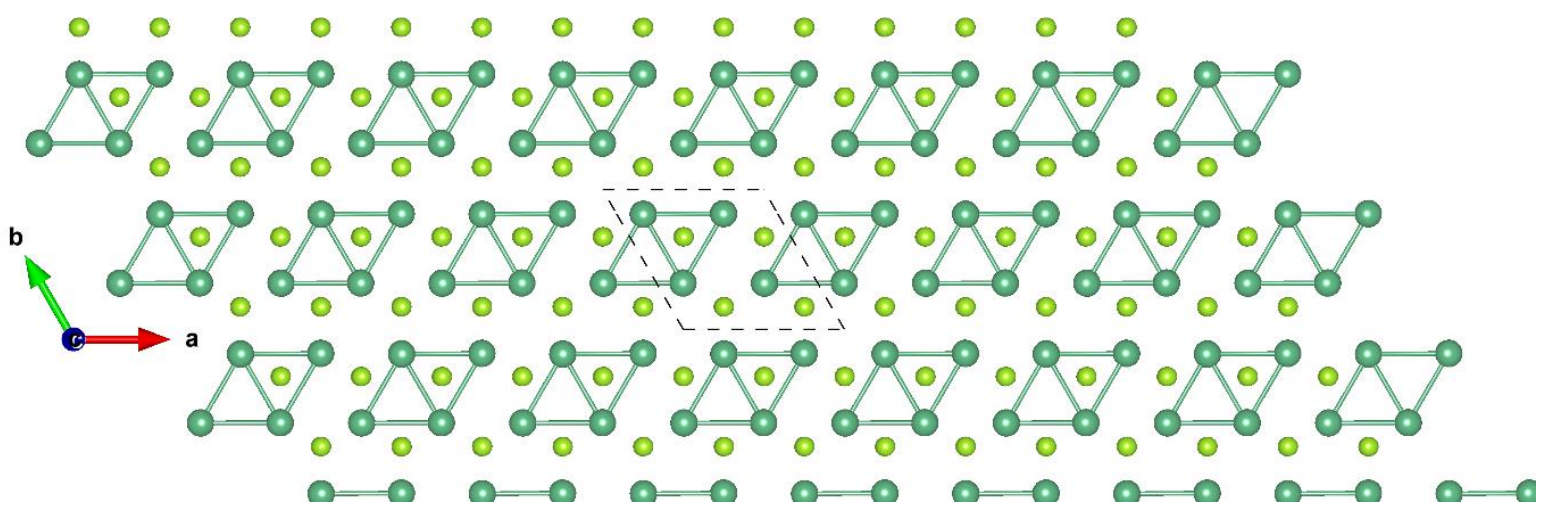

Figure S26: Top view of $\mathrm{NbSe}_{2}-\mathrm{H} 2 \times 2 \phi_{-1}$. The dark and light green balls represent the $\mathrm{Nb}$ and $\mathrm{Se}$ atoms, respectively. 


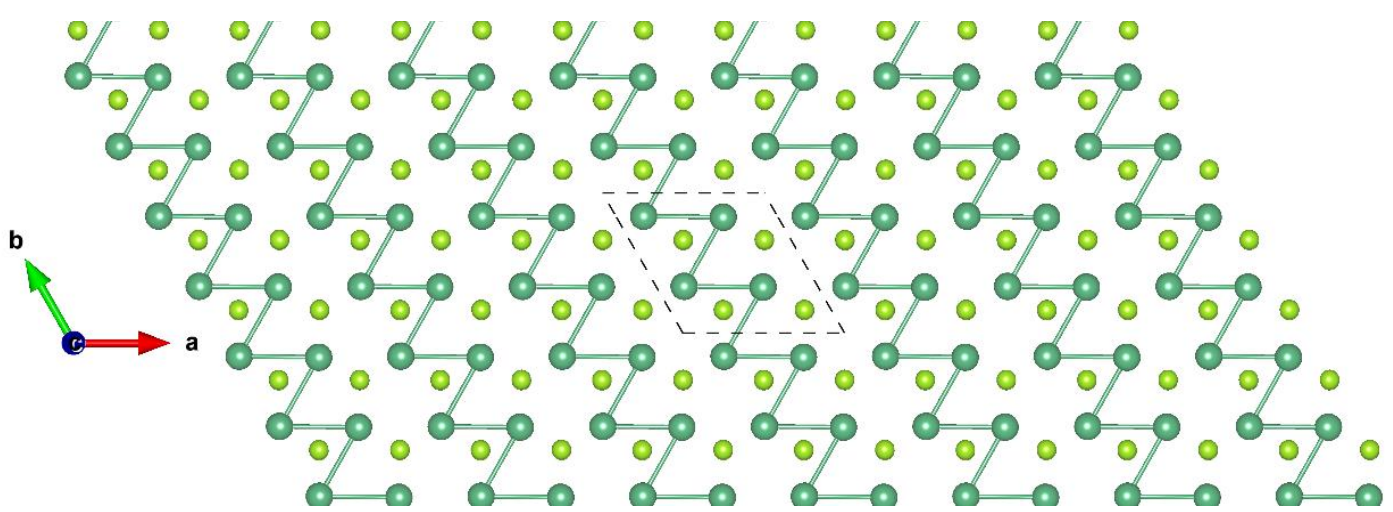

Figure S27: Top view of $\mathrm{NbSe}_{2}-\mathrm{H} 2 \times 2 \phi-2$.

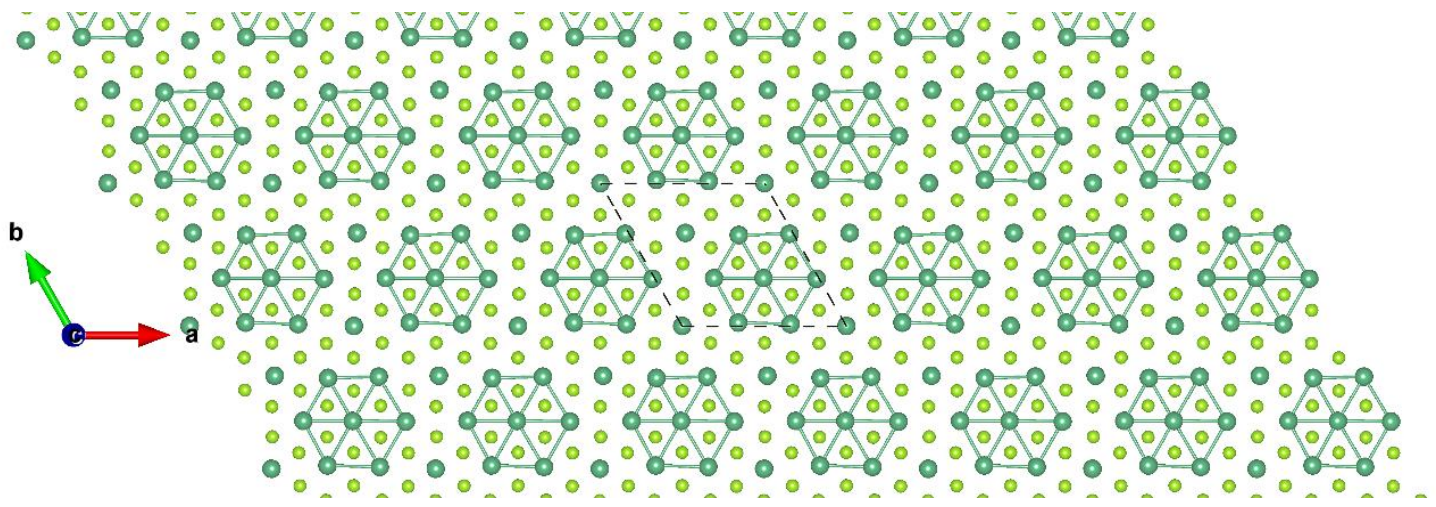

Figure S28: Top view of $\mathrm{NbSe}_{2}-\mathrm{T} 3 \times 3 \phi_{-1}$.

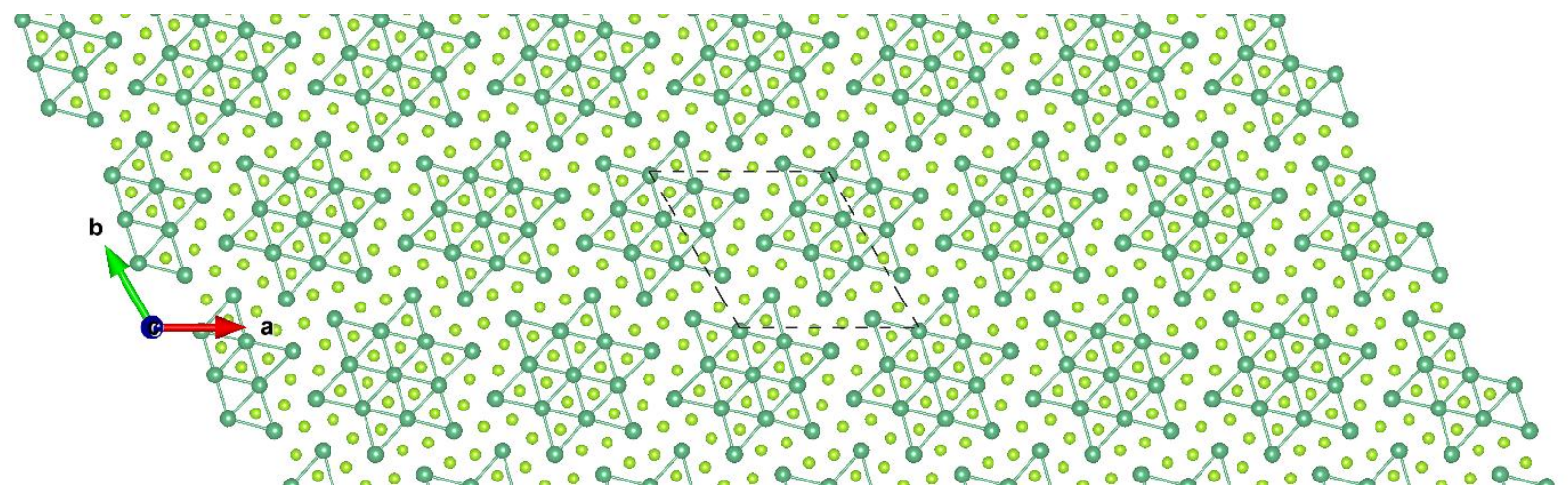

Figure S29: Top view of $\mathrm{NbSe}_{2}-\mathrm{T} \sqrt{13} \times \sqrt{13} \phi-1$. 


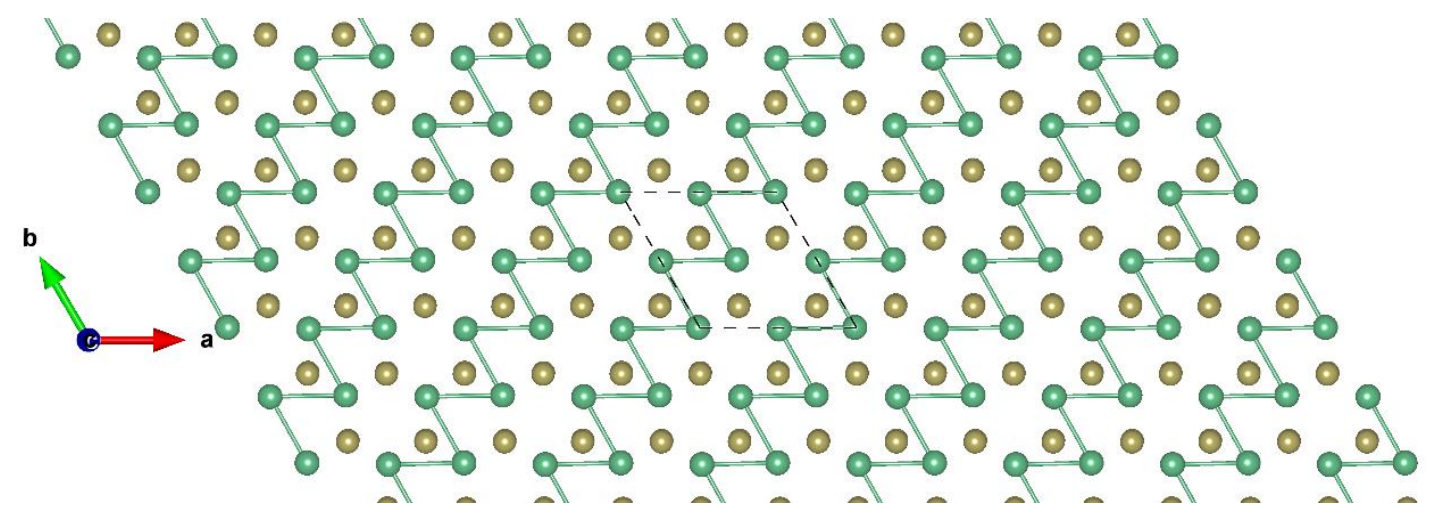

Figure S30: Top view of $\mathrm{NbTe}_{2}-\mathrm{H} 2 \times 2 \phi_{-1}$. The dark green and brown balls represent the $\mathrm{Nb}$ and Se atoms, respectively.

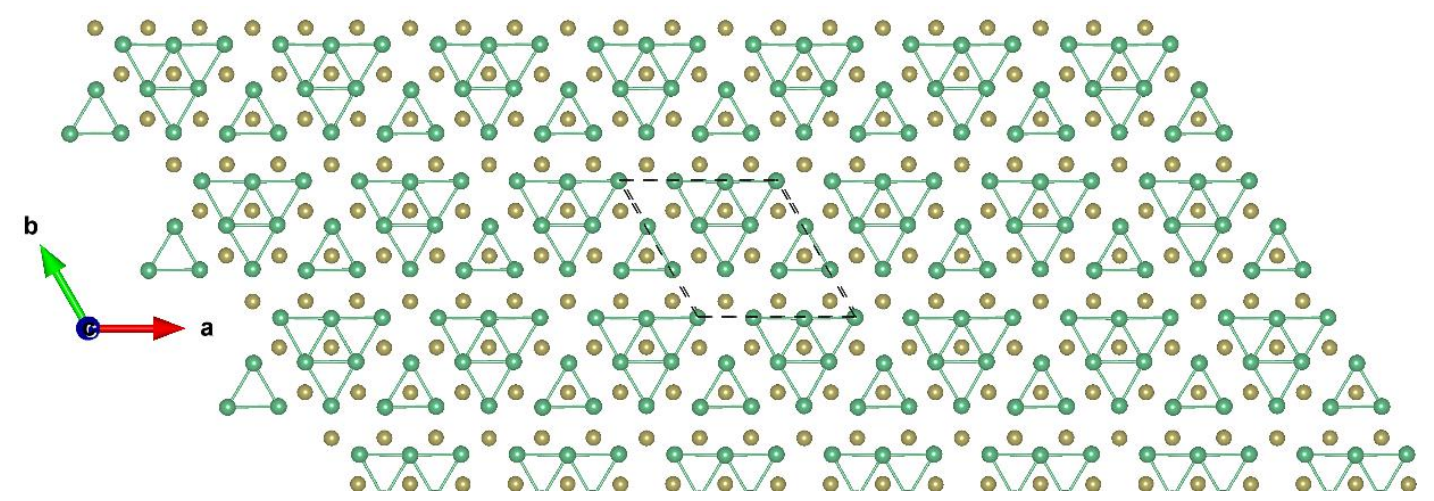

Figure S31: Top view of $\mathrm{NbTe}_{2}-\mathrm{H} 3 \times 3 \phi_{-1}$.

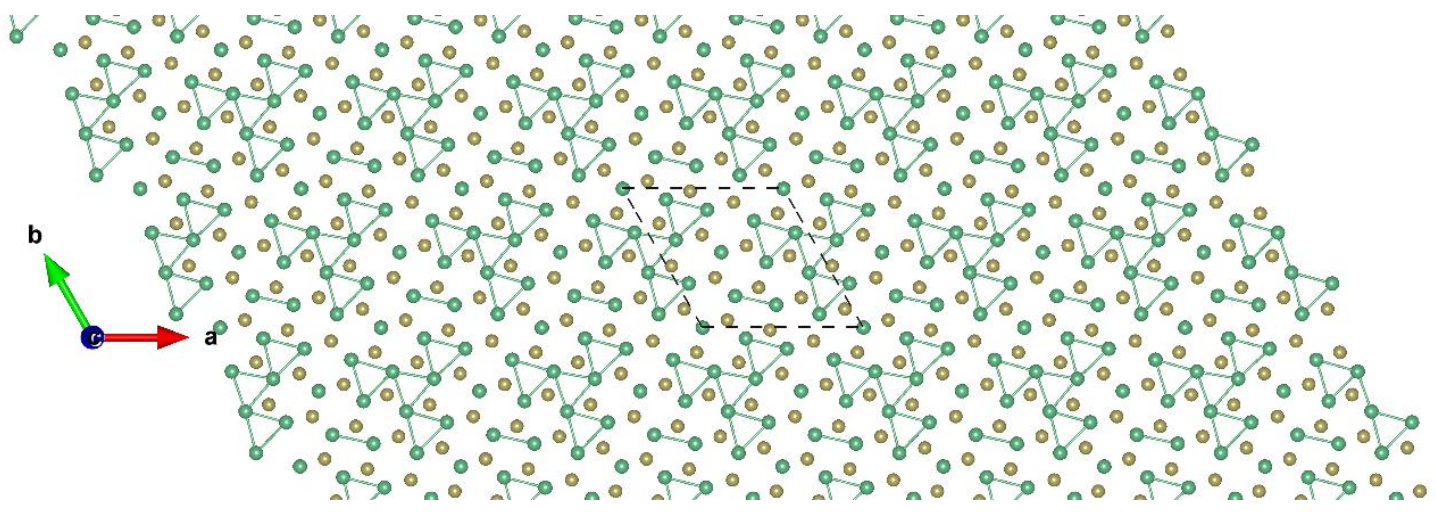

Figure S32: Top view of $\mathrm{NbTe}_{2}-\mathrm{H} \sqrt{ } 13 \times \sqrt{ } 13 \phi-1$. 


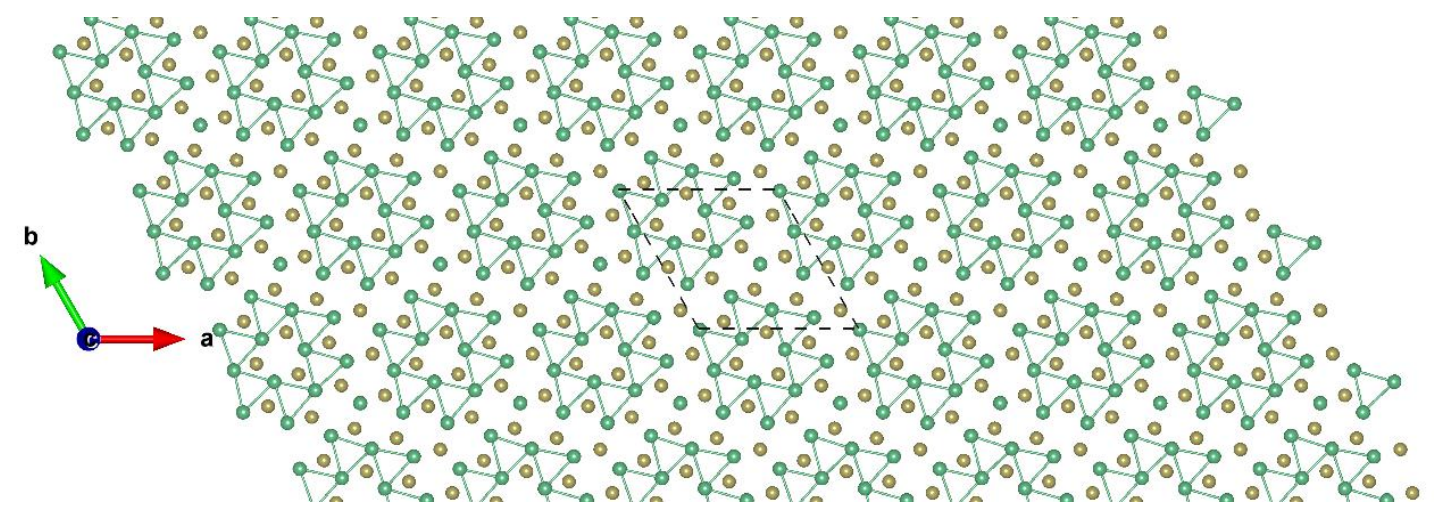

Figure S33: Top view of $\mathrm{NbTe}_{2}-\mathrm{H} \sqrt{ } 13 \times \sqrt{ } 13 \phi-2$.

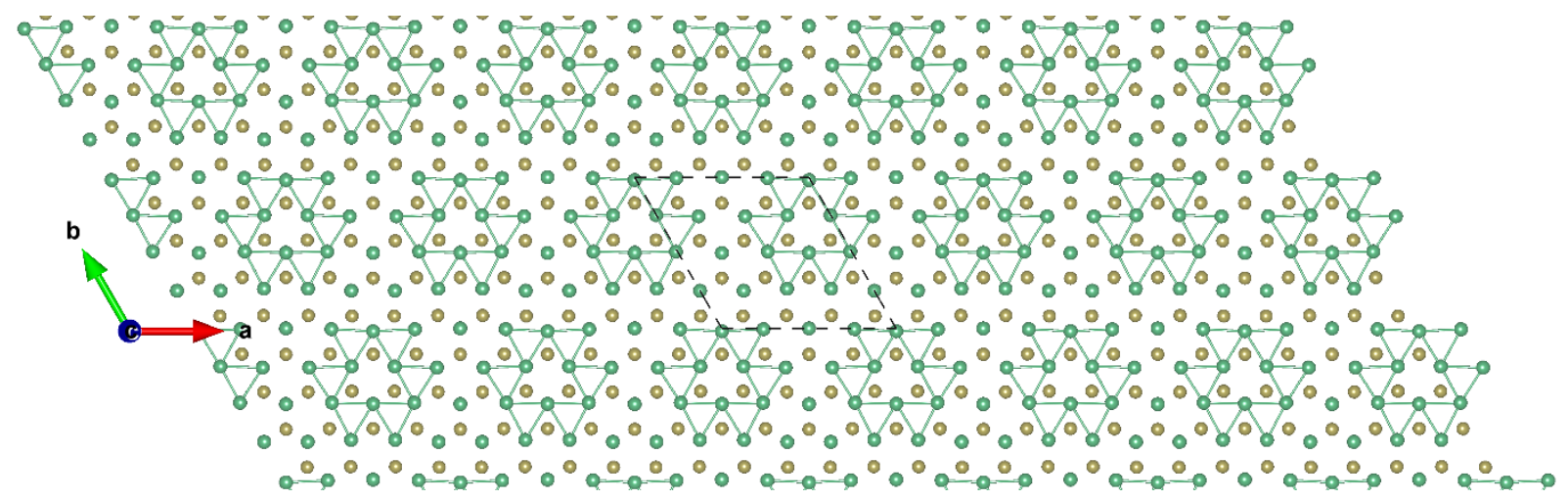

Figure S34: Top view of $\mathrm{NbTe}_{2}-\mathrm{H} 4 \times 4 \phi-1$.

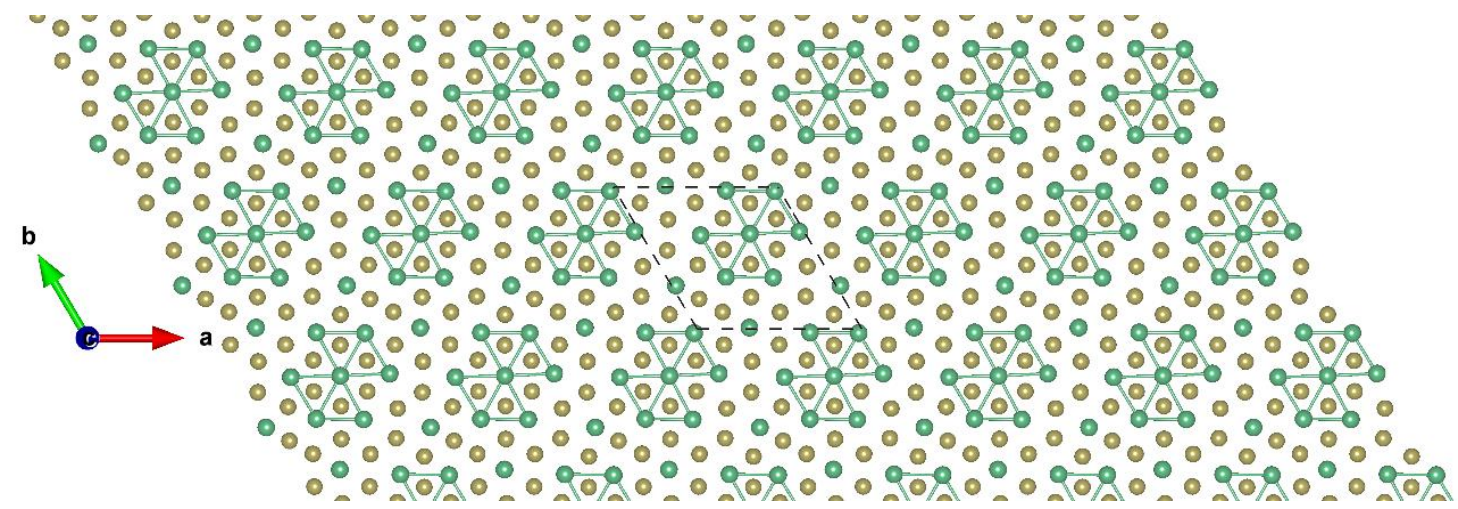

Figure S35: Top view of $\mathrm{NbTe}_{2}-\mathrm{T} 3 \times 3 \phi-1$. 


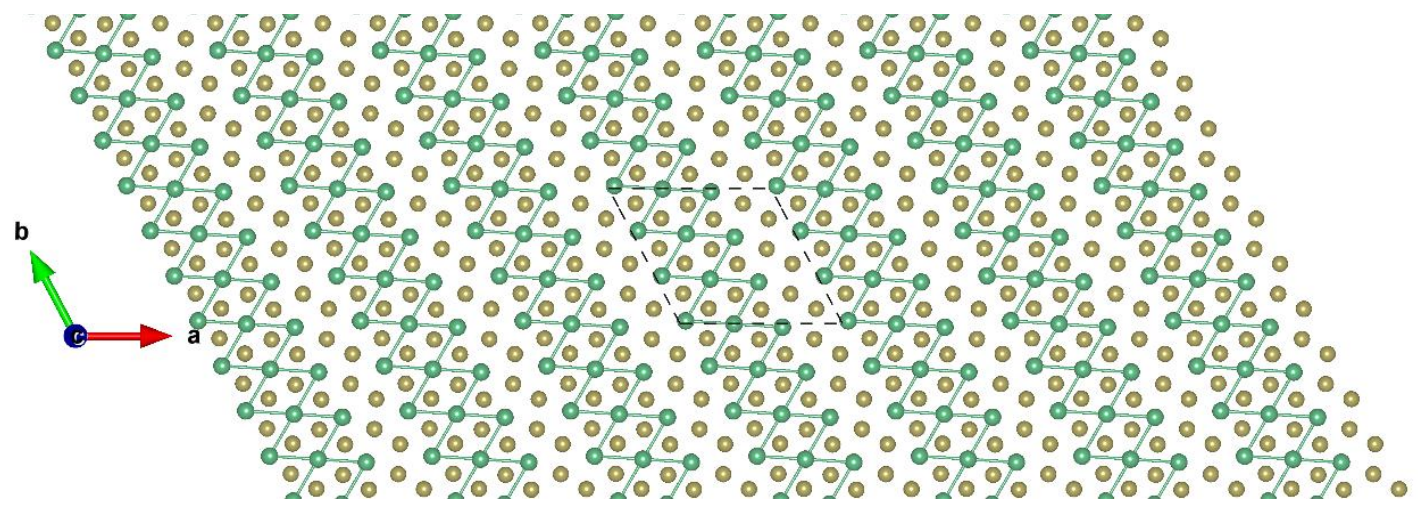

Figure S36: Top view of $\mathrm{NbTe}_{2}-\mathrm{T} 3 \times 3 \phi-2$.

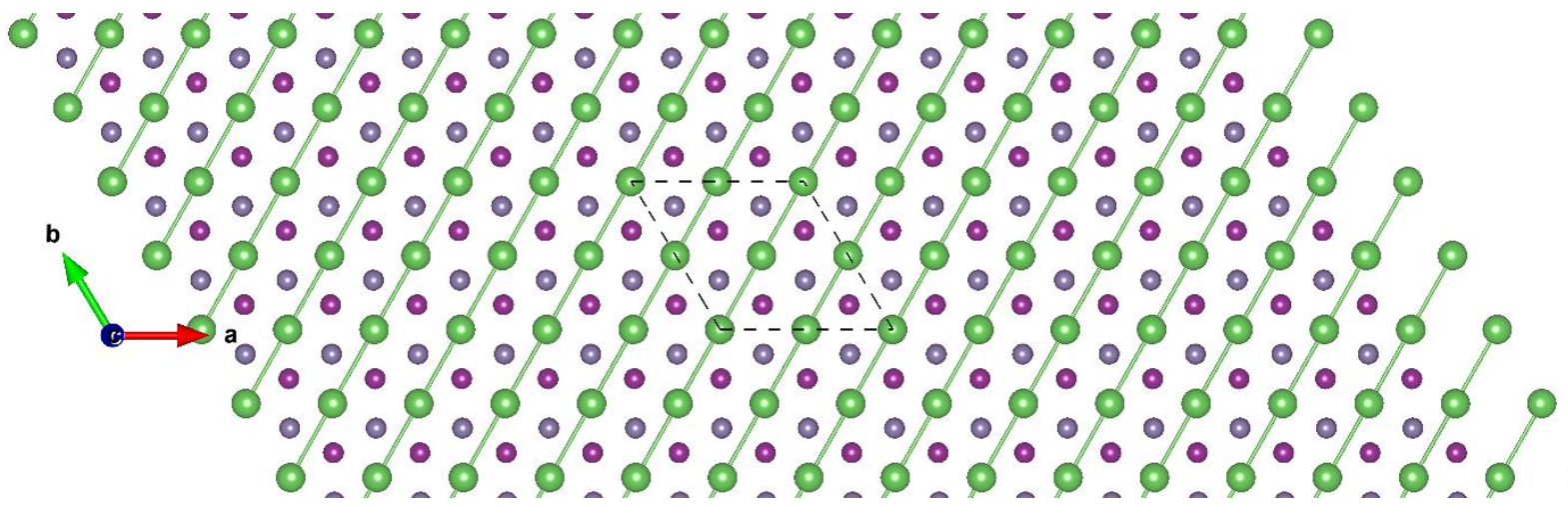

Figure S37: Top view of LaGeI $2 \times 2 \phi_{-1}$. The dark green, blue and purple balls represent the La, Ge, and I atoms, respectively.

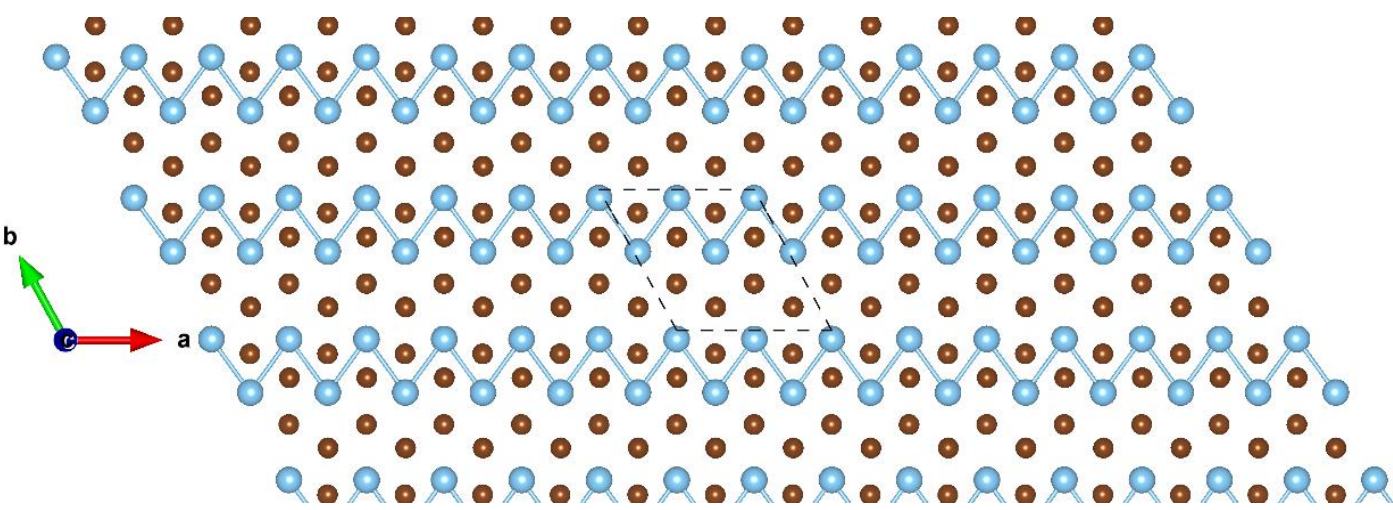

Figure S38: Top view of $\mathrm{TiBr}_{2} 2 \times 2 \phi_{-1}$. The sky blue and brown balls represent the $\mathrm{Ti}$ and $\mathrm{Br}$ atoms, respectively. 


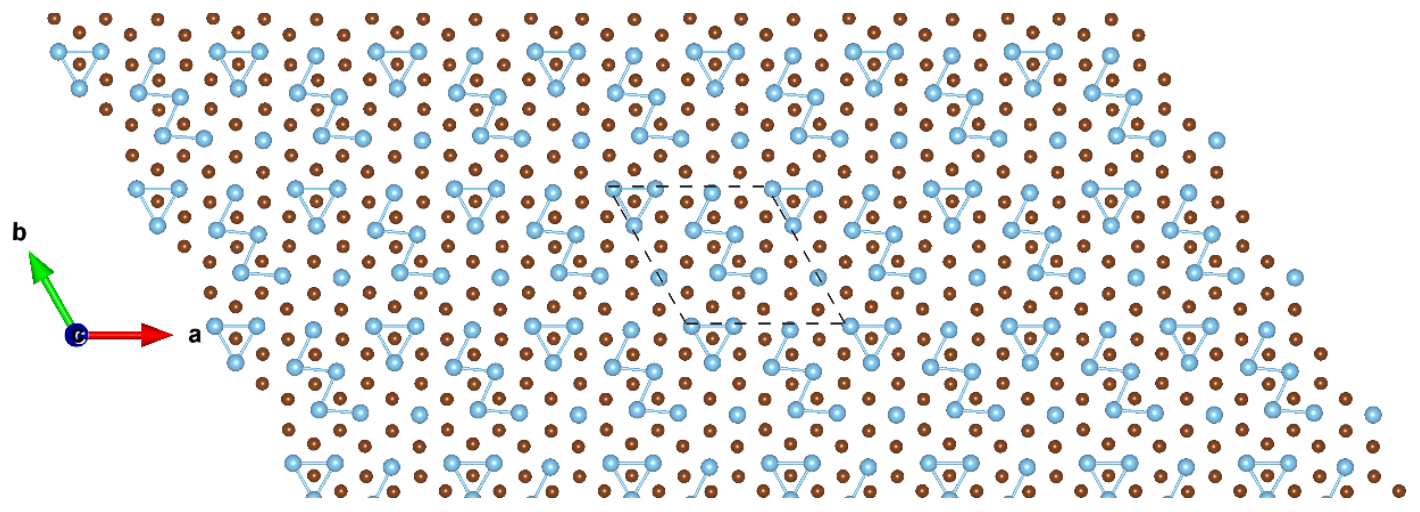

Figure S39: Top view of $\mathrm{TiBr}_{2} 3 \times 3 \phi_{-1}$.

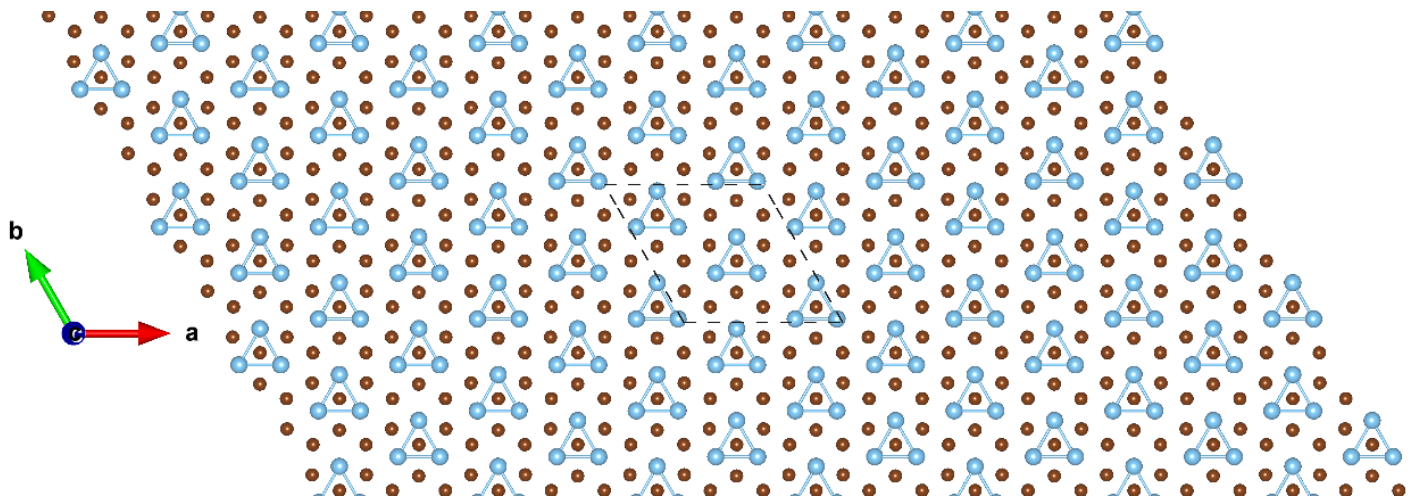

Figure S40: Top view of $\mathrm{TiBr}_{2} 3 \times 3 \phi_{-2}$.

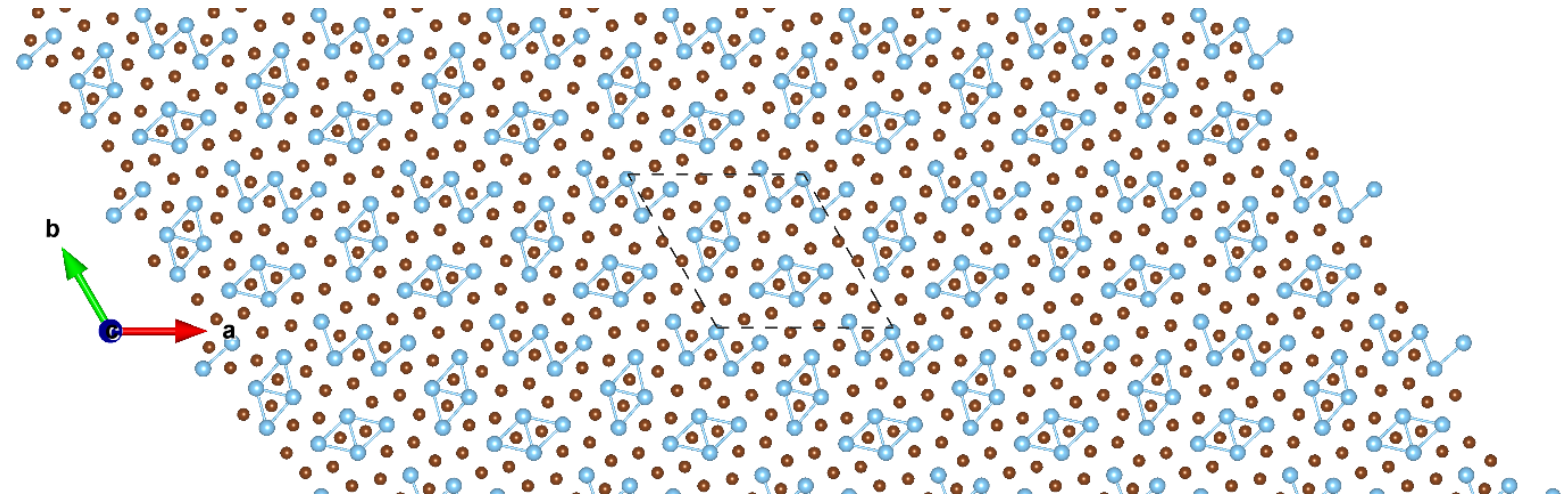

Figure S41: Top view of $\operatorname{TiBr}_{2} \sqrt{ } 13 \times \sqrt{ } 13 \phi-1$. 


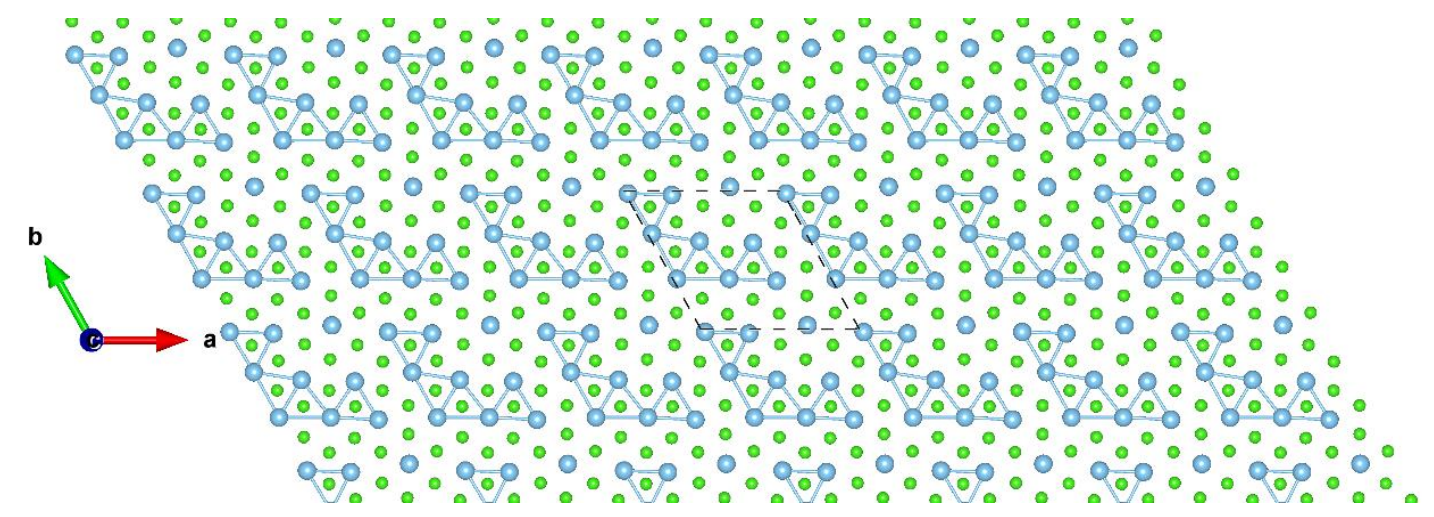

Figure S42: Top view of $\mathrm{TiCl}_{2} 3 \times 3 \phi_{-1}$. The sky blue and green balls represent the $\mathrm{Ti}$ and $\mathrm{Cl}$ atoms, respectively.

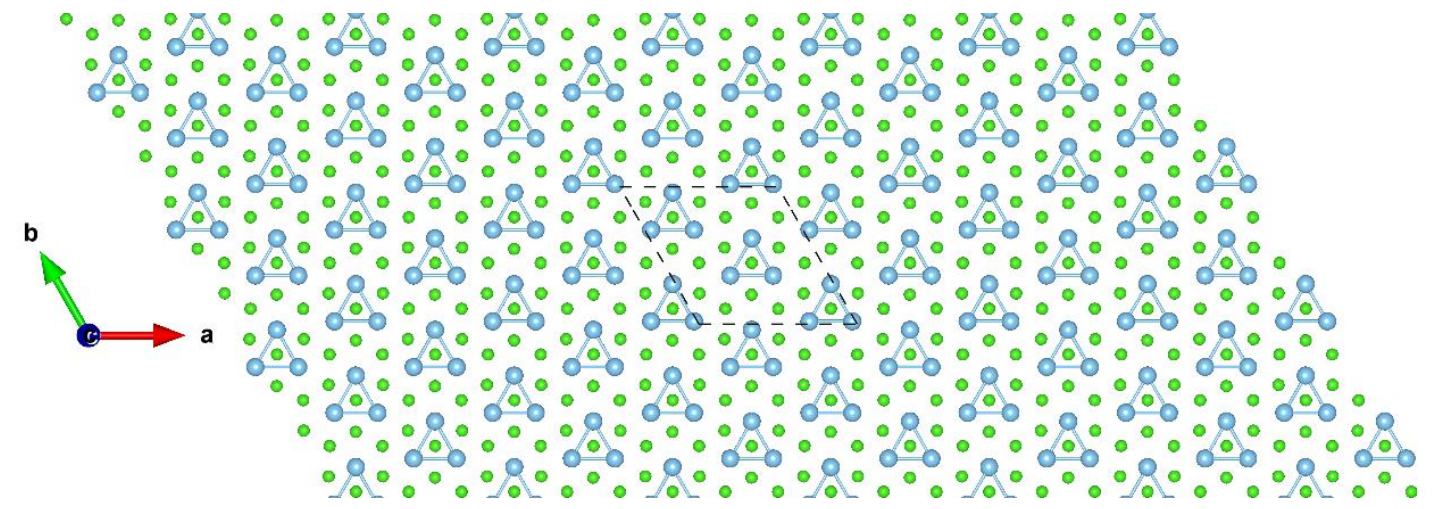

Figure S43: Top view of $\mathrm{TiCl}_{2} 3 \times 3 \phi_{-2}$. The sky blue and green balls represent the $\mathrm{Ti}$ and $\mathrm{Cl}$ atoms, respectively.

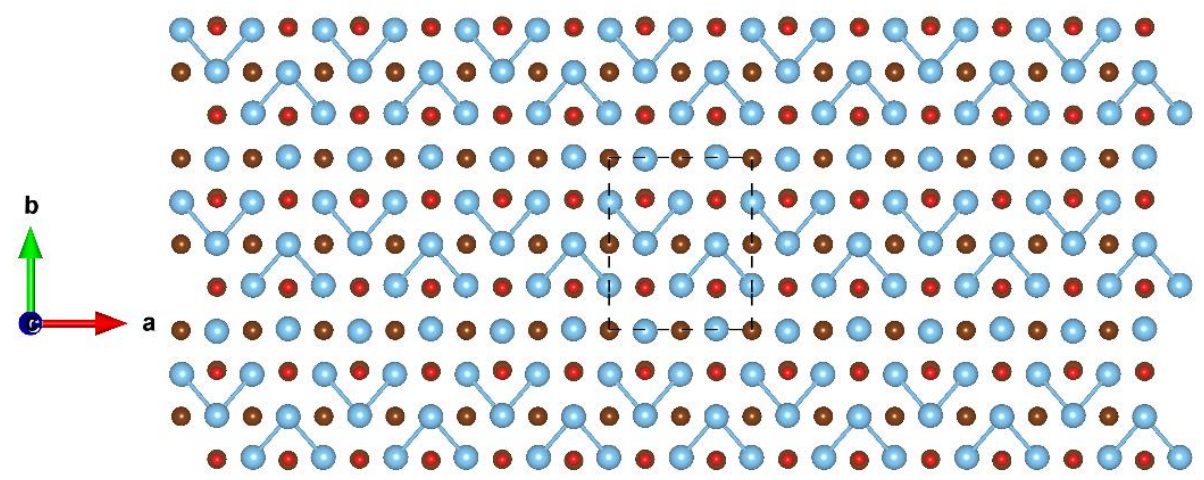

Figure S44: Top view of $\mathrm{TiOBr} 2 \times 2 \phi_{-1}$. The sky blue, red, and brown balls represent the $\mathrm{Ti}, \mathrm{O}$, and $\mathrm{Br}$ atoms, respectively. 


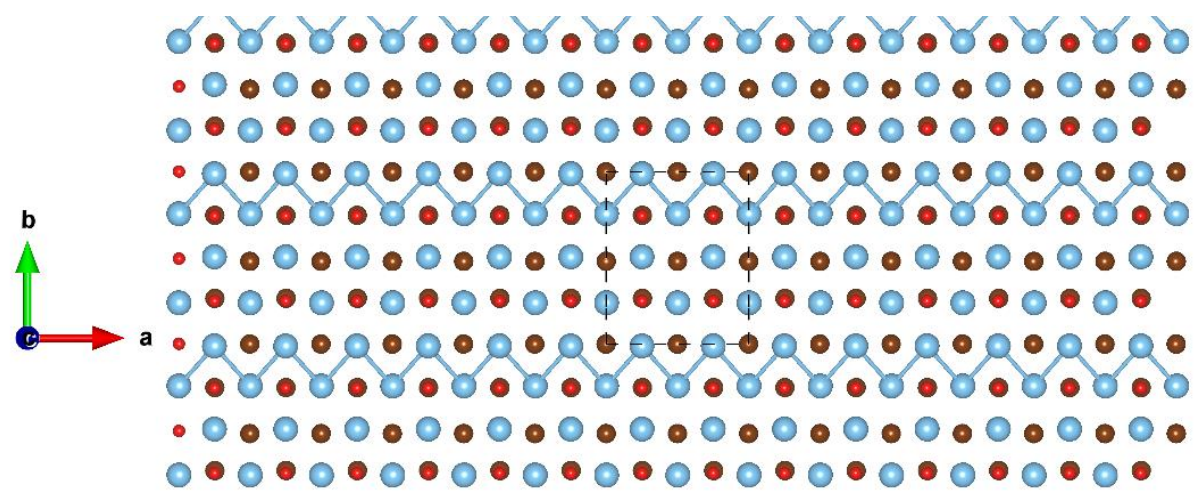

Figure S45: Top view of $\mathrm{TiOBr} 2 \times 2 \phi_{-2}$.

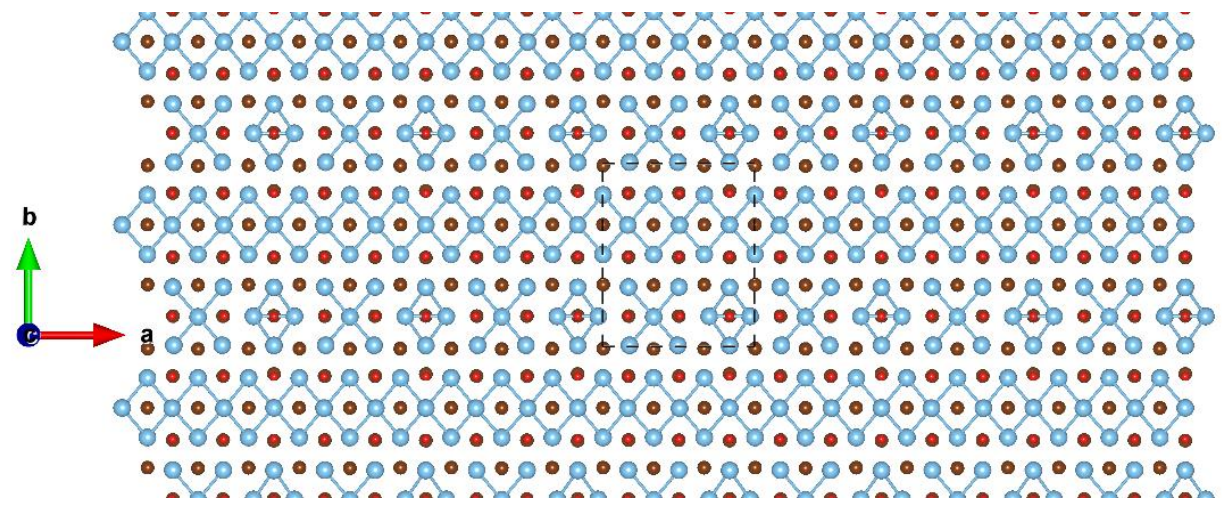

Figure S46: Top view of $\mathrm{TiOBr} 3 \times 3 \phi_{-1}$.

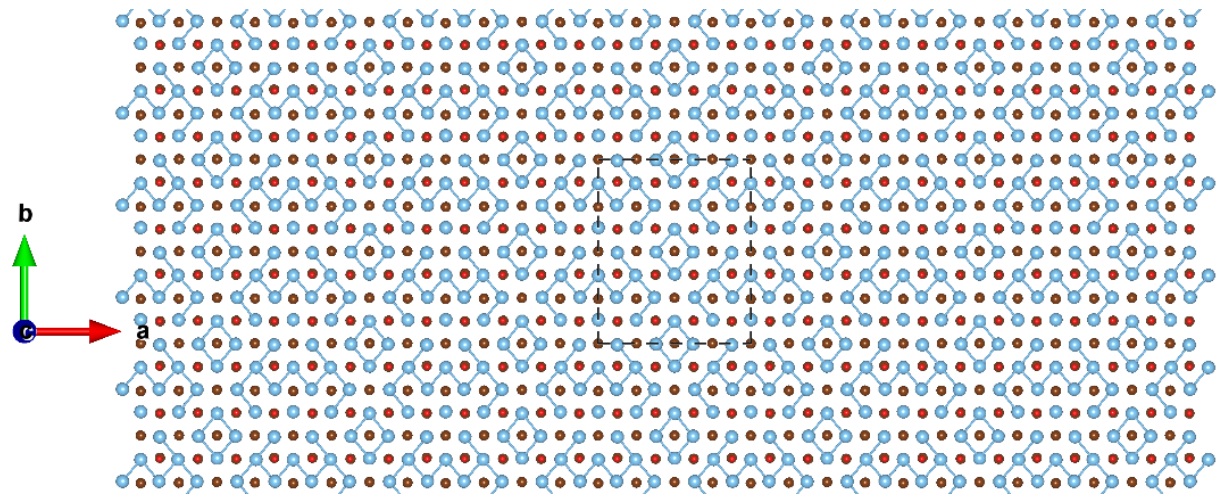

Figure S47: Top view of $\mathrm{TiOBr} 4 \times 4 \phi_{-1}$. 


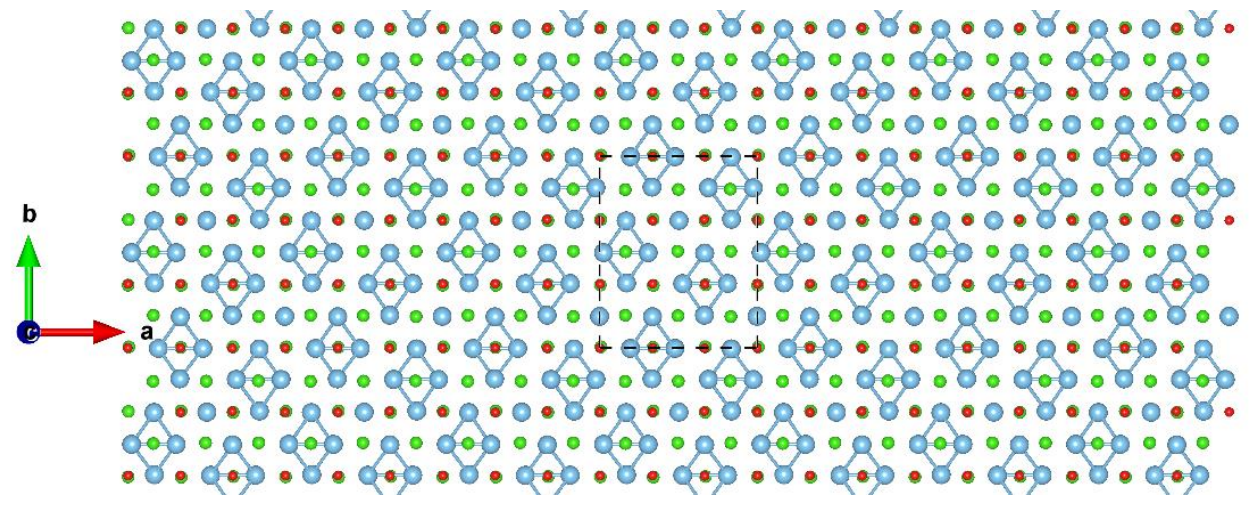

Figure S48: Top view of $\mathrm{TiOCl} 3 \times 3 \phi_{-1}$. The sky blue, red, and green balls represent the Ti, O, and $\mathrm{Cl}$ atoms, respectively.

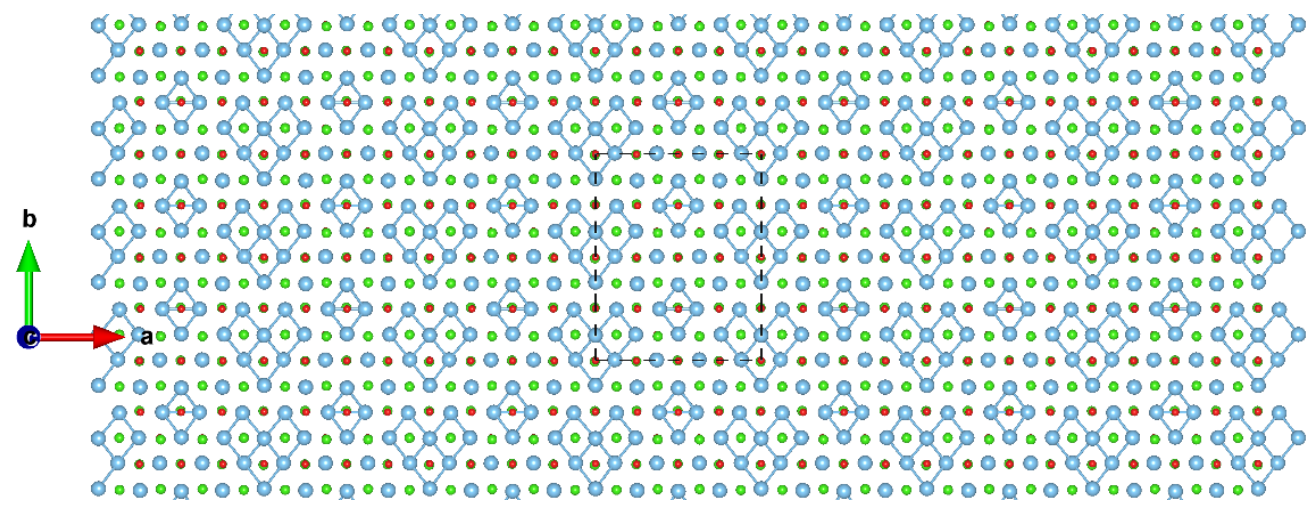

Figure S49: Top view of TiOCl $4 \times 4 \phi_{-1}$.

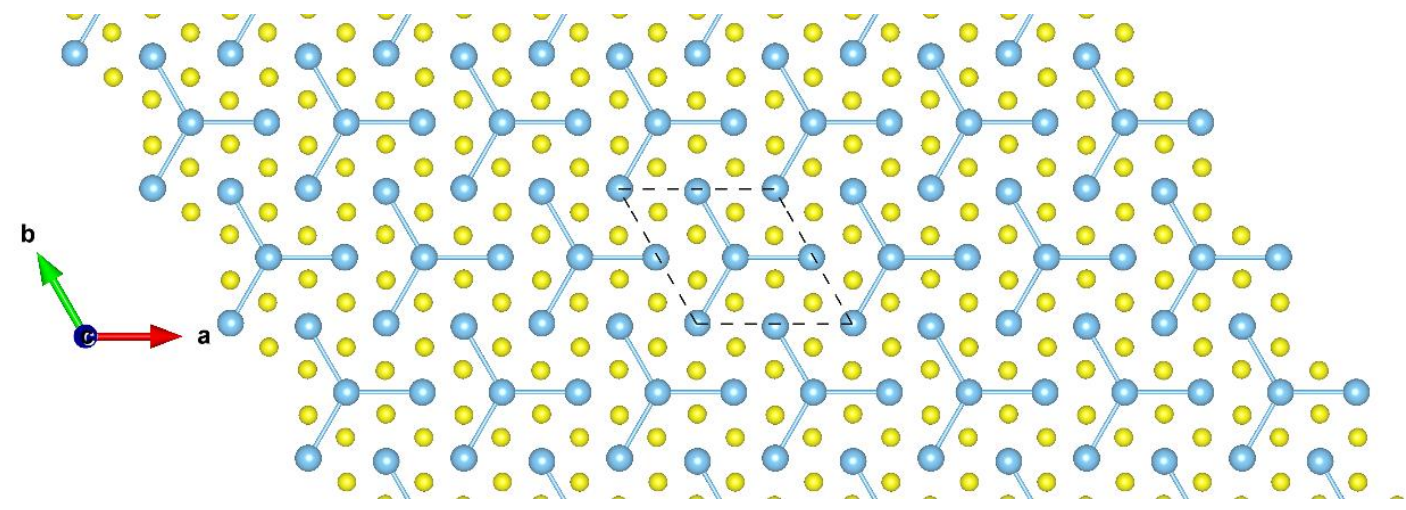

Figure S50: Top view of $\mathrm{TiS}_{2} 2 \times 2 \phi_{-1}$. The sky blue and yellow balls represent the $\mathrm{Ti}$ and $\mathrm{S}$ atoms, respectively. 


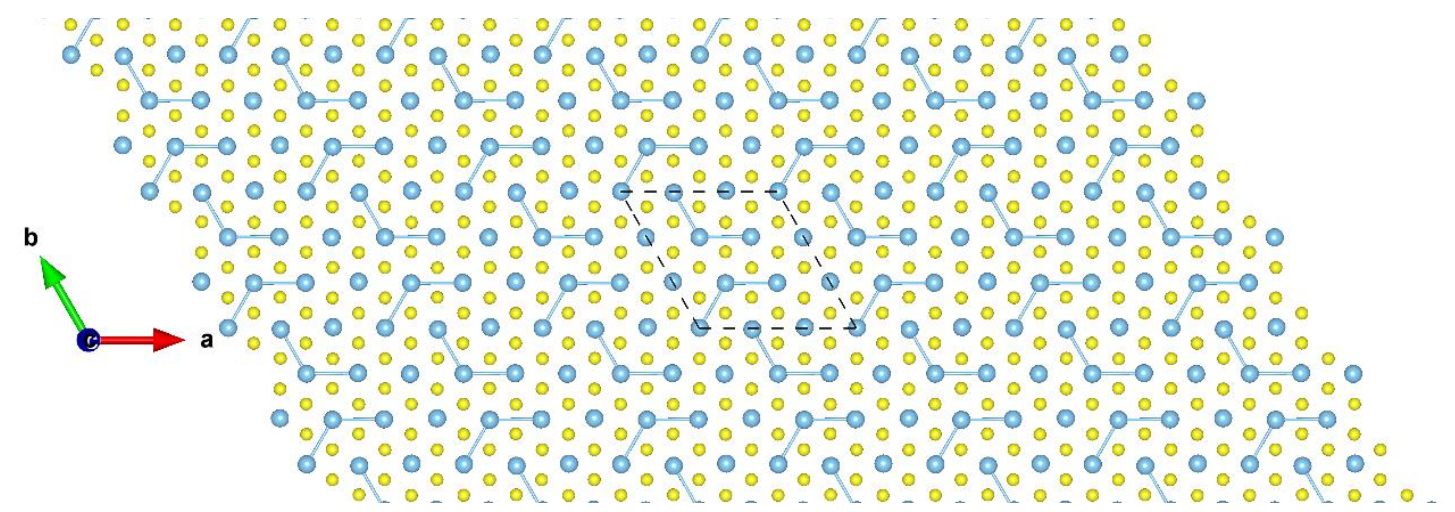

Figure S51: Top view of $\mathrm{TiS}_{2} 3 \times 3 \phi_{-1}$.

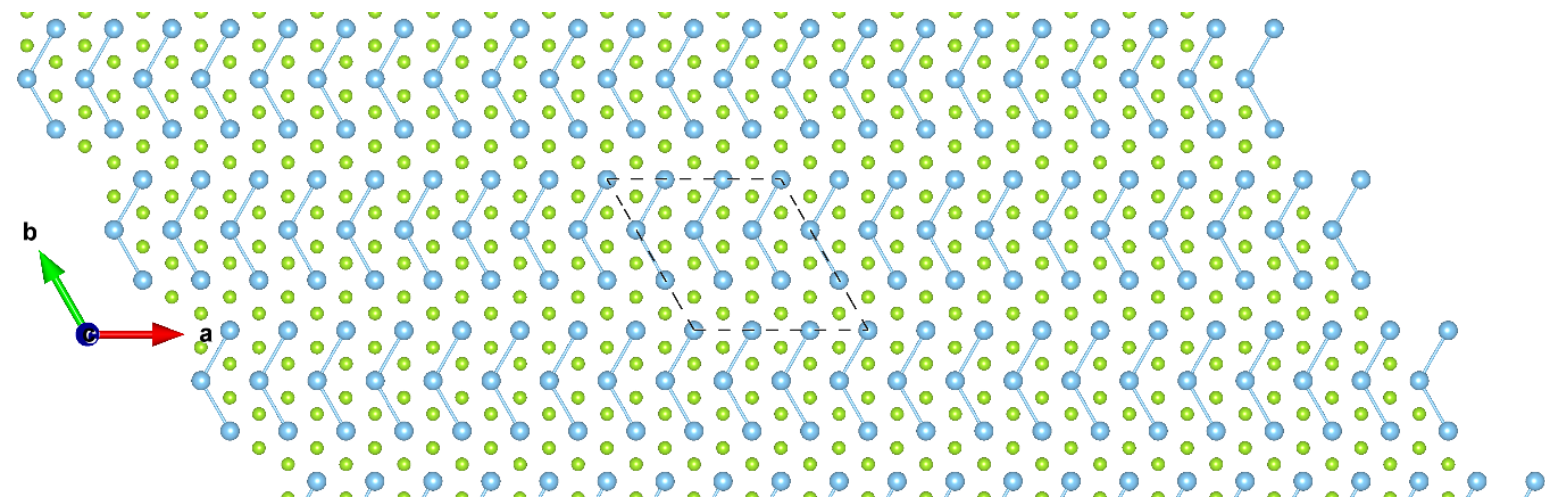

Figure S52: Top view of $\mathrm{TiSe}_{2} 3 \times 3 \phi-1$. The sky blue and light green balls represent the $\mathrm{Ti}$ and $\mathrm{Se}$ atoms, respectively.

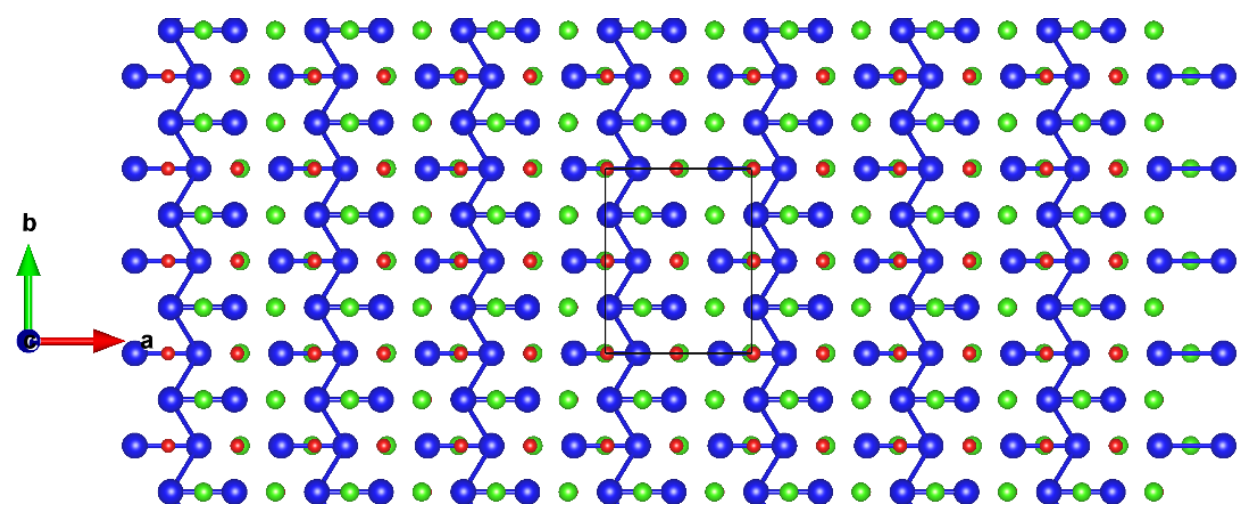

Figure S53: Top view of $\operatorname{VOCl} 2 \times 2 \phi_{-1}$. The royal blue, red, and light green balls represent the V, $\mathrm{O}$, and $\mathrm{Cl}$ atoms, respectively. 


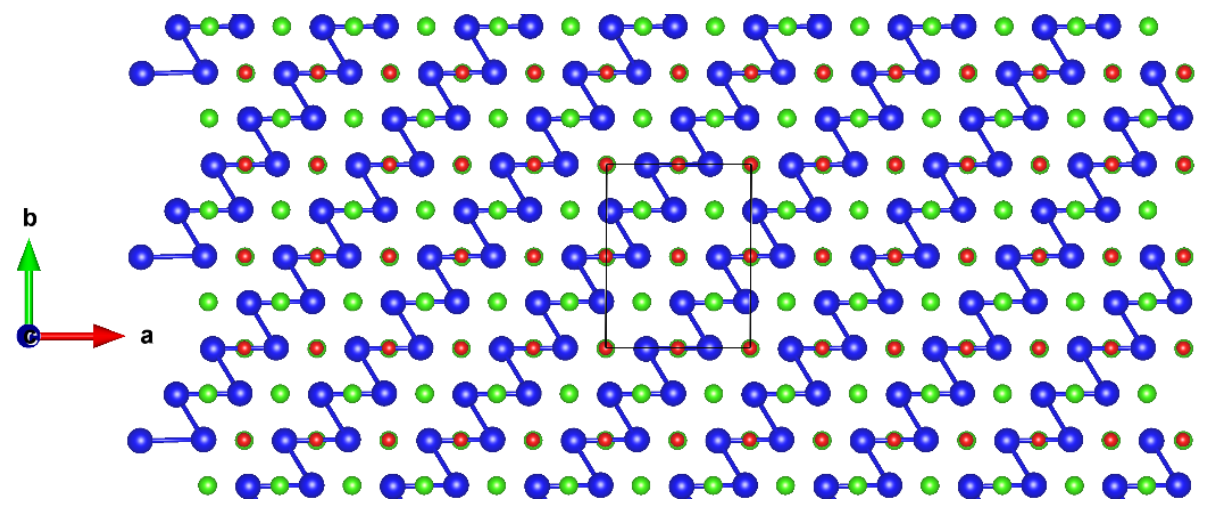

Figure S54: Top view of $\mathrm{VOCl} 2 \times 2 \phi_{-2}$.

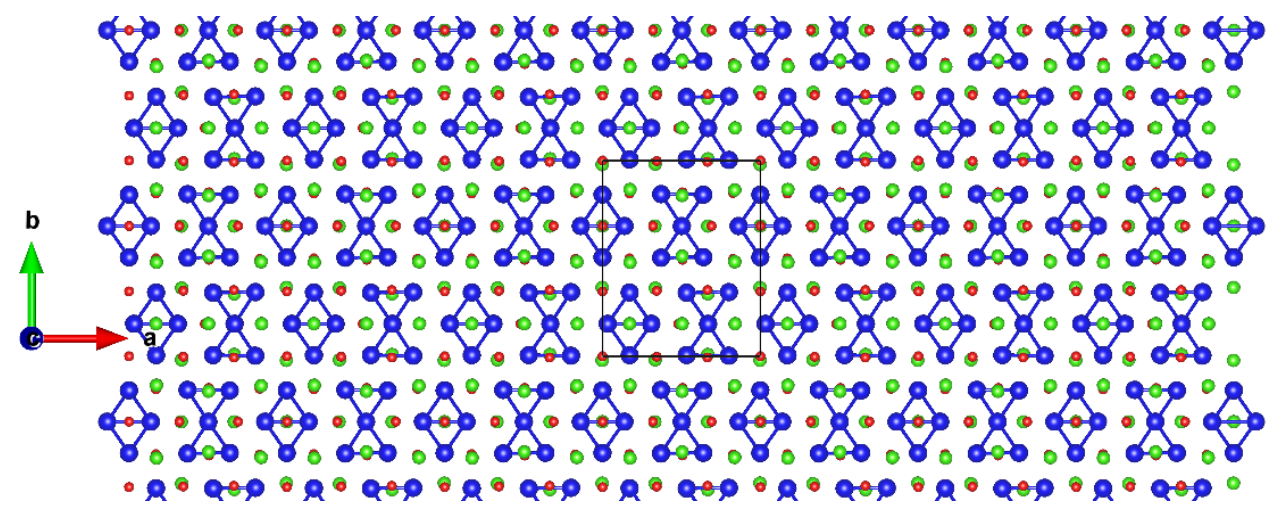

Figure S55: Top view of $\mathrm{VOCl} 3 \times 3 \phi_{-1}$.

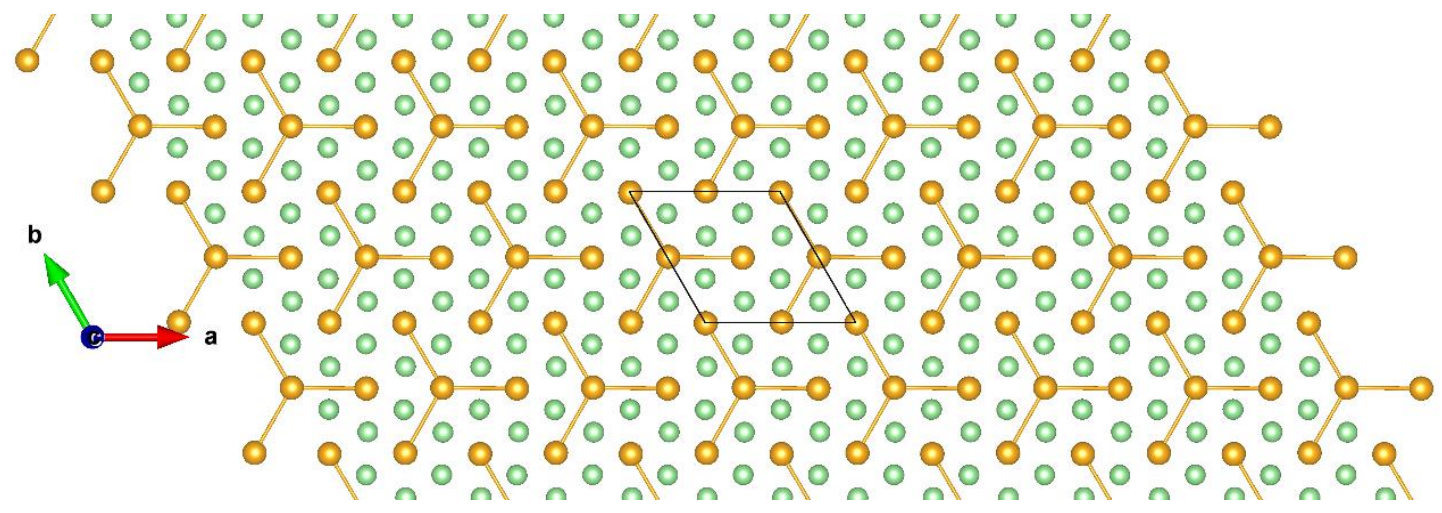

Figure S56: Top view of $\mathrm{ZrTe}_{2} 2 \times 2 \phi-1$. The orange and green balls represent the $\mathrm{Zr}$ and Te atoms, respectively. 


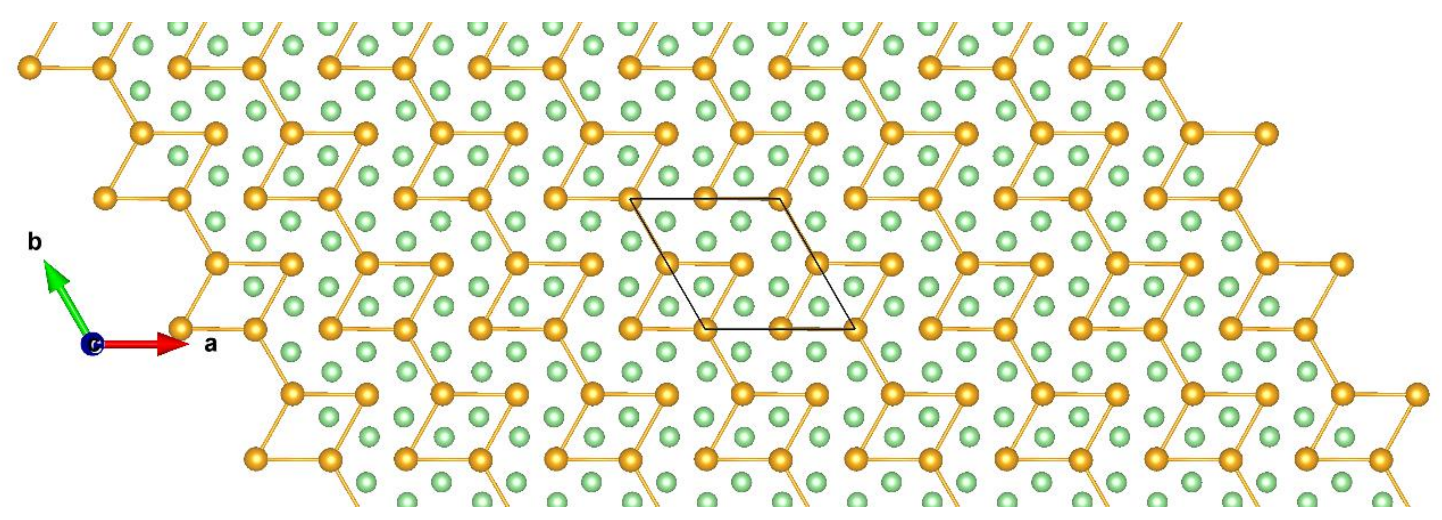

Figure S57: Top view of $\mathrm{ZrTe}_{2} 2 \times 2 \phi-2$.

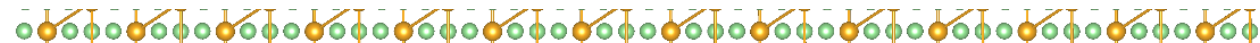
00000000000000000000000000000000000000000000000000000000

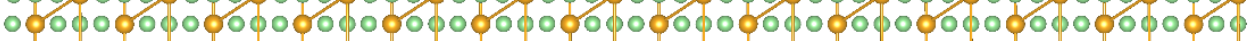
00000000000000000000000000000000000000000000000000000000 00000000000000000000000000000000000000000000000000000000 00000000000000000000000000000000000000000000000000000000 b $000000000000000000000000 \$ 0000000000000000000000000000000$ 100000000000000000000000000000000000000000000000000000000 000\$0000000000000000000100000000000000010000000000000000 00000000000000000000000000000000000000000000000000000000

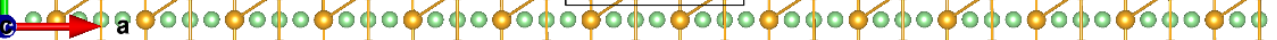
00000000000000000000000000000000000000000000000000000000 00000000000000000000000000000000000000000000000000000000 00000000000000000000000000000000000000000000000000000000 00000000000000000000000000000000000000000000000000000

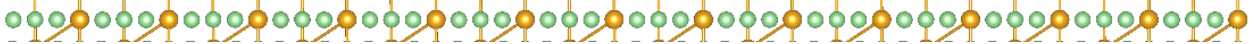

Figure S58: Top view of $\mathrm{ZrTe}_{3} 2 \times 2 \phi_{-1}$. The same color convention of the last 2 Figures has been used.

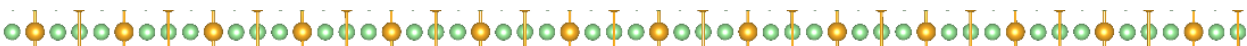
00000000000000000000000000000000000000000000000000000000 00000000000000000000000000000000000000000000000000000000 00000000000000000000000000000000000000000000000000000000 $0000000 \$ 0000000 \$ 000000000000000000000000000000000000000 \phi$ 00000000000000000000000000000000000000000000000000000000 b 00000000000000000000000050000000000000000000000000000000 00000000000000000000000000000000000000000000000000000000 $000 \$ 000000000000000 \phi 000 \phi 00000000000000000000000000000000$ 00000000000000000000000000000000000000000000000000000000 nan a 000000000000000000000000000000000000000000000000000 00000000000000000000000000000000000000000000000000000000 $0000000000000000000000000000000000000000000000000000000 \phi$ 00000000000000000000000000000000000000000000000000000000 $0000000 \phi 000000000000000000000000000000000000000000000000$ 00000000000000000000000000000000000000000000000000000000

Figure S59: Top view of $\mathrm{ZrTe}_{3} 2 \times 2 \phi_{-2}$. 


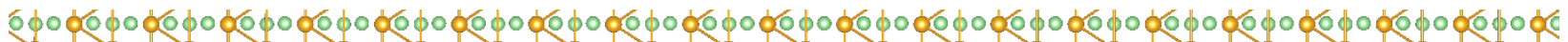
000000000000000000000000000000000000000000000000000000000000000000000000000000000

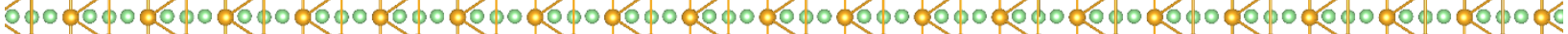
000000000000000000000000000000000000000000000000000000000000000000000000000001

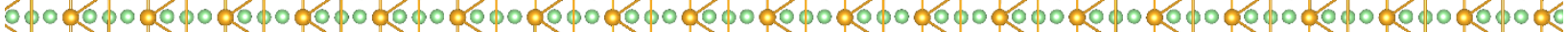
$00000000000000000000000000000000000000000000000000000000000000000000000000000000 c$

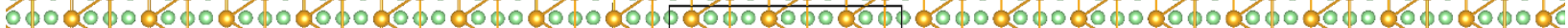
000000 010000000000000000000000000000000000000000000000000000000000000000000000c

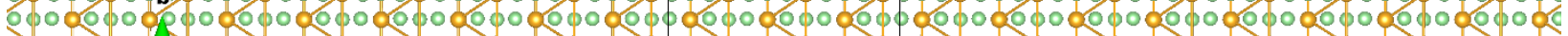
00000002000000000000000000000000000000000000000000000000000000000000000000000000

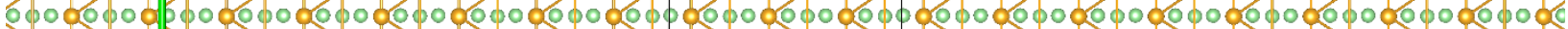
$000000090000000000000000000000000000000000000000000000000000000000000000000000 c$

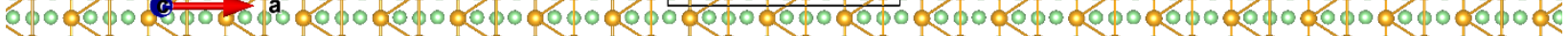
000000000000000000000000000000000000000000000000000000000000000000000000000000000 0 0000000000000000000000000000000000000000000000000000000000000000000000000000001 00000000000000000000000000000000000000000000000000000000000000000000000000000000 c 0000000000000000010000000000000000000000000000000000000000000000000000000000000 000000000000000000000000000000000000000000000000000000000000000000000000000000000

Figure S60: Top view of $\mathrm{ZrTe}_{3} 3 \times 3 \phi_{-1}$.

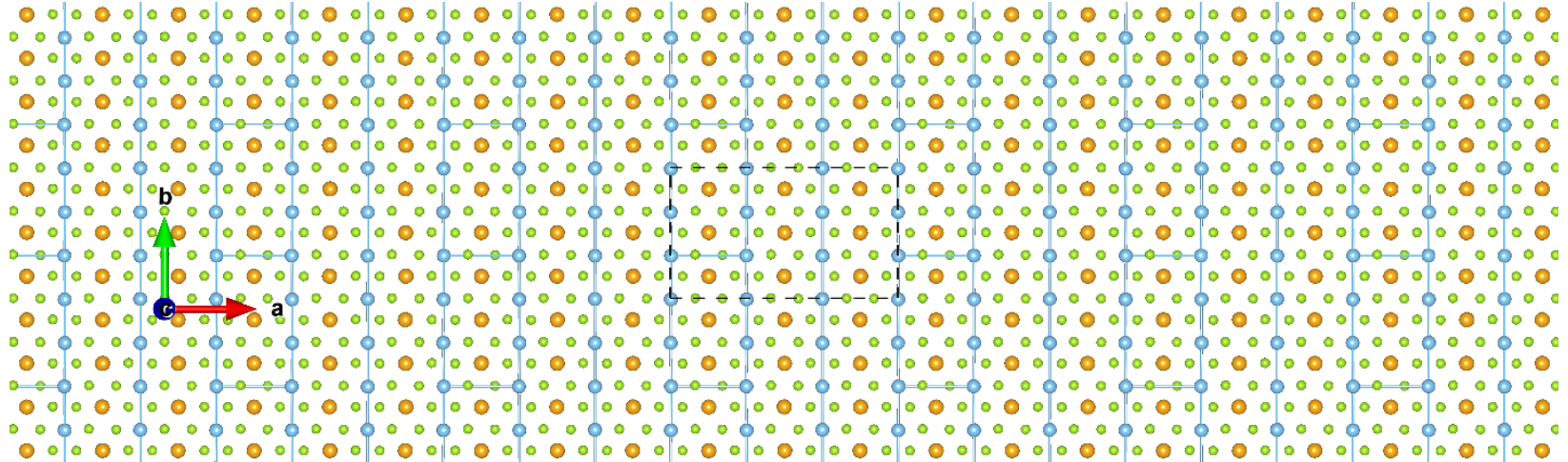

Figure S61: Top view of $\mathrm{ZrTiSe}_{4} 3 \times 3 \phi_{-1}$. The orange, blue, and green balls represent the $\mathrm{Zr}$, Ti and Se atoms, respectively.

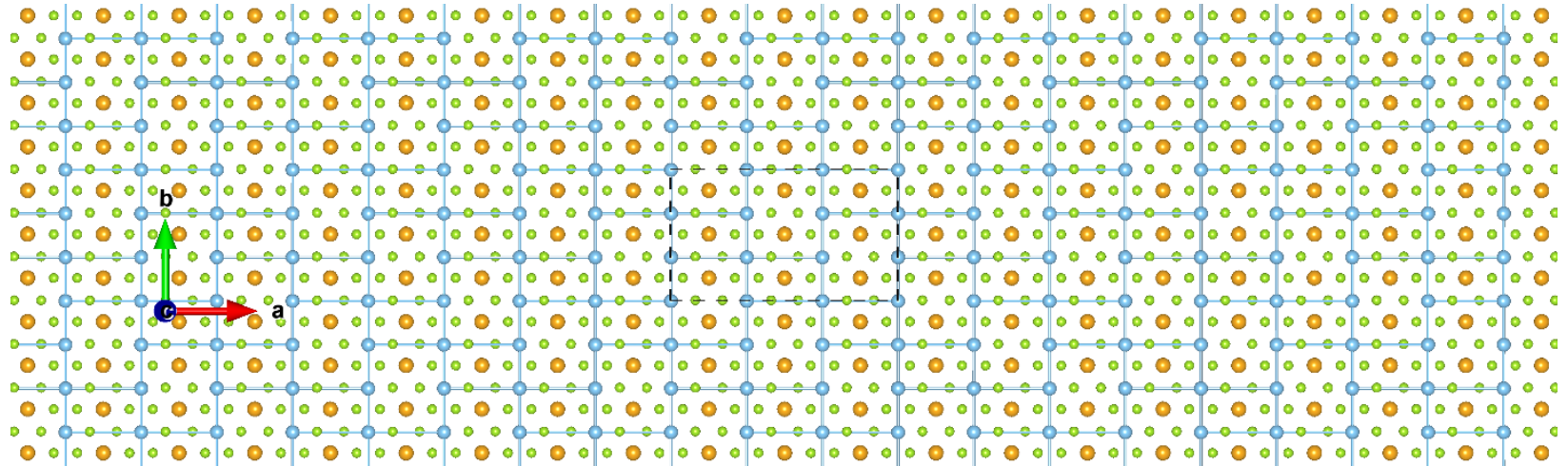

Figure S62: Top view of $\mathrm{ZrTiSe}_{4} 3 \times 3 \phi_{-2}$. 


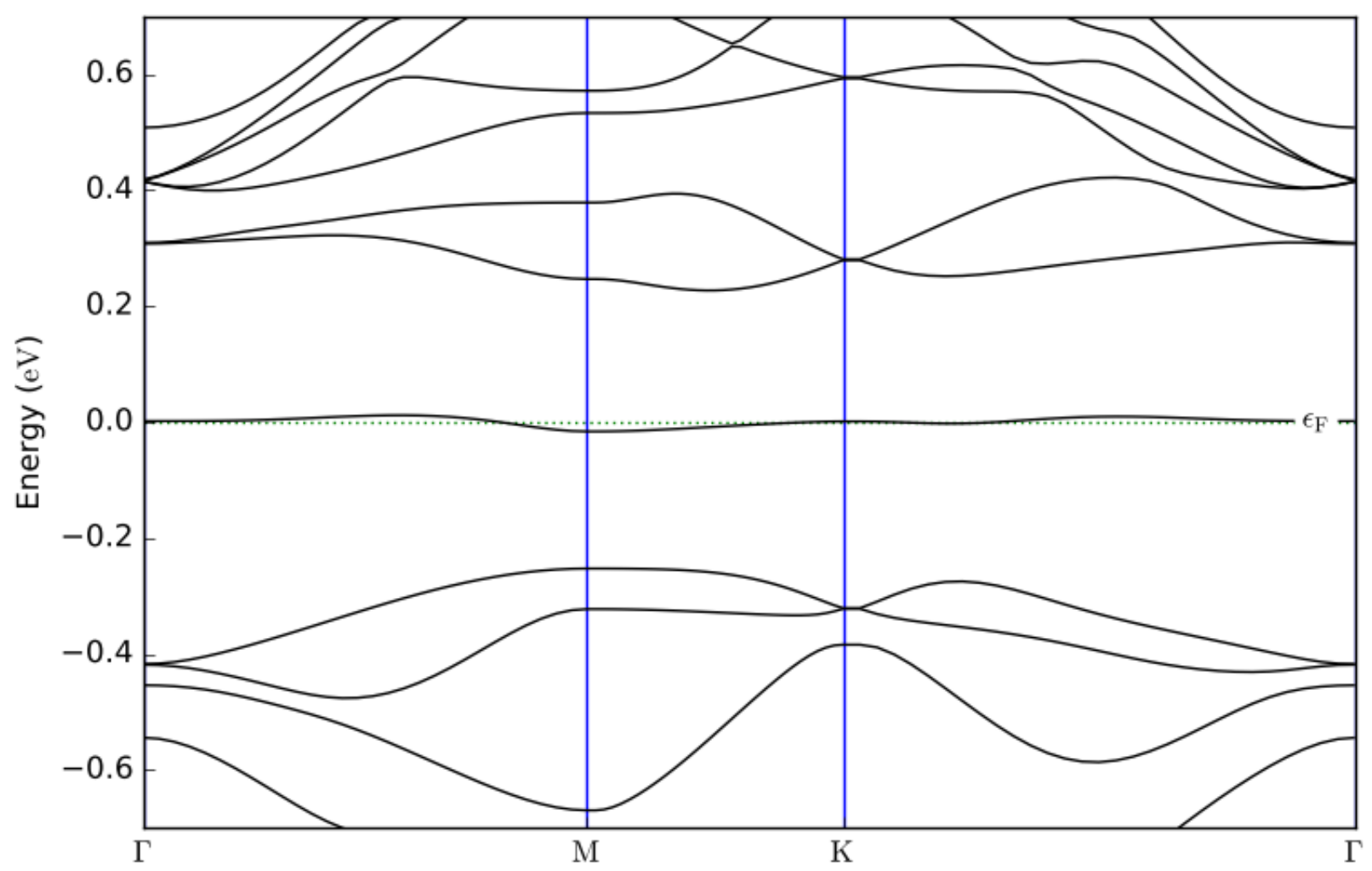

Figure S63: Band structure of $1 \mathrm{~T}-\mathrm{TaS}_{2} \sqrt{ } 13 \times \sqrt{ } 13 \phi_{-2}(\mathrm{CCDW})$. Note that for this and all subsequent band structures, the supercell band structure is presented and thus, the effect of BZ folding is included. 


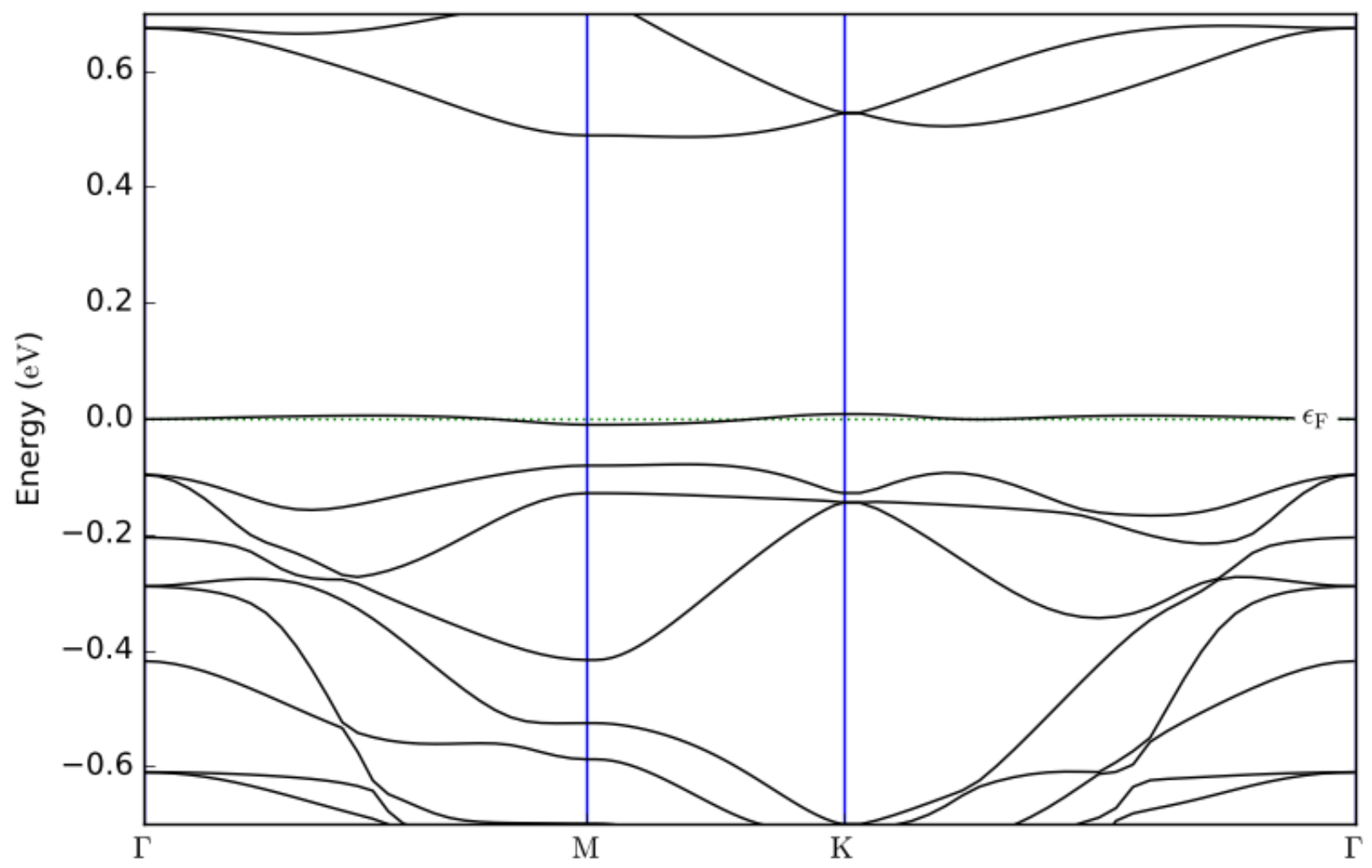

Figure S64: Band structure of 1T-TaSe $2 \sqrt{13} \times \sqrt{ } 13 \phi_{-1}$. 


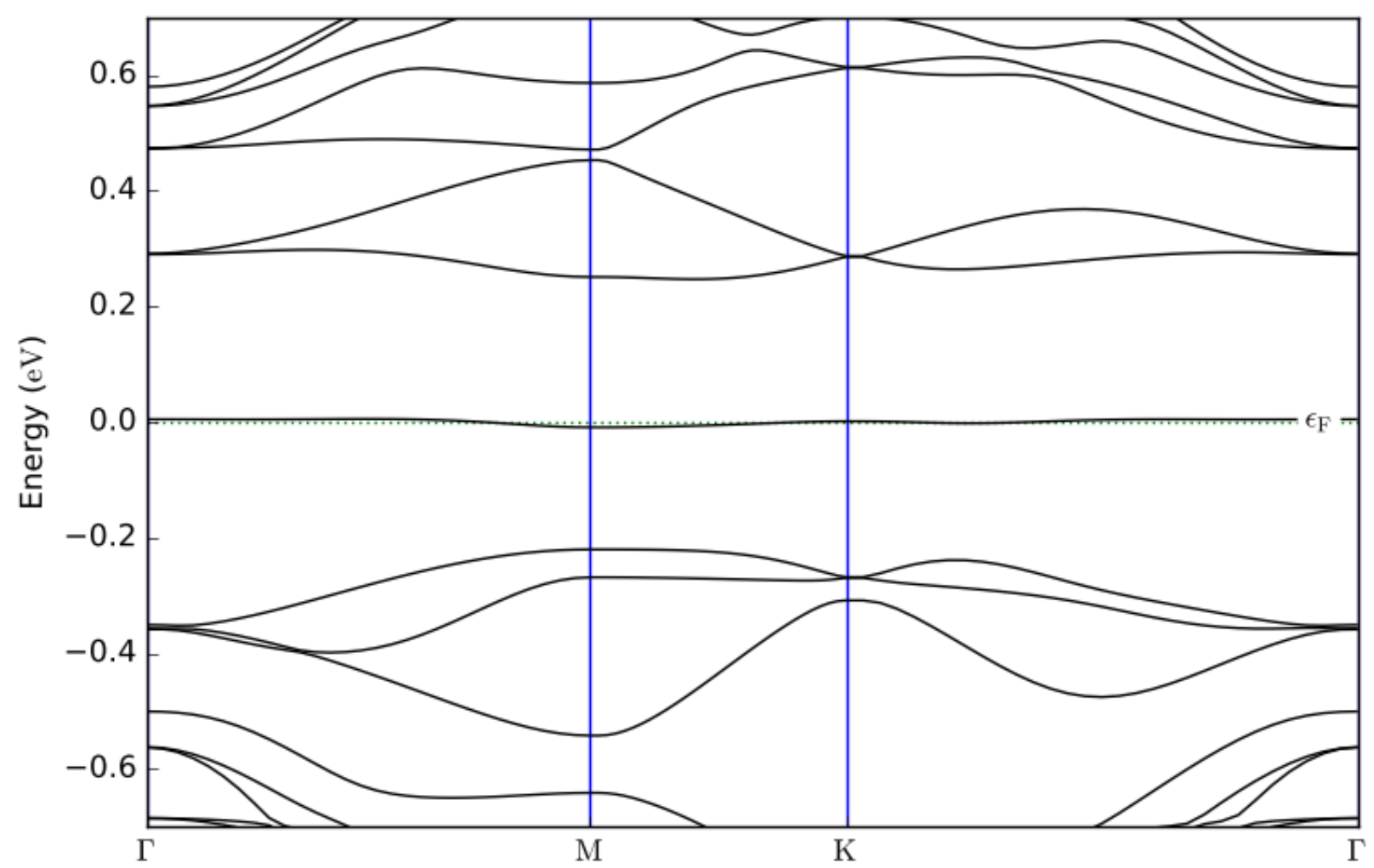

Figure S65: Band structure of 1T-NbSe ${ }_{2} \sqrt{13} \times \sqrt{13} \phi_{-1}$. 


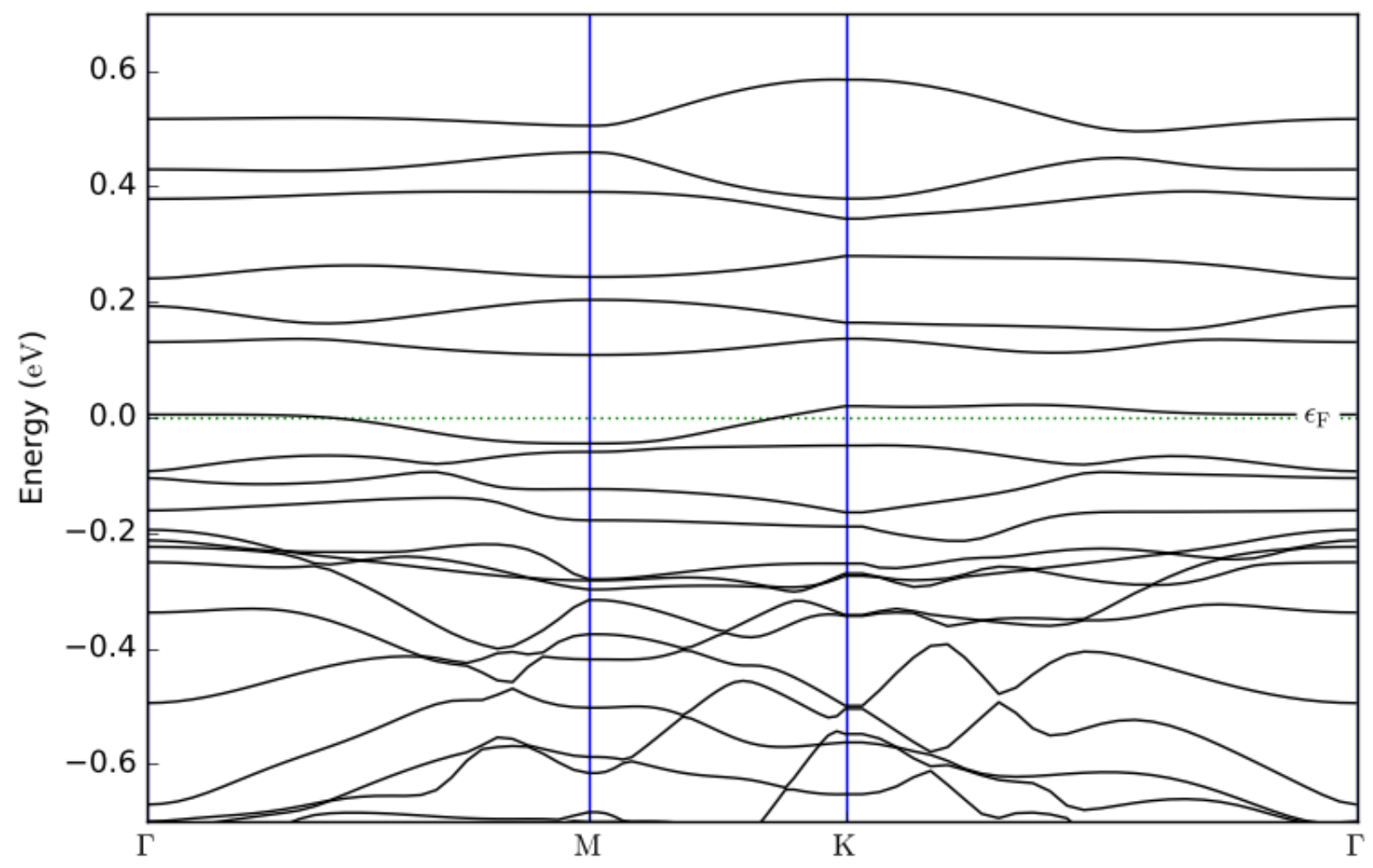

Figure S66: Band structure of $2 \mathrm{H}-\mathrm{NbTe}_{2} \sqrt{13} \times \sqrt{13} \phi_{-1}$. 


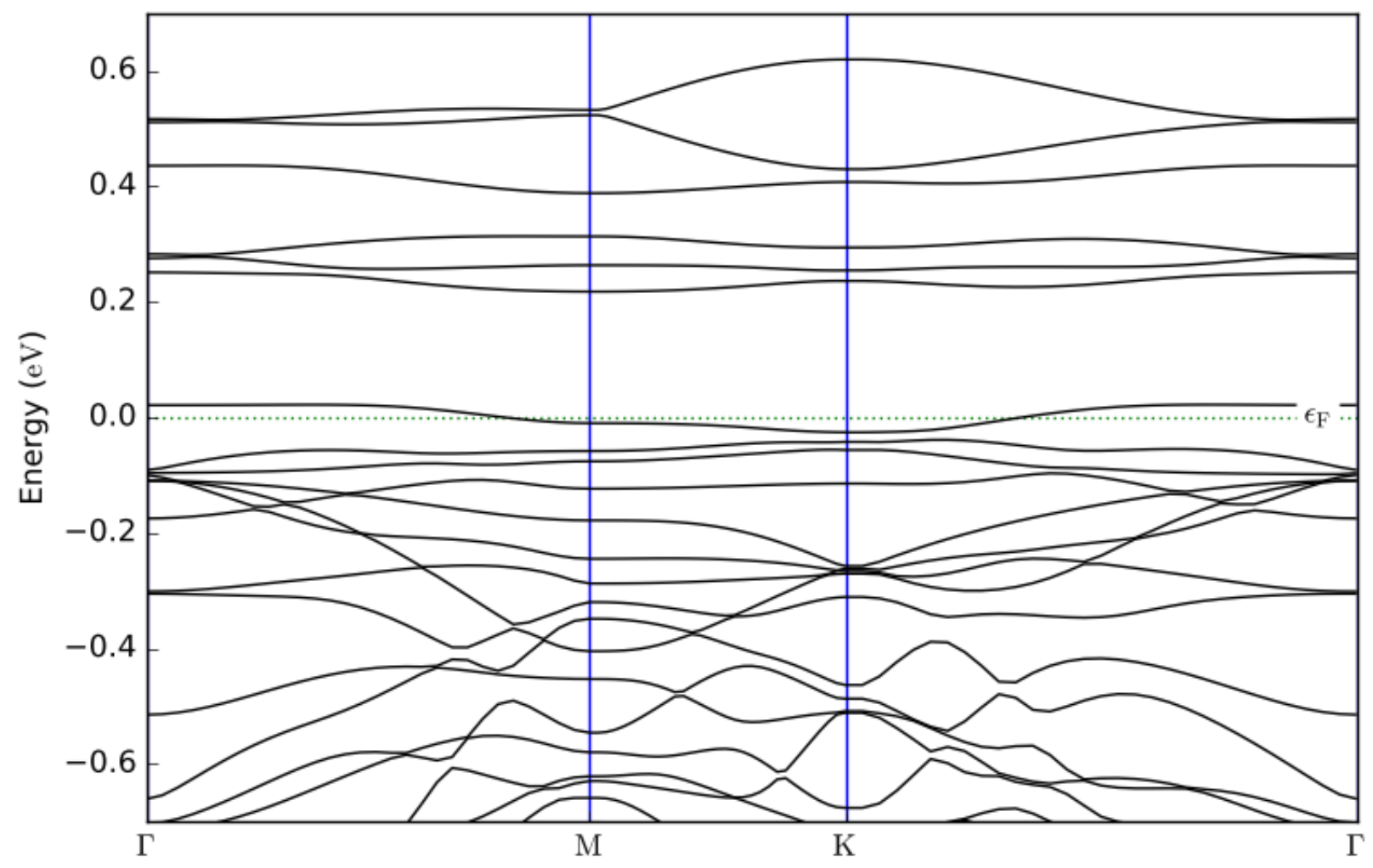

Figure S67: Band structure of $2 \mathrm{H}-\mathrm{NbTe}_{2} \sqrt{13} \times \sqrt{13} \phi_{-2}$. 


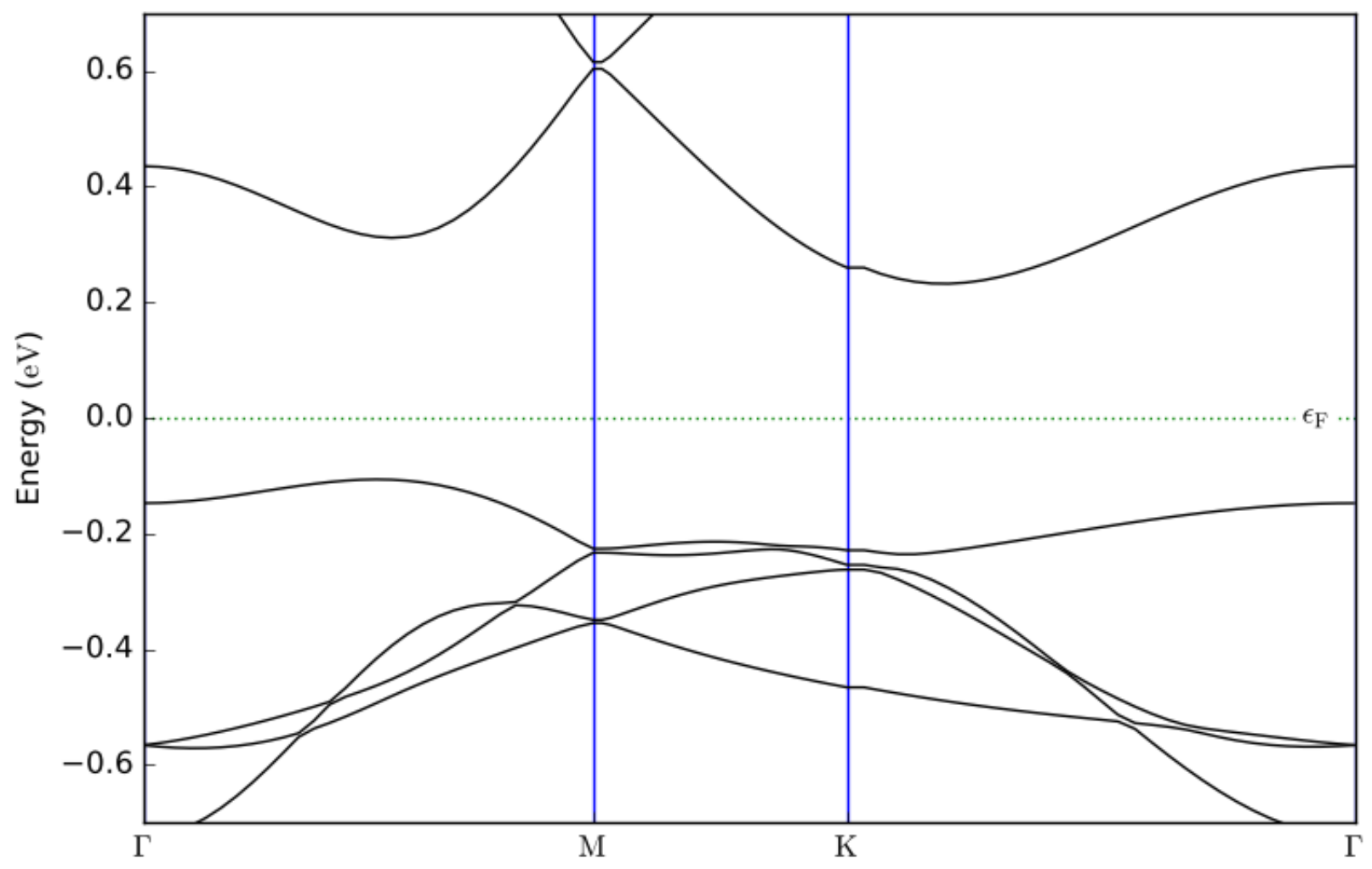

Figure S68: Band structure of $\mathrm{TiBr}_{2} 2 \times 2 \phi_{-1}$. 


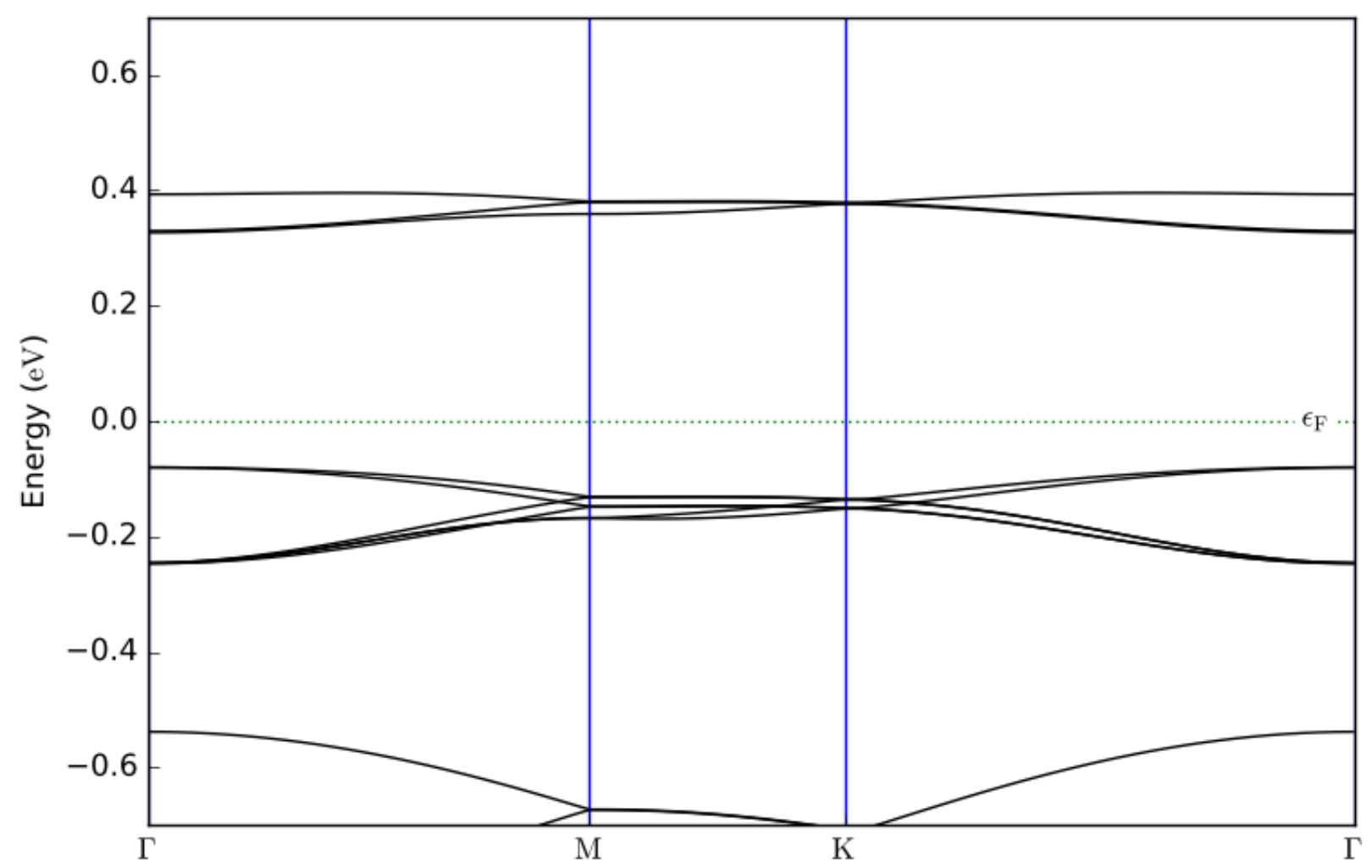

Figure S69: Band structure of $\mathrm{TiBr}_{2} 3 \times 3 \phi_{-2}$. 


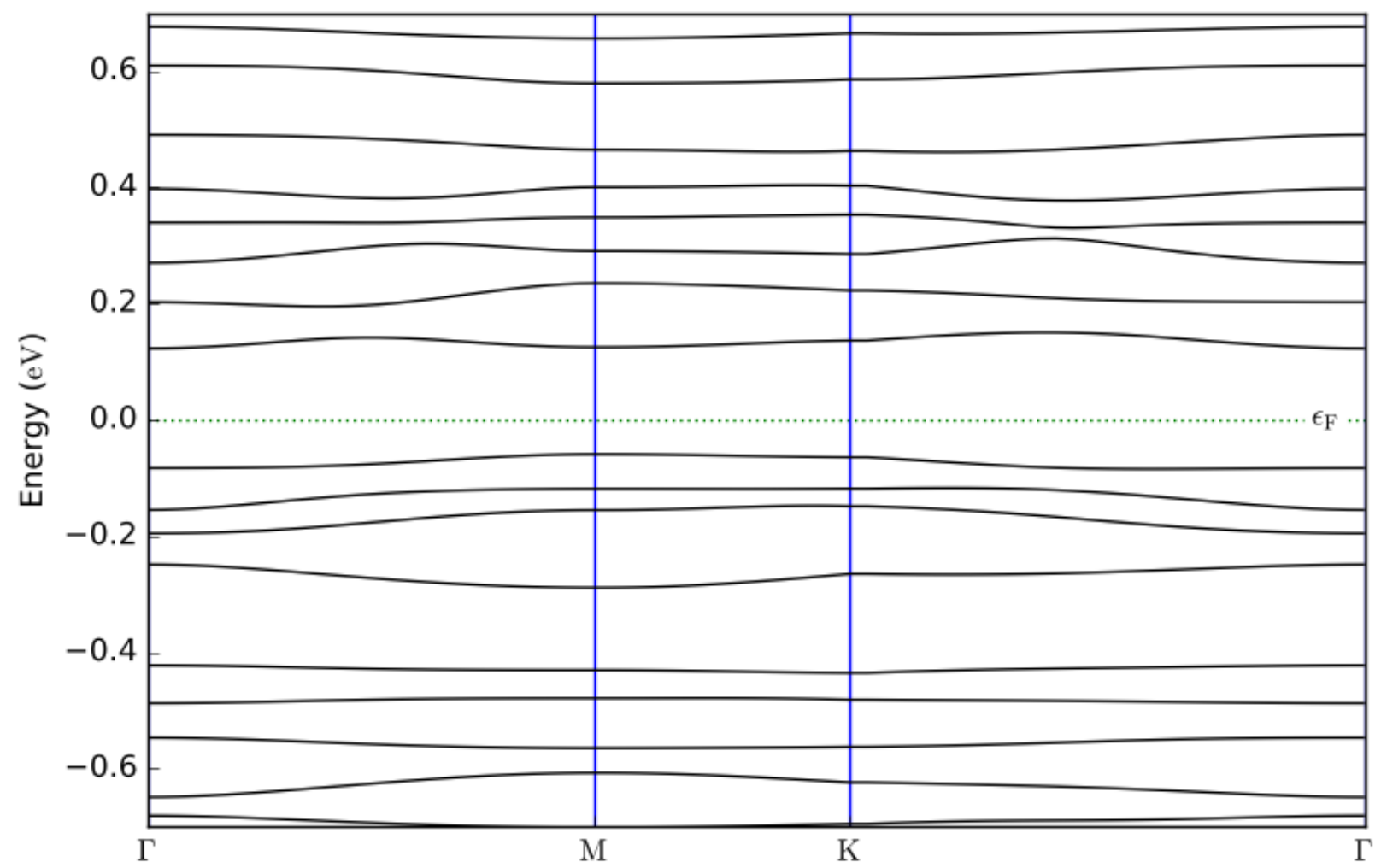

Figure S70: Band structure of $\mathrm{TiBr}_{2} \sqrt{ } 13 \times \sqrt{ } 13 \phi_{-1}$. 


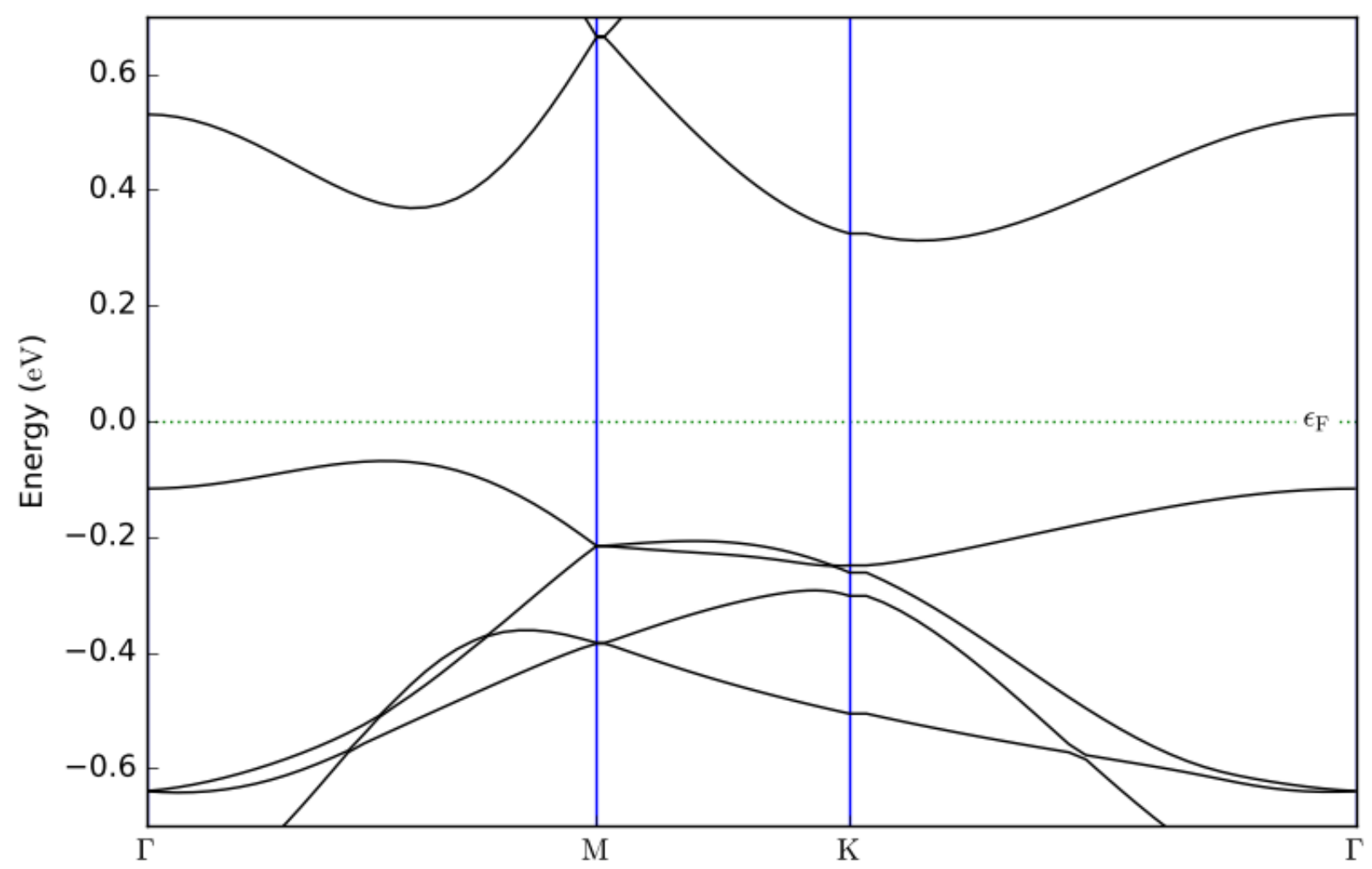

Figure S71: Band structure of $\mathrm{TiCl}_{2} 2 \times 2 \phi_{-1}$. 


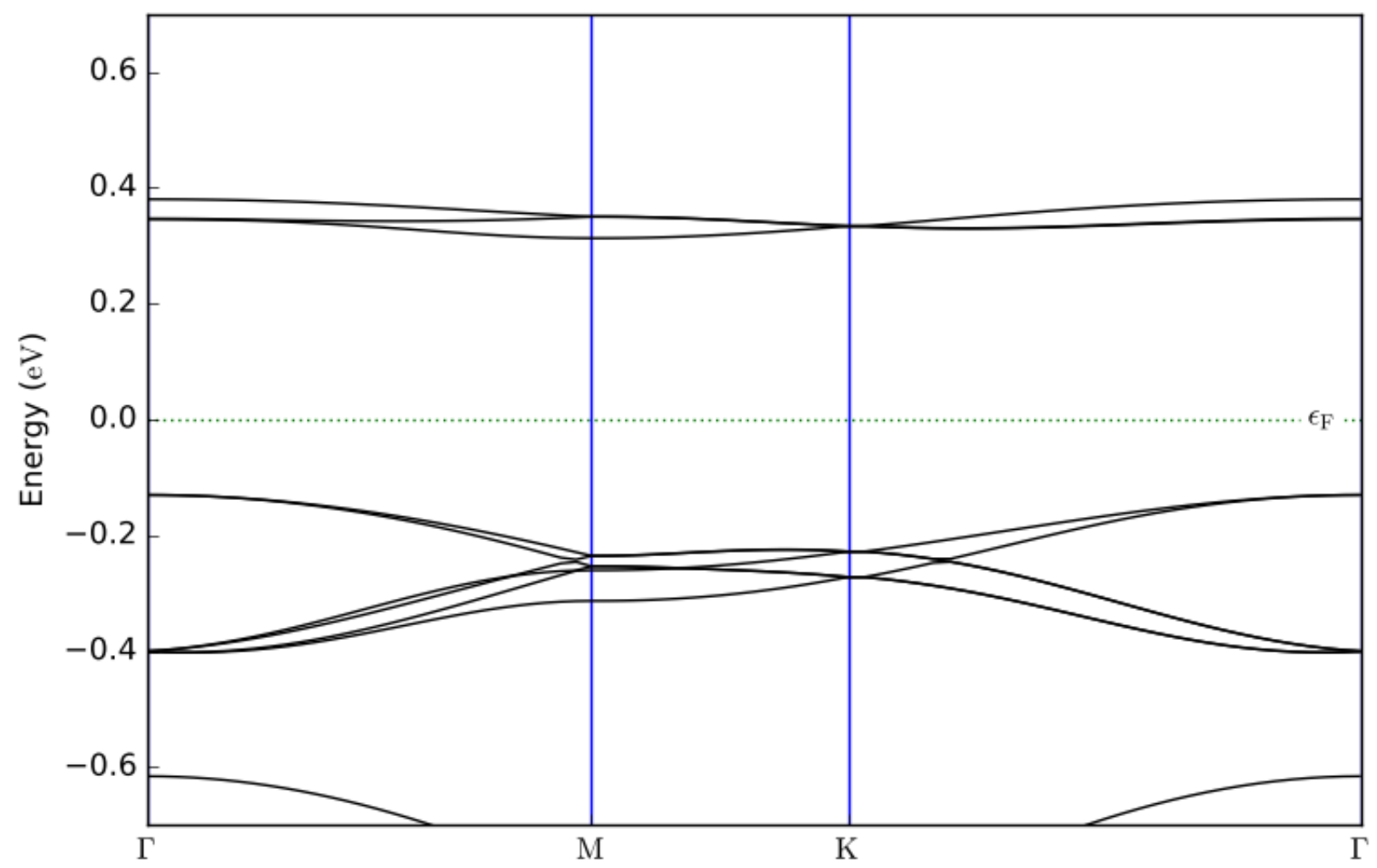

Figure S72: Band structure of $\mathrm{TiCl}_{2} 3 \times 3 \phi_{-2}$. 


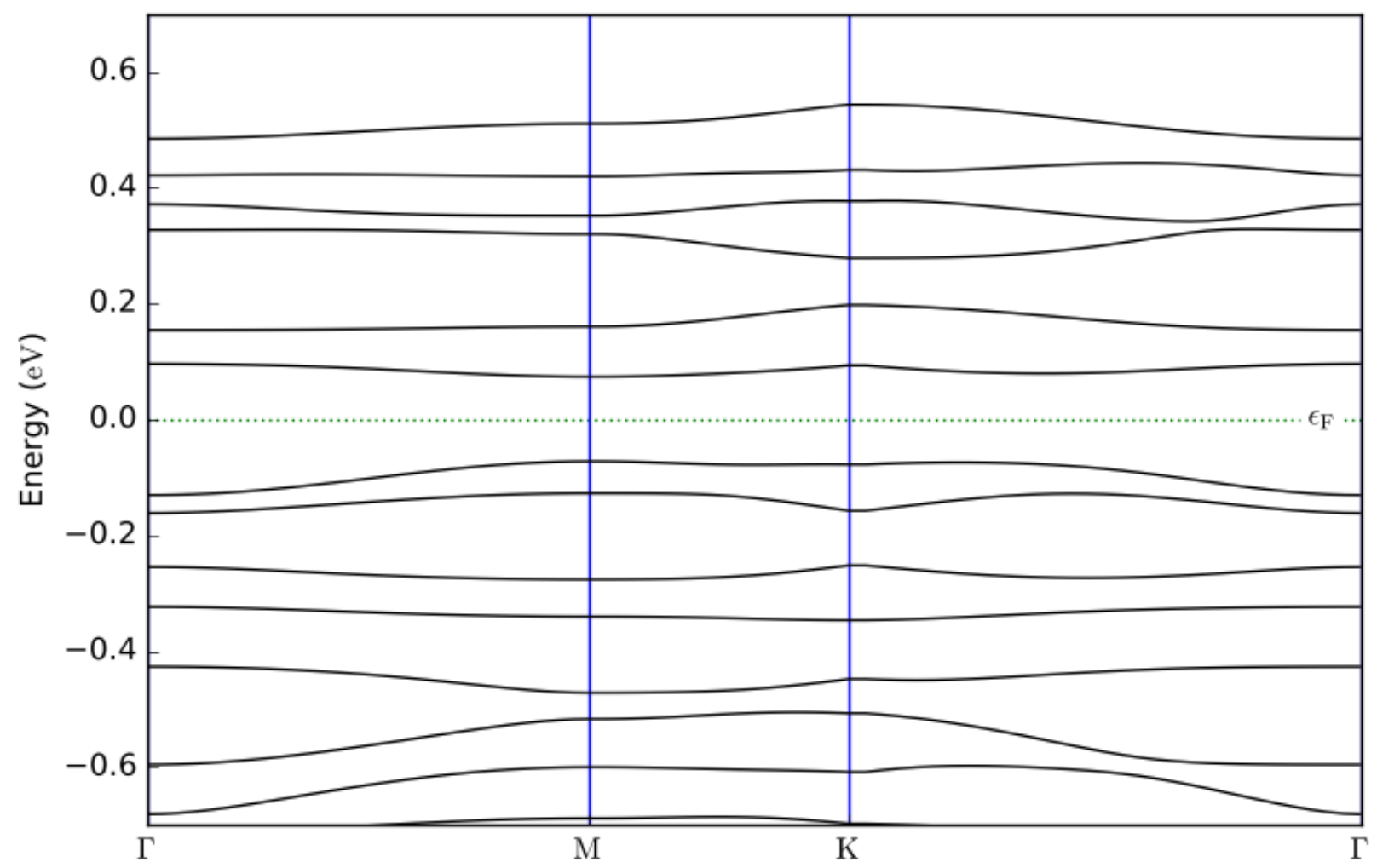

Figure S73: Band structure of $\mathrm{TiCl}_{2} \sqrt{13} \times \sqrt{13} \phi_{-1}$. 


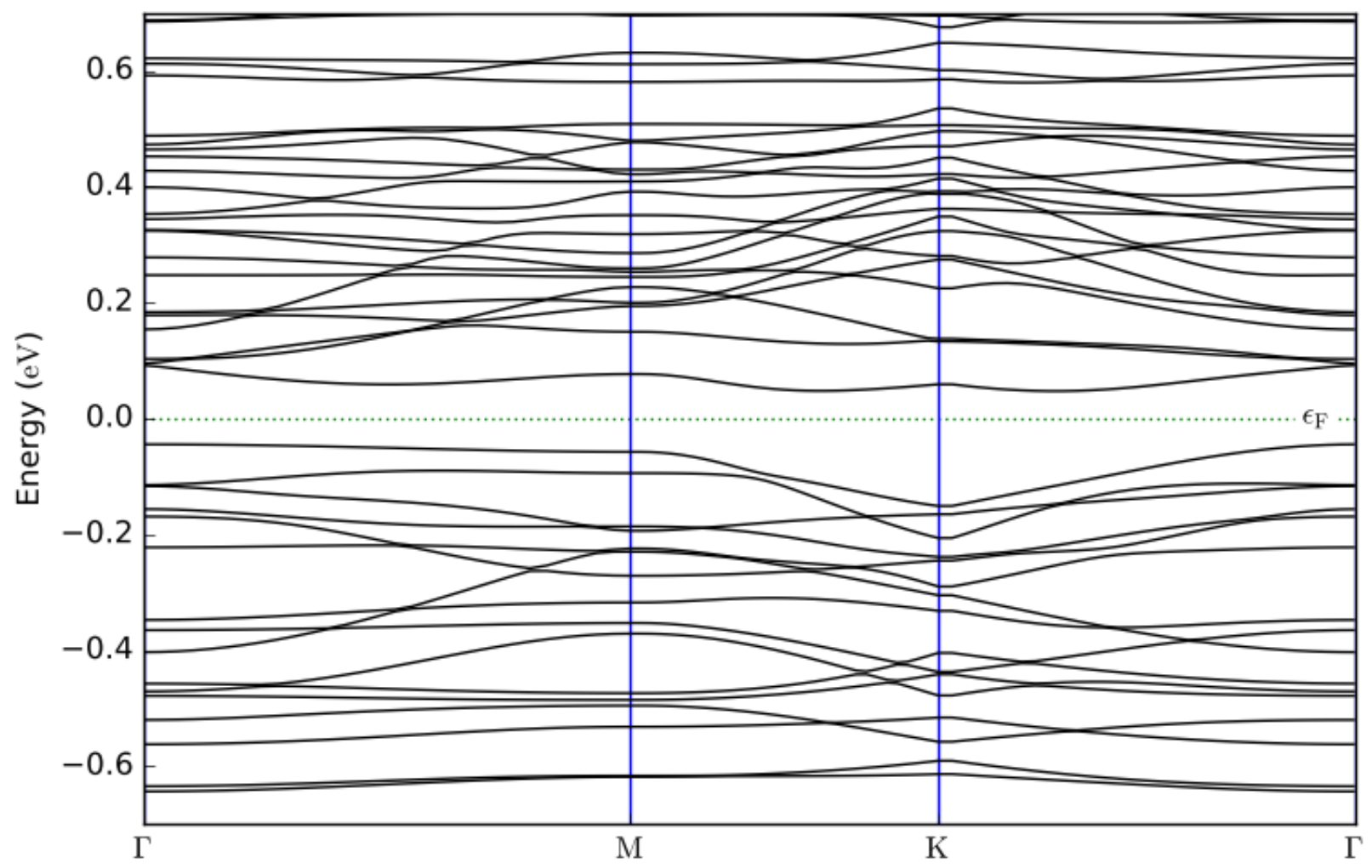

Figure S74: Band structure of TiOCl $4 \times 4 \phi_{-1}$. 


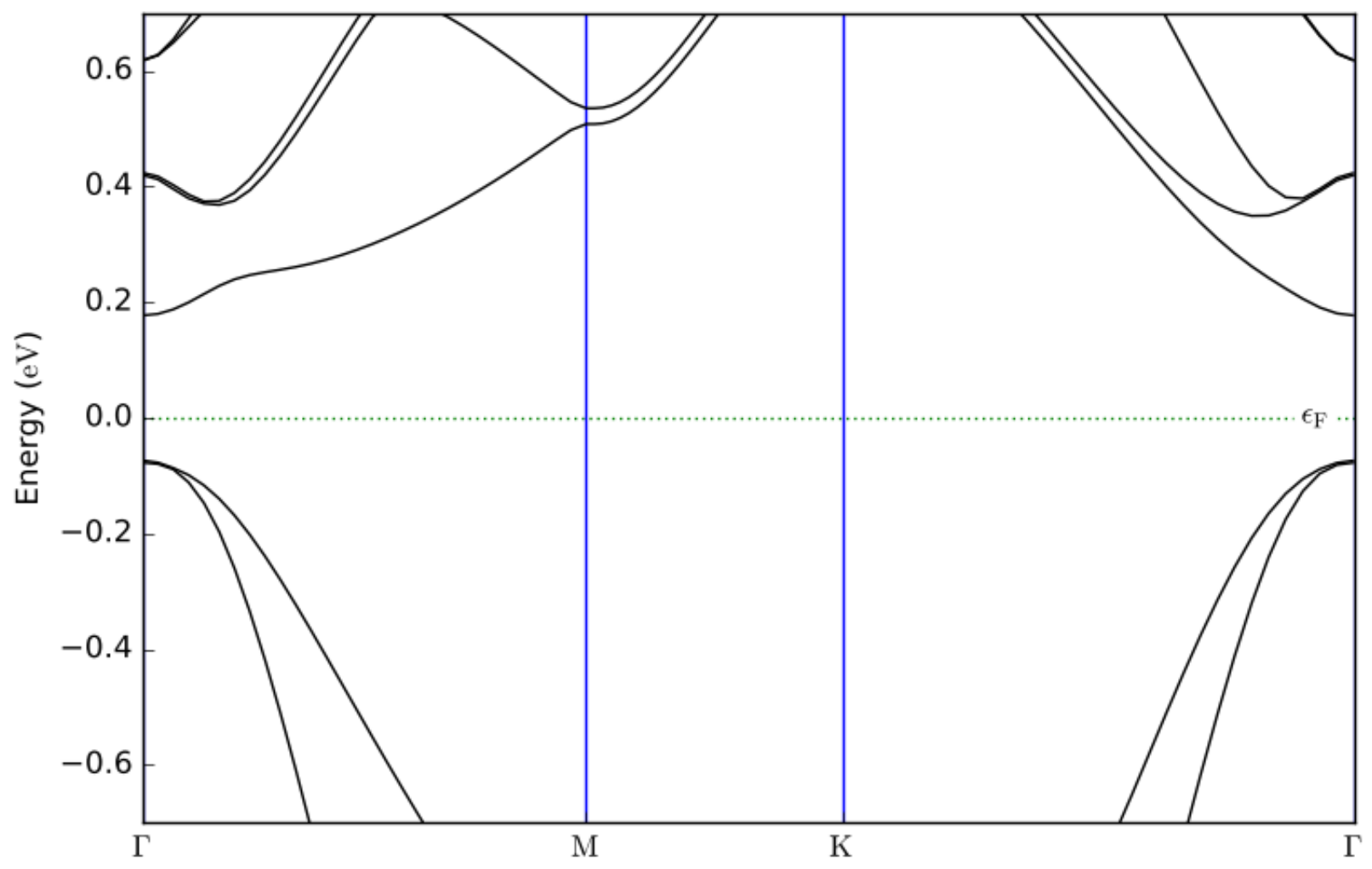

Figure S75: Band structure of $\mathrm{TiS}_{2} 2 \times 2 \phi_{-1}$. 


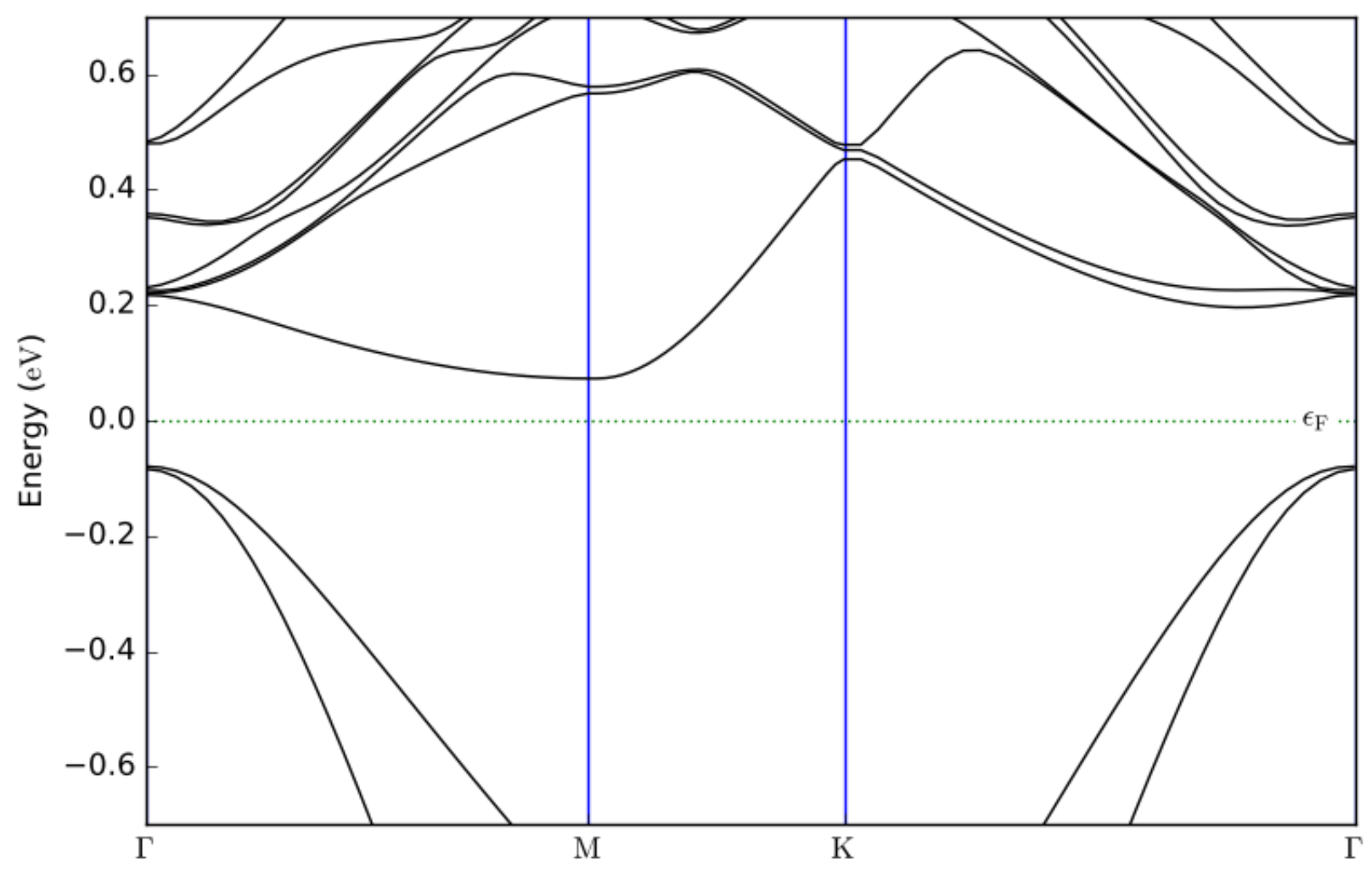

Figure S76: Band structure of $\mathrm{TiS}_{2} 3 \times 3 \phi_{-1}$. 


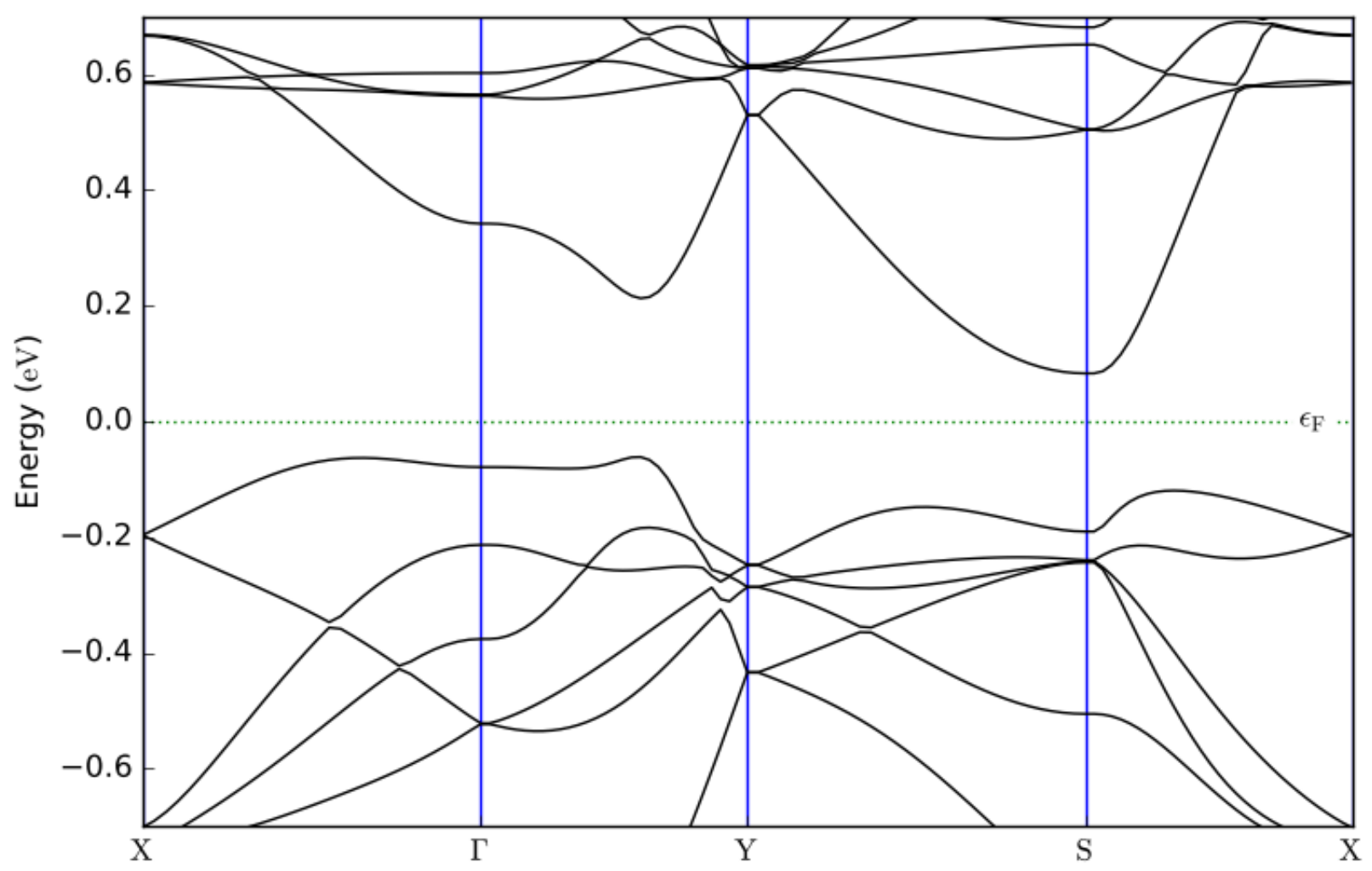

Figure S77: Band structure of VOCl $2 \times 2 \phi_{-2}$. 


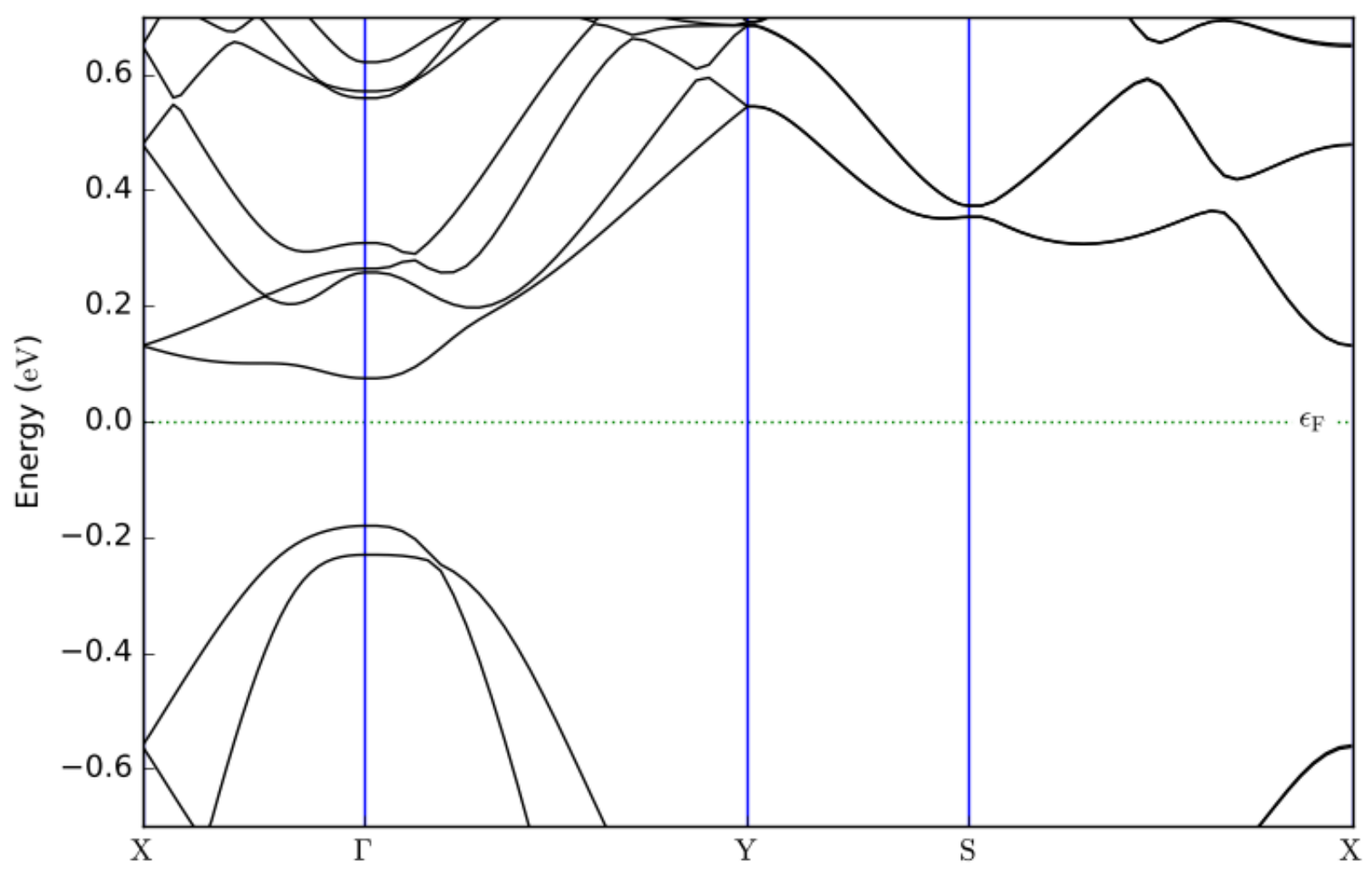

Figure S78: Band structure of $\mathrm{ZrTiSe}_{4} 2 \times 2 \phi_{-1}$. 


\section{Computational Methods}

DFT calculations are carried out using the generalized gradient approximation(GGA) as implemented in the code VASP ${ }^{1-4}$ with the $\mathrm{PAW}^{5}$ method using the Perdew-Burke-Ernzenhof $(\mathrm{PBE})^{6}$ exchange-correlation functional. The pseudopotentials recommended by VASP have been used throughout. Sufficiently large cutoff energy of $550 \mathrm{eV}$ is used to avoid any Pulay stress. For all structural relaxations, a $>\frac{30}{a} \times \frac{30}{b} \times 1$ Gamma-centered $k$-points grid is used to sample the Brillouin zone, where $a$ and $b$ are the lattice parameters of the particular supercell. A $>\frac{60}{a} \times \frac{60}{b} \times 1$ similar $k$-mesh is used for all static runs. The charge densities generated by these static runs have been used to perform the non-self-consistent band structure runs. Electronic convergence is set to be attained when the difference in energy of successive electronic steps becomes less than $10^{-6} \mathrm{eV}$, whereas the structural geometry is optimized until the maximum Hellmann-Feynman force on every atom falls below $0.01 \mathrm{eV} / \AA$. A large vacuum space of $\geq 25 \AA$ in the direction of $\mathbf{c}$ is applied to avoid any spurious interaction between periodically repeated layers.

For the $\mathrm{ZrTiSe}_{4}$ Fermi surface calculation, the code QuantumATK ${ }^{7}$ has been used in conjunction with the PBE functional, where the accuracy of the calculations mostly depends on the selection of norm-conserving pseudopotentials and numerical LCAO basis sets. The "SG15" normconserving pseudopotentials with "Medium" basis sets have been used. The optimized "SG15" provides smooth pseudopotentials with multiple projectors and non-linear core corrections ${ }^{8,9}$. A $7 \times 13 \times 1 k$-points sampling and cutoff energy of 75 Hartree has been employed for both structural relaxation, and band structure/Fermi-surface calculation. The obtained relaxed crystal and band structure is found to be almost identical to those produced by VASP, establishing transferability of the results across different DFT codes and methods.

Phonon calculations are done using the code Phonopy ${ }^{10}$, which uses the atomic forces calculated by VASP based on finite displacement method applied to $2 \times 2$ magnified cells employing a > $\frac{60}{a} \times \frac{60}{b} \times 1 k$-mesh and $10^{-8} \mathrm{eV}$ electronic convergence criteria.

The "Shake \& Relax" protocol of the package AIRSS ${ }^{11}$ interfaced with VASP has been used to produce the distorted structures. A minimum separation of $2 \AA$ is kept between all atoms to minimize unphysical atomic overlaps. The same rigorous DFT relaxation criteria mentioned before 
have been implemented here. The AIRSS Shake \& Relax algorithm and its highly parallel masterslave implementation are similar to that of our previous works ${ }^{12,13}$. To accelerate the DFT calculations, hardware-accelerators have been employed extensively. GPU version of VASP code $^{14,15}$ is used, and regular CPU code is adapted for execution in Xeon-Phi (KNL) ${ }^{16}$.

To generate inputs, parse outputs and measure the RMS displacements, which have been taken as the measure of distortion, the package pymatgen ${ }^{17}$ is employed. The "Mean-shift" algorithm as implemented in the package scikit-learn ${ }^{18}$ is used to perform the unsupervised clustering. The bandwidth for the clustering process has been obtained with the "estimate_bandwidth" function which takes two inputs, the data-set and a quantile value (0.3-0.45). The band structure unfolding has been performed with the tool vaspkit ${ }^{19}$ interfaced with VASP. All crystal-structure images are generated using the tool VESTA ${ }^{20}$.

All CDW crystal structure files (cif) and their top-view images (png) are available at the OSF repository. (https://osf.io/by7et).

\section{Supporting References}

(1) Kresse, G.; Hafner, J. Ab Initio Molecular Dynamics for Liquid Metals. Phys. Rev. B 1993, $47(1), 558-561$.

(2) Kresse, G.; Hafner, J. Ab Initio Molecular-Dynamics Simulation of the Liquid-Metal-Amorphous-Semiconductor Transition in Germanium. Phys. Rev. B 1994, 49 (20), 1425114269.

(3) Kresse, G.; Furthmüller, J. Efficient Iterative Schemes for Ab Initio Total-Energy Calculations Using a Plane-Wave Basis Set. Phys. Rev. B 1996, 54 (16), 11169-11186.

(4) Kresse, G.; Furthmüller, J. Efficiency of Ab-Initio Total Energy Calculations for Metals and Semiconductors Using a Plane-Wave Basis Set. Comput. Mater. Sci. 1996, 6 (1), 1550.

(5) Kresse, G.; Joubert, D. From Ultrasoft Pseudopotentials to the Projector AugmentedWave Method. Phys. Rev. B 1999, 59 (3), 1758-1775. 
(6) Perdew, J. P.; Burke, K.; Ernzerhof, M. Generalized Gradient Approximation Made Simple. Phys. Rev. Lett. 1996, 77 (18), 3865-3868.

(7) QuantumATK with Virtual NanoLab, Synopsys Denmark, https://www.synopsys.com/silicon/quantumatk.html

(8) Hamann, D. R. Optimized Norm-Conserving Vanderbilt Pseudopotentials. Phys. Rev. B Condens. Matter Mater. Phys. 2013, 88 (8), 085117.

(9) Schlipf, M.; Gygi, F. Optimization Algorithm for the Generation of ONCV Pseudopotentials. Comput. Phys. Commun. 2015, 196, 36-44.

(10) Togo, A.; Tanaka, I. First Principles Phonon Calculations in Materials Science. Scr. Mater. 2015, 108, 1-5.

(11) Needs, C. J. P. and R. J. Ab Initio Random Structure Searching. J. Phys. Condens. Matter 2011, 23 (5), 53201.

(12) Kabiraj, A.; Mahapatra, S. High-Throughput First-Principles-Calculations Based Estimation of Lithium Ion Storage in Monolayer Rhenium Disulfide. Commun. Chem. 2018, 1 (1), 81 .

(13) Kabiraj, A.; Mahapatra, S. Intercalation-Driven Reversible Switching of 2D Magnetism. $J$. Phys. Chem. C 2020, 124 (1), 1146-1157.

(14) Hacene, M.; Anciaux-Sedrakian, A.; Rozanska, X.; Klahr, D.; Guignon, T.; FleuratLessard, P. Accelerating VASP Electronic Structure Calculations Using Graphic Processing Units. J. Comput. Chem. 2012, 33 (32), 2581-2589.

(15) Hutchinson, M.; Widom, M. VASP on a GPU: Application to Exact-Exchange Calculations of the Stability of Elemental Boron. Comput. Phys. Commun. 2012, 183 (7), $1422-1426$.

(16) Zhao, Z.; Marsman, M.; Wende, F.; Kim, J. Performance of Hybrid MPI / OpenMP VASP on Cray XC 40 Based on Intel Knights Landing Many Integrated Core Architecture. CUG Conference proceedings 2015.

(17) Ong, S. P.; Richards, W. D.; Jain, A.; Hautier, G.; Kocher, M.; Cholia, S.; Gunter, D.; 
Chevrier, V. L.; Persson, K. A.; Ceder, G. Python Materials Genomics (Pymatgen): A Robust, Open-Source Python Library for Materials Analysis. Comput. Mater. Sci. 2013, $68,314-319$.

(18) Pedregosa, F.; Varoquaux, G.; Gramfort, A.; Michel, V.; Thirion, B.; Grisel, O.; Blondel, M.; Prettenhofer, P.; Weiss, R.; Dubourg, V.; Vanderplas, J.; Passos, A.; Cournapeau, D.; Brucher, M.; Perrot, M.; Duchesnay, E. Scikit-Learn: Machine Learning in Python. $J$. Mach. Learn. Res. 2011, 12, 2825-2830.

(19) Wang, V.; Xu, N.; Liu, J. C.; Tang, G.; Geng, W.-T. VASPKIT: A Pre- and PostProcessing Program for VASP Code. arXiv:1908.08269 2019.

(20) Momma, K.; Izumi, F. VESTA3 for Three-Dimensional Visualization of Crystal, Volumetric and Morphology Data. J. Appl. Crystallogr. 2011, 44 (6), 1272-1276. 